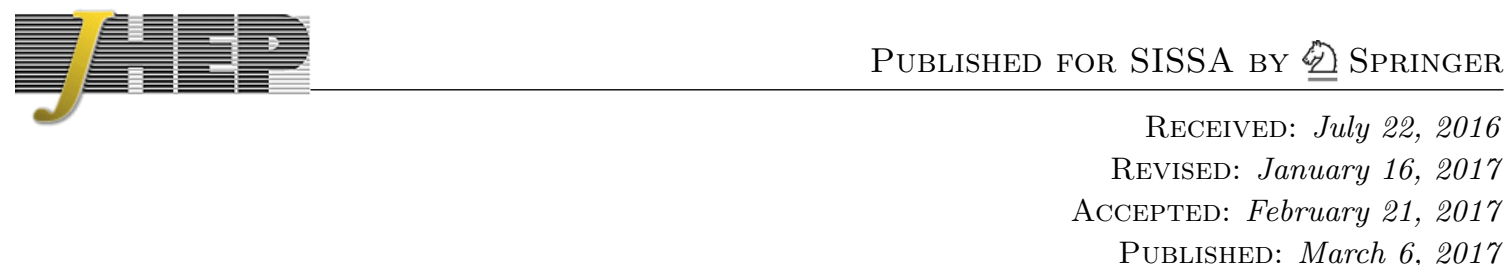

\title{
Higgs mass from neutrino-messenger mixing
}

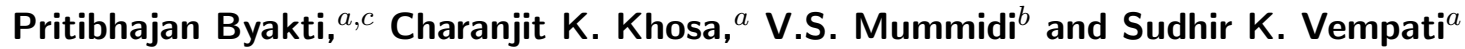 \\ ${ }^{a}$ Center for High Energy Physics, Indian Institute of Science, \\ C.V. Raman Ave, Bangalore 560012, India \\ ${ }^{b}$ Harish-Chandra Research Institute, \\ Chhatnag Road, Jhusi, Allahabad 211019, India \\ ${ }^{c}$ Department of Theoretical Physics, Indian Association for the Cultivation of Science, \\ $2 A$ E3 $2 B$ Raja S.C. Mullick Road, Kolkata 700 032, India \\ E-mail: tppb@iacs.res.in, khosacharanjit@chep.iisc.ernet.in, \\ venkatasuryanarayana@hri.res.in, vempati@chep.iisc.ernet.in
}

ABSTRACT: The discovery of the Higgs particle at $125 \mathrm{GeV}$ has put strong constraints on minimal messenger models of gauge mediation, pushing the stop masses into the multi-TeV regime. Extensions of these models with matter-messenger mixing terms have been proposed to generate a large trilinear parameter, $A_{t}$, relaxing these constraints. The detailed survey of these models $[1,2]$ so far considered messenger mixings with only MSSM superfields. In the present work, we extend the survey to MSSM with inverse-seesaw mechanism. The neutrino-sneutrino corrections to the Higgs mass in the inverse seesaw model are not significant in the minimal gauge mediation model, unless one considers messenger-matter interaction terms. We classify all possible models with messenger-matter interactions and perform thorough numerical analysis to find out the promising models. We found that out of the 17 possible models 9 of them can lead to Higgs mass within the observed value without raising the sfermion masses significantly. The successful models have stop masses $\sim 1.5 \mathrm{TeV}$ with small or negligible mixing and yet a light CP even Higgs at $125 \mathrm{GeV}$.

KEYWORDS: Supersymmetry Phenomenology

ARXIV EPRINT: 1607.03447 


\section{Contents}

1 Introduction 1

2 Recap of GMSB with and without matter-messenger mixing terms $\quad 3$ 2.1 Matter-messenger interactions 4

3 Messenger-matter interactions involving leptons and neutrinos $\quad 6$

$\begin{array}{lll}3.1 & \text { Inverse seesaw model } & 6\end{array}$

3.2 Classification of the models 9

4 Analysis of the models $\quad 11$

4.1 Model $1 \quad 12$

$\begin{array}{lll}4.2 & \text { Model } 2 & 14\end{array}$

$\begin{array}{llr}4.3 & \text { Model } 3 & 16\end{array}$

$\begin{array}{llr}4.4 & \text { Model } 4 & 18\end{array}$

$\begin{array}{lll}4.5 & \text { Model } 5 & 20\end{array}$

4.6 Model $6 \quad 22$

$\begin{array}{lll}4.7 & \text { Model } 7 & 24\end{array}$

$\begin{array}{lll}4.8 & \text { Model } 8 & 26\end{array}$

4.9 Model $9 \quad 27$

$\begin{array}{ll}4.10 \text { Model } 10 & 29\end{array}$

4.11 Model $11 \quad 30$

4.12 Model 12

$\begin{array}{ll}4.13 \text { Model } 13 & 35\end{array}$

$\begin{array}{ll}4.14 \text { Model } 14 & 37\end{array}$

$\begin{array}{ll}4.15 \text { Model } 15 & 39\end{array}$

4.16 Model $16 \quad 41$

4.17 Model $17 \quad 43$

5 Discussion and conclusions $\quad 45$

A One loop neutrino-sneutrino corrections to the Higgs mass 48

\section{Introduction}

Supersymmetry (SUSY) [3-7] offers one of the most elegant solutions to the hierarchy problem. In the Minimal Supersymmetric Standard Model (MSSM) [7-9], the Higgs mass is protected from the dangerous UV sensitive radiative corrections. However, for various reasons, supersymmetry breaking cannot be incorporated in the MSSM in a straightforward 
way. SUSY is spontaneously broken in a remote sector and the information is then passed to the MSSM sector through mediators. Among the different types of mediation mechanisms, gauge mediation (GMSB in short, for a review see [10]) is interesting as it generates soft parameters which are flavor diagonal.

The discovery of $\sim 125 \mathrm{GeV}$ CP even neutral Higgs boson $[11,12]$ has however imposed strong constraints on GMSB models. To accommodate the light CP-even Higgs boson of that mass range, the spectrum of GMSB models had to become heavy [13]. Such a heavy spectrum is not 'natural' as it leads to larger fine tuning. Secondly, there is a bleak chance to discover any such particle at Large Hadron Collider (LHC). This is true for all the GMSB models which are characterized by small A-terms, including the most general one of general gauge mediation (GGM) [14, 15].

Several solutions have been put forward to remedy this situation. They can mainly be divided into two classes: (a) models which generate large A-terms through some mechanism [1, 2, 16-31] (b) models which augment to the Higgs mass through additional contributions while keeping the A-terms small. The former class is dominated by models which contain new interactions between messenger and matter fields. These generate the required A-terms for the stop sector, though some of them could suffer from other problems like $A_{t} / m^{2}$ problem [19]. In the second category several strategies are proposed, for example, $\mathrm{U}(1)$ gauge group extension [32], NMSSM and/or vector matter [19, 33-46], SO(10) D terms [47] to name a few. Another way is to have an additional source of supersymmetry breaking, preferably mediated by gravitational interactions, such that it dominantly generates $A_{t}$ and other related soft terms [48]. In the present work we will focus on the first class of models with messenger-matter interactions. A classification of all such models has been presented in refs. [1, 2]) for MSSM. The classification in [2] concentrated on the messenger interactions with hadronic matter fields $Q, U^{c}$ and the Higgs field $H_{u}$ which are relevant for the $A_{t}$ and other trilinear parameters. In ref. [1], messenger and matter fields interact $\mathrm{SU}(5)$ multiplet-wise. As a consequence other fields like $D^{c}, L, E^{c}$ and $H_{d}$ also interact with the messengers in the studied models. In MSSM, the messenger matter interactions involving leptonic fields will not play any role in the generation of $A_{t}$ or on the Higgs mass. However, the situation changes in the presence of an 'inverse' seesaw mechanism [49].

The standard seesaw mechanism with right handed neutrinos can have large Yukawa couplings $\sim \mathcal{O}\left(y_{t}\right)$, the corrections to the Higgs mass are tiny as the right handed neutrinos are very heavy, close to the GUT scale to give the correct neutrino masses (see ref. [50] and references there in). On the other hand, inverse seesaw mechanism has additional singlets by which the right handed neutrino masses need not be very heavy and this enables corrections to the Higgs mass $[49,51,52]$ which can be significant in some regions of the parameter space. There is however a caveat: the neutrino-sneutrino radiative corrections to the Higgs mass are different compared to the top-stop corrections. In the limit of large right handed neutrino masses, $m_{R} \gg m_{\tilde{\nu}}$ the neutrino and the sneutrino corrections to the Higgs mass cancel each other leading to negligible enhancement to the Higgs mass [51, 52]. However, there are two situations when the corrections to the Higgs mass can be significant: (a) if the slepton and sneutrino masses are comparable to $\sim m_{R}$, typically in the multi-TeV regime and (b) the trilinear parameter associated with the neutrino Yukawa, $X_{N}$ is large, 
leading to large mixing in the sneutrino sector. For case: (a) large slepton masses are not natural in minimal versions of GMSB. One possibility is to consider GGM boundary conditions with a separate and large slepton masses as boundary conditions [52]. For case:(b), trilinear parameters are generally small in minimal messenger models of gauge mediation. To generate large trilinear sneutrino mixing parameter, we consider mattermessenger mixing in the present paper.

As mentioned earlier, we extended the classification of the messenger-matter interaction models to the lepton and neutrino fields. We found that there are 17 models which are tabulated in table 1. Considering GMSB boundary conditions along with these neutrinomessenger couplings we show that light stops can give Higgs mass $\sim 125 \mathrm{GeV}$ in nine of these models. In these mixing models, only the third generation is allowed to couple with messengers. Hence we are safe from flavor constraints.

The paper organizes as follows. In section 2 we summarize gauge mediated SUSY breaking with and without mixing. Then we discuss inability of inverse seesaw models to produce correct Higgs mass in section 3 and motivate the study of messenger-matter interaction involving leptons and right handed neutrino fields. We classify the models based on the messenger-matter interactions. In section 4 classified models are analyzed in detail. Finally in section 5 we conclude.

\section{Recap of GMSB with and without matter-messenger mixing terms}

Gauge mediated SUSY breaking models consist of three sectors: (a) visible sector, (b) messenger sector, and (c) hidden sector. We do not know much about the hidden sector. However it is assumed that SUSY is spontaneously broken there and information of SUSY breaking is encoded in the spurion field $X$. Vacuum expectation value (VEV) of this spurion field is: $\langle X\rangle=M+\theta^{2} F$ where $M$ is the messenger scale and $F$ is SUSY breaking VEV. The spurion field has superpotential level interaction with the messenger fields $\Phi_{m}$ as follows:

$$
W_{\mathrm{mes}}=f X \sum_{i} \bar{\Phi}_{i m} \Phi_{i m}
$$

where superfield $\bar{\Phi}_{m}$ is conjugate representation of $\Phi_{m}$ under SM gauge group. In principle one can have complicated version of the above model; however, it is the simplest one and is called minimal GMSB (mGMSB) model. In general, messenger fields are multiplets of $\mathrm{SU}(5)$ like 5, 10 and 15 dimensional representations. Messenger fields are not, in general, considered to be incomplete multiplets of $\mathrm{SU}(5)$ as it may destroy one of beautiful features of MSSM, which is the unification of gauge coupling constants. However one can use incomplete multiplets as messengers without spoiling unification in special cases [53, 54].

Because of non-zero F-term VEV of $X$, messenger sector is not supersymmetric. As the messenger fields are charged under gauge groups, the SUSY breaking information passes to the visible sector through gauge interactions. Gaugino masses are generated at 1-loop level:

$$
M_{r}=\frac{\alpha_{r}}{4 \pi} d N \Lambda g(x)
$$


where $r=1,2,3$ represents $\mathrm{U}(1), \mathrm{SU}(2)$ and $\mathrm{SU}(3)$ groups respectively, $\alpha_{r}=g_{r}^{2} / 4 \pi$, $\Lambda=F / M, x=F / M^{2}, \mathrm{~d}$ is the Dynkin index, $N$ is the number of messengers and the function $g(x)$ has the following form:

$$
g(x)=\frac{1}{x^{2}}[(1+x) \ln (1+x)]+(x \rightarrow-x) .
$$

Scalar soft mass squares are generated at 2-loop level,

$$
M_{\tilde{a} \text { usual }}^{2}=2 N d \Lambda^{2}\left[\sum_{r} C_{r}(a)\left(\frac{\alpha_{r}}{4 \pi}\right)^{2}\right] f(x),
$$

where $C_{r}(a)$ is the quadratic Casimir of the representation of the MSSM field labeled by ' $a$ ' and the group corresponding to $r, \tilde{a}$ is the super-partner of the field $a$, and the function $f(x)$ has the following form:

$$
f(x)=\frac{1+x}{x^{2}}\left[\ln (1+x)-2 L i_{2}\left(\frac{x}{1+x}\right)+\frac{1}{2} L i_{2}\left(\frac{2 x}{1+x}\right)\right]+(x \rightarrow-x) .
$$

Note that gaugino masses are proportional to $N$ whereas the sfermion masses are proportional to $\sqrt{N}$. Thus one can have heavier gauginos with fixed sfermion masses for a larger $N$. Same is also true for the Dynkin index $d$. One gets heavier gauginos with fixed sfermion masses for a $10 \oplus \overline{10}$ messenger as compared to a copy of $5 \oplus \overline{5}$ messenger field.

The spectrum changes in accordance with the messenger sector. In refs. [55, 56] expressions for soft masses were derived without considering any model for the messenger and the hidden sector. This model, as it encompasses all the GMSB models, is known as general gauge mediation or GGM. Expressions for the soft masses are as follows:

$$
\begin{aligned}
M_{r} & =\alpha_{r} B_{r}, \\
M_{\tilde{a} \text { usual }}^{2} & =\sum_{r} \alpha_{r}^{2} C_{r}(a) A_{r} .
\end{aligned}
$$

Now we see that instead of one scale $\Lambda$ there are six dimensionful parameters, $B_{r}$ and $A_{r}$. In principle they can be arbitrary. GGM thus predicts non-universal gauginos without spoiling the gauge coupling unification.

As A-terms are not generated even in GGM, none of the pure GMSB models can explain the Higgs mass with a light stop spectrum. In order to explain the Higgs mass one either requires stop masses $\gtrsim 4 \mathrm{TeV}$ or maximum mixing in the stop sector [13]. One way of generating mixing term or A-term at the boundary is to consider messenger-matter interactions $[18,57]$.

\subsection{Matter-messenger interactions}

The idea of extending GMSB models by considering messenger-matter interactions is not new $[18,57]$. In particular, to solve the severe $\mu-B_{\mu}$ problem in GMSB, one needs to couple the Higgs sector with the messenger sector [58-60]. In ref. [61], contribution of the messenger-matter interactions to other soft masses and the A-terms was calculated using wavefunction renormalization technique [62]. After the discovery of the Higgs particle this 
idea became popular as it can save GMSB models by generating large stop mixing parameter. Several works were presented in this idea [16, 17, 19-21]. In refs. [1, 2], messengermatter interactions were classified and thoroughly studied in MSSM. In [1] messengers are assumed to be 1, 5 and 10 dimensional representations of $\mathrm{SU}(5)$ and these messengers are interacting with the MSSM SU(5) multiplet-wise. In principle messenger-matter interactions can introduce flavor violation. Peccei-Quinn symmetry was used to suppress the flavor violation as well as to classify the models. The general classification reproduced older models [16-21] and as well as found some new models. On the other hand in ref. [2] messengers are allowed to interact with MSSM fields by SM multiplet-wise. More recent works in this direction can be found in the refs. [22-31, 47, 63]. Models with explicit flavour violation can be found in $[31,64]$.

Messenger-matter interactions are classified into two types depending on the number of matter fields in the interaction: (a) Type I where one matter field interacts with two messenger fields, and (b) Type II where one messenger field interacts with two matter fields. Superpotentials for these two types are given as:

$$
W_{\text {mix }}=\left\{\begin{array}{ll}
\frac{1}{2} \lambda_{a A B} \Phi_{a} \Phi_{A m} \Phi_{B m} & \text { Type I } \\
\frac{1}{2} \lambda_{a b A} \Phi_{a} \Phi_{b} \Phi_{A m} & \text { Type II }
\end{array},\right.
$$

where $a, b, c \cdots$ is used to indicate visible sector fields and the capital indices $A, B, C, \cdots$ are used to indicate messenger fields. Because of presence of these couplings, one gets 1-loop correction to the soft scalar mass-squared as follows

$$
\delta_{\text {1-loop }} M_{\tilde{a}}^{2}=-\frac{x^{2} \Lambda^{2} h(x)}{96 \pi^{2}}\left\{\begin{array}{l}
\sum_{B C} d_{a}^{B C}\left|\lambda_{a B C}\right|^{2} \text { Type I, } \\
\sum_{b B} d_{a}^{b B}\left|\lambda_{a b B}\right|^{2} \text { Type II, }
\end{array}\right.
$$

$d_{\text {indices }}$ is a group theoretical factor which appears in beta functions and $h(x)$ has the following form:

$$
h(x)=3 \frac{(x-2) \ln (1-x)}{x^{4}}+(x \rightarrow-x) .
$$

Note that 1-loop correction is always negative and it contributes only to the fields which are directly coupled to the messenger fields. Another point is that these contribution are suppressed for small values of $x \lesssim 0.1$ and dominant for $x \sim 0.5$. The A-terms and 2-loop corrections are usually calculated using wavefunction renormalization technique $[2,61,62]$. For Type I and Type II models these corrections are as follows [2]:

\section{Type I models.}

$$
\begin{aligned}
& A_{a}=-\frac{1}{8 \pi} \sum_{B, C} d_{a}^{B C}\left|\alpha_{a B C}\right| \Lambda \\
& \delta_{2-\mathrm{loop} M_{\tilde{a}}^{2}=\frac{1}{16 \pi^{2}}}\left[\sum_{B, C, D, c} d_{a}^{B C} d_{B}^{c D}\left|\alpha_{a B C}\right|\left|\alpha_{c B D}\right|+\frac{1}{4} \sum_{B, C, D, E} d_{a}^{B C} d_{a}^{D E}\left|\alpha_{a B C}\right|\left|\alpha_{a D E}\right|\right. \\
&\left.-\frac{1}{2} \sum_{B, C, c, d} d_{a}^{c d} d_{c}^{B C}\left|\alpha_{a c d}\right|\left|\alpha_{c B C}\right|-\sum_{B, C} d_{a}^{B C} C_{r}^{a B C} \alpha_{r}\left|\alpha_{a B C}\right|\right] \Lambda^{2},
\end{aligned}
$$

where we used $\alpha_{\text {indices }}$ to denote $\lambda_{\text {indices }}^{2} / 4 \pi$. 


\section{Type II models.}

$$
\begin{aligned}
A_{a}= & -\frac{1}{4 \pi} \sum_{B, c} d_{a}^{c B}\left|\alpha_{a c B}\right| \Lambda \\
\delta_{2-\text { loop } M_{\tilde{a}}^{2}=} & \frac{1}{16 \pi^{2}}\left[\frac{1}{2} \sum_{B, c, d, e} d_{a}^{c B} d_{B}^{d e}\left|\alpha_{a c B}\right|\left|\alpha_{d e B}\right|+\sum_{B, C, c, d} d_{a}^{c B} d_{c}^{d C}\left|\alpha_{a c B}\right|\left|\alpha_{c d C}\right|\right. \\
& +\sum_{B, C, c, d} d_{a}^{c B} d_{a}^{d C}\left|\alpha_{a c B}\right|\left|\alpha_{a d C}\right|-\sum_{B, c, d, f} d_{a}^{c d} d_{c}^{f B}\left|\alpha_{a c d}\right|\left|\alpha_{c f B}\right| \\
& +\frac{1}{32 \pi^{2}} \sum_{B, c, d, e, f} d_{a}^{c d} d_{c}^{e f} y_{a c d}^{*} y_{c e f} \lambda_{a d B} \lambda_{e f B}^{*}+\frac{1}{32 \pi^{2}} \sum_{B, c, d, e, f} d_{a}^{c B} d_{B}^{e f} \lambda_{a c B}^{*} \lambda_{e f B} y_{a c d} y_{d e f}^{*} \\
& \left.-2 \sum_{B, c} d_{a}^{c B}\left(C_{r}^{a}+C_{r}^{c}+C_{r}^{B}\right) \alpha_{r}\left|\alpha_{a c B}\right|+\frac{1}{2} \sum_{B, c, e, f} d_{a}^{c B} d_{c}^{e f}\left|\alpha_{c e f}\right|\left|\alpha_{a c B}\right|\right] \Lambda^{2} .
\end{aligned}
$$

Thus the total soft masses at the boundary are

$$
M_{\tilde{a}}^{2}=M_{\tilde{a} \text { usual }}^{2}+\delta_{1-\text { loop }} M_{\tilde{a}}^{2}+\delta_{2-\text { loop }} M_{\tilde{a}}^{2} .
$$

\section{Messenger-matter interactions involving leptons and neutrinos}

Before studying the messenger-matter mixing terms involving leptonic fields and right handed neutrinos, we are going to review the inverse seesaw model.

\subsection{Inverse seesaw model}

The canonical seesaw mechanism requires extension of the MSSM with a heavy field which could be right handed neutrino or triplet Higgs or a triplet fermion (for a review see [65]). The smallness of the neutrino mass is associated with the heaviness of the additional particle. In the canonical seesaw mechanism, the corrections to the Higgs mass are typically very tiny as the right handed neutrino scale is very heavy $\geq 10^{14} \mathrm{GeV}$. Presence of matter messenger mixing terms will not improve the situation. Note that the right handed neutrino $\left(N^{c}\right)$ mass must be less than the messenger scale, otherwise at the messenger scale, $N^{c}$ fields will be integrated out and messenger-matter interactions involving $N^{c}$ will now reduce to higher dimensional operator at the messenger scale. If we get any trilinear scalar coupling from these operators then they must be suppressed not only by the $N^{c}$ mass but also from loop factors. However, for $N^{c}$ mass up to $10^{5} \mathrm{GeV}$, the allowed value of the Yukawa coupling $\left(y_{N}\right)$ can be utmost $10^{-5}$ to get neutrino mass of $\mathcal{O}(\mathrm{eV})$. Because of such a small value of $y_{N}$, contribution to the Higgs mass from the neutrino sector is negligibly small.

The situation drastically improves in inverse seesaw model [66]. Supersymmetric version of this model has the following superpotential [51, 67]:

$$
\begin{aligned}
& W=U^{c} \mathbf{Y}_{\mathbf{u}} Q H_{u}-D^{c} \mathbf{Y}_{\mathbf{d}} Q H_{d}-E^{c} \mathbf{Y}_{\mathbf{e}} L H_{d}+\mu H_{u} H_{d} \\
& +N^{c} y_{N} L H_{u}+m_{R} N^{c} S+\frac{1}{2} \mu_{s} S^{2},
\end{aligned}
$$

where the MSSM fields are in standard notation with $\mathbf{Y}_{\mathbf{u}}$ etc, representing the Yukawa matrices for three generations and the $N^{c}$ and $S$ are new fields added to the MSSM field 
content. These are singlet scalar superfields. Note that if we set $\mu_{s}$ to zero, the above superpotential enjoys $\mathrm{U}(1)$ lepton number symmetry. Its presence implies that this symmetry is softly broken. As $\mu_{s} \rightarrow 0$ restores the symmetry, it can be (technically) naturally small. In the above superpotential we have considered only one generation (third) for the inverse seesaw sector. The generalisation to three generations is straight forward and has minor impact on our analysis.

In the basis $\left\{\nu_{L}, N^{c}, S\right\}$, the mass matrix $M_{\nu}$ of the neutral leptons for one generation, is given by

$$
M_{\nu}=\left(\begin{array}{ccc}
0 & m_{D} & 0 \\
m_{D} & 0 & m_{R} \\
0 & m_{R} & \mu_{s}
\end{array}\right),
$$

where $m_{D}=y_{N}\left\langle H_{u}\right\rangle$. The eigenvalues of the above mass matrix are as follows:

$$
\begin{aligned}
& m_{\nu_{1}} \approx \frac{m_{D}^{2} \mu_{s}}{m_{R}^{2}}, \\
& m_{\nu_{2}} \approx-\left(\frac{m_{D}^{2}}{2 m_{R}}+m_{R}\right), \\
& m_{\nu_{3}} \approx\left(\frac{m_{D}^{2}}{2 m_{R}}+m_{R}\right) .
\end{aligned}
$$

Here $m_{\nu_{1}}$, the lightest neutrino eigenvalue, is proportional to the parameter $\mu_{s}$. From electroweak precision data [68], $m_{D} \lesssim 0.05 m_{R}$ and thus last two eigenvalues of the mass matrix are degenerate. As $m_{D}$ and $m_{R}$ related, $y_{N}$ and $m_{R}$ are also related:

$$
y_{N}=\sqrt{\frac{m_{\nu_{1}}}{\mu_{s}}} \frac{\sqrt{2}}{v} \operatorname{cosec} \beta m_{R},
$$

where $v$ is the electroweak VEV of the Higgs fields: $v=\sqrt{\left\langle H_{u}\right\rangle^{2}+\left\langle H_{d}\right\rangle^{2}}=246 \mathrm{GeV}$. For a fixed $\mu_{s}$, which we fix it to be electron mass, we see that $y_{N}$ scales as $m_{R}$.

The scalar potential for this model is given below which contains SUSY preserving as well as SUSY breaking soft terms:

$$
V_{S}=V_{F}+V_{D}+V_{\text {soft }}
$$

where

$$
\begin{aligned}
V_{F}= & \left|Y_{e} \tilde{E}^{c} H_{d}+y_{N} H_{u} \tilde{N}\right|^{2}+\left|Y_{u} \tilde{Q} \tilde{U}^{c}+\mu H_{d}+y_{N} \tilde{L} \tilde{N}^{c}\right|^{2}+\left|y_{N} \tilde{L} H_{u}+m_{R} \tilde{S}\right|^{2} \\
& +\left|m_{R} \tilde{N}^{c}+\mu_{s} \tilde{S}\right|^{2}+\ldots, \\
V_{D}= & \frac{1}{8}\left(g^{2}+g^{\prime 2}\right)\left(\left|H_{u}\right|^{2}-\left|H_{d}\right|^{2}\right), \\
V_{\text {soft }}= & A_{N} y_{N} \tilde{L} H_{u} \tilde{N}^{c}+B_{R} \tilde{N} \tilde{S}^{c}+B_{S} \tilde{S}^{\dagger} \tilde{S}+\text { h.c. }+M_{N} \tilde{N}^{c \dagger} \tilde{N}^{c}+\cdots .
\end{aligned}
$$

To calculate the neutrino-sneutrino correction to Higgs mass, one needs to calculate the sneutrino mass matrix which has the form:

$$
\mathcal{M}_{\tilde{\nu}}^{2}=\left(\begin{array}{ccc}
M_{\tilde{L}}^{2}+D_{L}+m_{D}^{2} & m_{D}\left(A_{N}-\mu \cot \beta\right) & m_{R} m_{D} \\
* & m_{D}^{2}+M_{N}^{2}+m_{R}^{2} & B_{R}+m_{R} \mu_{s} \\
* & * & m_{R}^{2}+\mu_{s}^{2}+m_{\tilde{S}}^{2}
\end{array}\right),
$$


where the basis is $\left\{\tilde{\nu}_{L}, \tilde{N}^{c}, \tilde{S}\right\}, M_{\tilde{L}}^{2}$ is the slepton mass, and $m_{\tilde{S}}$ is the soft mass of $S$. As the mass matrix is symmetric, terms omitted can be easily understood. As the field $N^{c}$ and $S$ are gauge singlets, soft masses $B_{R}, B_{S}$ and $M_{N}$ are zero at the boundary, the messenger scale. Assuming these are small and $m_{D} / m_{R}<1$, one obtains the following eigenvalues [52]:

$$
\begin{aligned}
& m_{\tilde{\nu}_{1}}^{2} \approx M_{\tilde{L}}^{2}+m_{D}^{2}\left(1+\frac{m_{R}^{2}}{d_{2}}+\frac{X_{N}^{2}}{d_{1}}\right), \\
& m_{\tilde{\nu}_{2}}^{2} \approx M_{N}^{2}+m_{R}^{2}+m_{D}^{2}\left(1-\frac{X_{N}^{2}}{d_{1}}\right), \\
& m_{\tilde{\nu}_{3}}^{2} \approx m_{\tilde{S}}^{2}+m_{R}^{2}-\frac{m_{R}^{2} m_{D}^{2}}{d_{2}}
\end{aligned}
$$

where

$$
\begin{aligned}
d_{1} & =M_{\tilde{L}}^{2}-M_{N}^{2}-m_{R}^{2}, \\
d_{2} & =M_{\tilde{L}}^{2}-m_{R}^{2}-m_{\tilde{S}}^{2}, \\
X_{N} & =A_{N}-\mu \cot \beta .
\end{aligned}
$$

To compute the corrections to the Higgs mass, we use the effective potential method [69]. The one-loop effective potential for neutrino-sneutrino sector is [51, 52]:

$$
V_{1-\text { loop }}^{\nu / \tilde{\nu}}\left(Q^{2}\right)=\frac{2}{64 \pi^{2}}\left[\sum_{i=1}^{3} m_{\tilde{\nu}_{i}}^{4}\left(\log \frac{m_{\tilde{\nu}_{i}}^{2}}{Q^{2}}-\frac{3}{2}\right)-\sum_{i=1}^{3} m_{\nu_{i}}^{4}\left(\log \frac{m_{\nu_{i}}^{2}}{Q^{2}}-\frac{3}{2}\right)\right]
$$

where first and second term represent the contribution of sneutrino and neutrino mass eigenstates respectively. An overall factor 2 takes care of the degrees of freedom for the complex scalar and Weyl fermion. The complete calculation of the correction to the Higgs mass is given in the appendix A. It should be noted that the calculation presented in appendix $\mathrm{A}$ is a slight generalisation of the one presented in ref. [52] as we relaxed the assumption that $X_{N}$ is a small parameter.

Without going into details of sneutrino-neutrino sector corrections to the Higgs mass, we can make the following observations:

1. If $m_{R} \gg M_{\mathrm{SUSY}}$, the sneutrino (eq. (3.10)) and neutrino (eq. (3.3)) eigenvalues are degenerate and are of order of $m_{R}$. There will be a complete cancellation between the scalar and fermion sector contributions and consequently no significant correction to the Higgs mass.

2. To have a significant corrections to the Higgs mass, one should have heavy sleptons [52]. In this case the degeneracy between the scalar and fermion eigenvalues breaks and therefore cancellation will not be exact. Since these corrections are inversely proportional to $d_{1,2}$, for heavy sleptons (comparable to $m_{R}$ ), $d_{1,2}$ will be small and consequently one will get significant enhancement to the Higgs mass. Such large sleptons masses can be generated in a framework like general gauge mediation. An alternative way of enhancing the Higgs mass is through matter-messenger corrections 
which can generate a large $X_{N}$ parameter and/or significant corrections to $M_{\tilde{L}}^{2}$. In this case, the sneutrino derivatives are now proportional to $X_{N}$ (see appendix A for explicit expressions), the sneutrino contribution will be more as compared to neutrino contribution.

Both the above conditions (large $X_{N}$ and very heavy sleptons) are not met in the minimal gauge mediation model. Thus the Higgs mass corrections remain small. Clearly both the scenarios with enhanced corrections are not applicable in minimal GMSB. One could however argue to increase the messenger scale, but this would only increase the mass of the stops which is contrary of our philosophy of keeping stops light.

The possibility of increasing the sneutrino/neutrino contributions by increasing the slepton mass in a general gauge mediation model was discussed in ref. [52]. In the present work, we discuss the importance of the combination of heavy sleptons and large $X_{N}$ parameter (generated through matter-messenger mixing).

\subsection{Classification of the models}

We are interested to study the effect of messenger-matter interaction in the inverse seesaw mechanism. We know that the Lagrangian given in eq. (3.1) has softly broken U(1) lepton number and the softly broken parameter, $\mu_{s}$, is responsible for the generation of neutrino mass through inverse seesaw. If messenger-matter interactions do not obey U(1) lepton number then we cannot guarantee that inverse seesaw is the only source of neutrino mass. Therefore we impose U(1) lepton number on the messenger fields. To generate $A_{t}$ and/or $A_{N}$, at least one of the fields $Q, U^{c}, H_{u}, L$ and $N^{c}$ has to couple with the messenger fields. Models involving $N^{c}$ field in the messenger-matter interaction are not explored in the literature. We have listed 17 possible models of messenger-matter interactions involving $L$ and $N^{c}$ fields in table 1 . In these models we allow only the third generation of the matter fields to couple with the messenger fields. The interaction term $L^{2} E_{m}^{c}$ is not there in the above list because this vanishes. Along with the interaction terms listed above, some new terms may be allowed by symmetry. For example, in model 5 , the term $S^{2} E_{m}^{c}$ is allowed. However, we are not considering this term as it will not generate $A_{t}$ or $A_{N}$. For the same reason, we do not list the models involving only $E^{c}$ or $S$. Each of the model contains the shown interaction in the superpotential. Inter-generational mixing is considered to be absent. When more than one messenger fields is considered, the matter-messenger coupling is considered universal over all the messenger fields.

Some of the models involving $L$, like model 11, 13 and 15, are not new. These are considered in ref. [1] along with other interaction terms. As mentioned earlier, the suffix $m$ is used to indicate messengers. Messenger field with known symbol has the same quantum number under SM gauge group. Here models 10 and 17 are Type I models and rest of the models are of Type II. In each model we are allowing only one messenger-matter interaction term. In the next section, we are going to list the modification of the boundary conditions due to these messenger-matter interactions. 


\begin{tabular}{|c|c|c|c|}
\hline Model No & Interaction & Lepton number & Remarks or Source \\
\hline \multicolumn{4}{|c|}{ Models with $N^{c}$} \\
\hline 1. & $N^{c} Q \bar{Q}_{m}$ & 1 & $\in \overline{10}$ \\
\hline 2. & $N^{c} U^{c} \bar{U}_{m}^{c}$ & 1 & $\in \overline{10}$ \\
\hline 3. & $N^{c} D^{c} \bar{D}_{m}^{c}$ & 1 & $\in 5$ \\
\hline 4. & $N^{c} L H_{u}^{m}$ & 0 & $\in 5$ \\
\hline 5. & $N^{c} E^{c} \bar{E}_{m}^{c}$ & 2 & $\in \overline{10}$ \\
\hline 6. & $N^{c} H_{u} H_{d}^{m}$ & 1 & $\in \overline{5}$ \\
\hline 7. & $N^{c} H_{d} H_{u}^{m}$ & 1 & $\in 5$ \\
\hline 8. & $\frac{1}{2}\left(N^{c}\right)^{2} S_{m}$ & 2 & $\in 1$ \\
\hline 9. & $N^{c} S S_{m}$ & 0 & $\in 1$ \\
\hline 10. & $N^{c} H_{u}^{m} H_{d}^{m}$ & --- & $\in 5 \oplus \overline{5}$ \\
\hline \multicolumn{5}{|c|}{ Models with L } \\
\hline 11. & $L Q D_{m}^{c}$ & -1 & $\in \overline{5}$ \\
\hline 12. & $L D^{c} Q_{m}$ & -1 & $\in 10$ \\
\hline 13. & $L E^{c} H_{d}^{m}$ & 0 & $\in \overline{5}$ \\
\hline 14. & $L H_{d} E_{m}^{c}$ & -1 & $\in 10$ \\
\hline 15. & $L H_{u} S_{m}$ & -1 & $\in 1$ \\
\hline 16. & $L S H_{u}^{m}$ & -2 & $\in 5$ \\
\hline 17. & $L H_{u}^{m} S_{m}$ & --- & $\in 1,5$ \\
\hline
\end{tabular}

Table 1. Classification of the models. Note that, for the models 1, 2, 5 and 14, messenger fields are $10 \oplus \overline{10}$ and for the rest of the models these are $5 \oplus \overline{5}$. Each model contains only one term and the corresponding coupling is chosen to be $\lambda$. In the third column lepton number of the messenger fields are listed. As in the model 10 and 17 two messenger fields are appearing in each interaction terms, one can assign any lepton number to the messengers keeping in mind that product of their lepton numbers should be 1 and -1 for the model 10 and 17 respectively. In the last column we mention the representation and source of the messenger field or fields. 


\section{Analysis of the models}

From the previous section it is clear that the fields which are coupled with the messenger fields through Yukawa interactions have negative one-loop corrections as well as positive two-loop corrections to their soft masses. Other matter fields which are not directly coupled to the messenger fields but, have Yukawa interactions with the matter fields with direct interactions to the messenger fields, always get two-loop negative contributions. On the top of these corrections, there are usual GMSB contributions to the soft masses which are always positive. The messenger-matter coupling $\lambda$ cannot take arbitrary values as it can lead to negative mass squared eigenvalues for the scalars at the weak scale.

Another important parameter is $x \equiv \Lambda / M$. One-loop corrections diminish for smaller values of $x$. We consider two cases: (a) $x=0.5$ for which we cannot neglect 1-loop effects, and (b) $x=0.1$ for which 1-loop contributions can be neglected.

Because of non-observation of any SUSY particle, LHC bounds on the soft masses are very stringent. In GGM models, the present lower limits on gluino is $1.6 \mathrm{TeV}$ [70], whereas on the chargino it is $650 \mathrm{GeV}$ [71]. It can be easily seen that LHC bound on chargino mass is more stronger than that of the gluino mass in the universal gaugino mass case. If one considers mGMSB with $N=d=1$ then the upper bound on the gluino mass forces the stop masses to be of the order of $2 \mathrm{TeV}$. However one could be interested in light spectrum for various reasons including the fine-tuning issue. To resolve this issue in the models with $5 \oplus \overline{5}$, we consider the number of messengers to be 3 . In models $1,2,5$ and 14 this problem is automatically solved as $10 \oplus \overline{10}$ messenger field has $d=3$. We choose the following values in the numerical analysis:

\begin{tabular}{|c|c|c|}
\hline & $5 \oplus \overline{5}$ & $10 \oplus \overline{10}$ \\
\hline Models & $3,4,6,7, \cdots, 11,13,15,16,17$ & $1,2,5,12,14$ \\
\hline$\Lambda$ & $100 \mathrm{TeV}$ & $100 \mathrm{TeV}$ \\
\hline Number of messengers & 3 & 1 \\
\hline Dynkin index & 1 & 3 \\
\hline
\end{tabular}

Gravitino is the LSP in these models. Its mass has the following expression:

$$
m_{\frac{3}{2}}=\frac{F}{\sqrt{3} M_{\mathrm{Pl}}}=\frac{\Lambda^{2}}{\sqrt{3} x M_{\mathrm{Pl}}}=\frac{10}{4.16 x} \mathrm{eV},
$$

where $M_{\mathrm{Pl}}=2.4 \times 10^{18} \mathrm{GeV}$. Thus we get gravitino mass $4.8 \mathrm{eV}$ and $24 \mathrm{eV}$ for $x=0.5$ and $x=0.1$, respectively. Experimental bound on gravitino mass at $2 \sigma$ limit is $16 \mathrm{eV}$ [72]. Though $x=0.1$ case is ruled out by gravitino mass constraint, there is a way out to overcome this gravitino problem [73].

Lepton number violating mass parameter $\mu_{s}$ is another important parameter. Upper limit of $y_{N}$ depends on it. The upper bound on $y_{N}$ comes from electroweak precision tests, which sets the ratio $m_{D} / m_{R}<\frac{1}{20}$ [68]. We consider $\mu_{s}=5 \times 10^{-4} m_{e}$, this fixes the $m_{D} / m_{R}$ ratio to be of the $\frac{1}{70}$ for a neutrino mass of $10^{-1} \mathrm{eV}$. And thus the limits from electroweak precision tests are always satisfied. As $y_{N} \propto \operatorname{cosec} \beta$, it is insensitive to $\beta$ for 


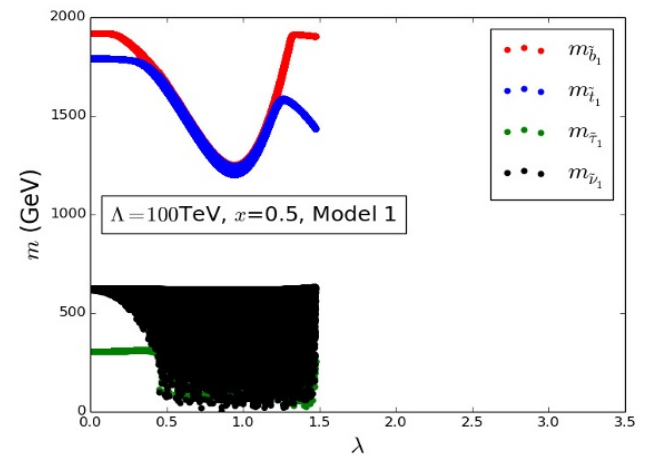

(a)

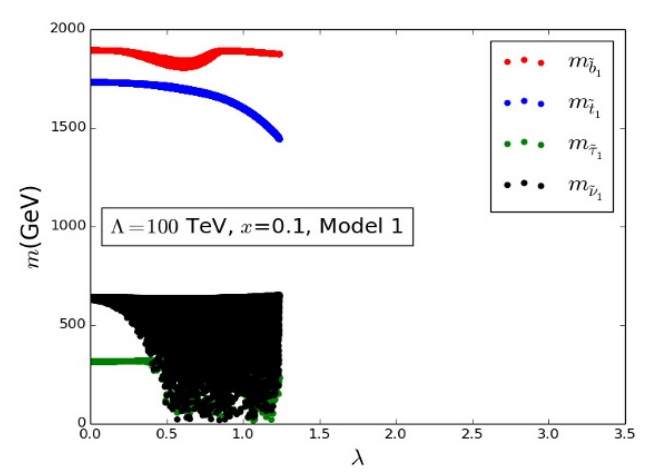

(c)

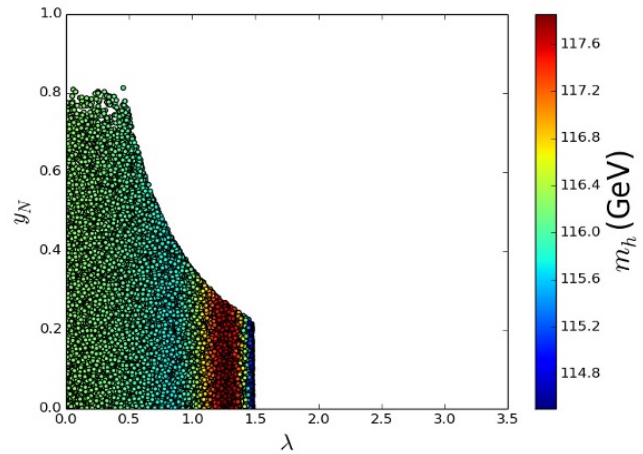

(b)

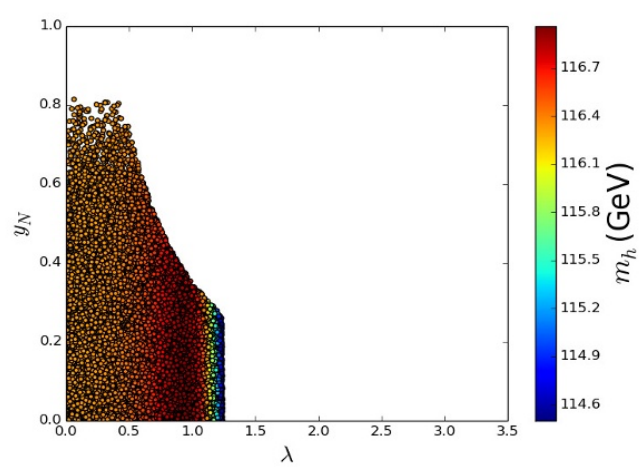

(d)

Figure 1. Model 1: variation of the third generation soft masses with messenger-matter interaction coupling $\lambda$ is shown in the left panel, and Higgs mass values in $\lambda$ and $y_{N}$ plane are shown in the right panel. The upper and lower plots correspond to $x=0.5$ and $x=0.1$ respectively.

higher values of $\tan \beta$. We thus kept $\tan \beta=10$ through out the analysis. For spectrum calculation, a modified version of the publicly available code SuSeFLAV [74] is used. All the low energy phenomenological constraints including flavour constraints dominantly from $B R\left(B \rightarrow X_{s}+\gamma\right)$ and mass constraints from LHC are imposed on the spectrum. We will now discuss each model in detail.

\subsection{Model 1}

Model 1 is a Type II model of our classification which interacts with the quark doublet fields and the singlet. $W_{\text {mix }}=\lambda N^{c} Q \bar{Q}_{m}$, the resultant one-loop and two-loop corrections to the various soft masses are shown below

$$
\begin{aligned}
\delta M_{\tilde{Q}}^{2} & =\left[-\frac{\alpha_{\lambda}}{24 \pi} x^{2} h(x)+\frac{\alpha_{\lambda}\left(-\alpha_{1}+5\left(-9 \alpha_{2}-16 \alpha_{3}+6 \alpha_{N}+24 \alpha_{\lambda}\right)\right)}{240 \pi^{2}}\right] \Lambda^{2} \\
\delta M_{\tilde{U}^{c}}^{2} & =\left[-\frac{\alpha_{t} \alpha_{\lambda}}{8 \pi^{2}}\right] \Lambda^{2} \\
\delta M_{\tilde{D}^{c}}^{2} & =\left[-\frac{\alpha_{b} \alpha_{\lambda}}{8 \pi^{2}}\right] \Lambda^{2}
\end{aligned}
$$




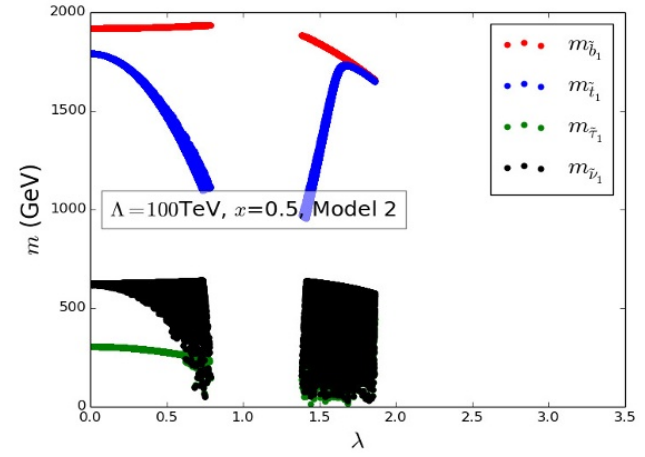

(a)

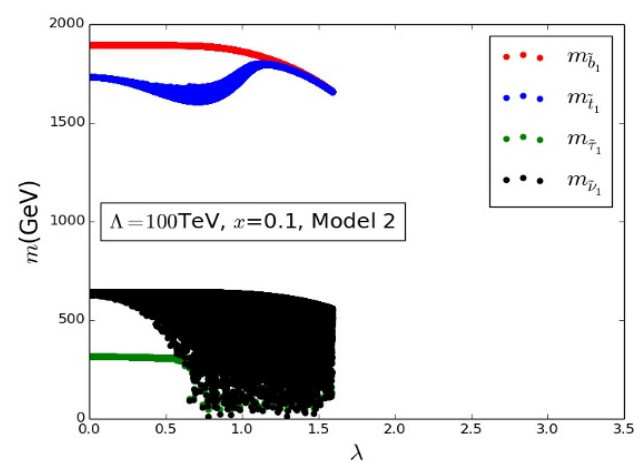

(c)

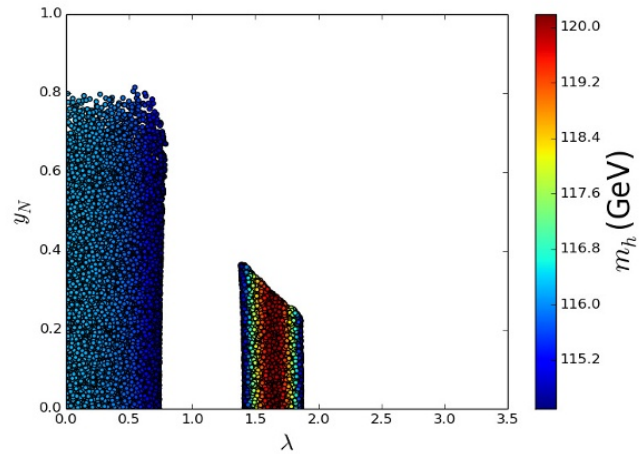

(b)

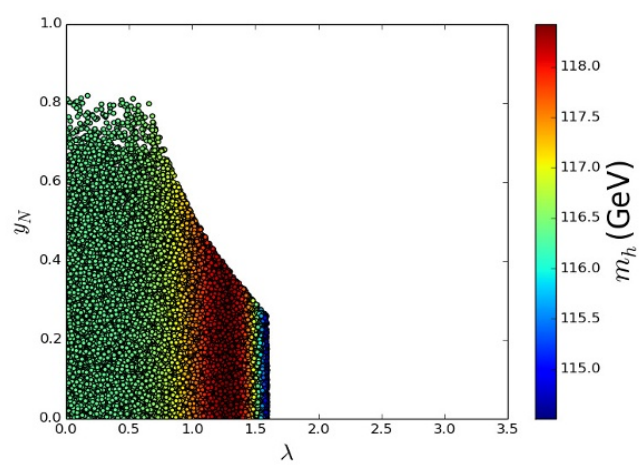

(d)

Figure 2. Model 2: upper two plots correspond to $x=0.5$ and the lower two are for $x=0.1$. Note that depth of blue line is higher for this model compared to model 1. Please see caption of figure 1 for details of notation.

$$
\begin{aligned}
\delta M_{\tilde{L}}^{2} & =\left[-\frac{3 \alpha_{N} \alpha_{\lambda}}{8 \pi^{2}}\right] \Lambda^{2} \\
\delta M_{\tilde{N}^{c}}^{2} & =\left[-\frac{\alpha_{\lambda}}{4 \pi} x^{2} h(x)+\frac{\alpha_{\lambda}\left(-\alpha_{1}+5\left(-9 \alpha_{2}-16 \alpha_{3}+3\left(\alpha_{b}+\alpha_{t}+8 \alpha_{\lambda}\right)\right)\right)}{40 \pi^{2}}\right] \Lambda^{2} \\
\delta M_{H_{u}}^{2} & =\left[-\frac{3\left(\alpha_{t}+2 \alpha_{N}\right) \alpha_{\lambda}}{16 \pi^{2}}\right] \Lambda^{2} \\
\delta M_{H_{d}}^{2} & =\left[-\frac{3 \alpha_{b} \alpha_{\lambda}}{16 \pi^{2}}\right] \Lambda^{2} \\
\delta A_{t} & =\left[-\frac{\alpha_{\lambda}}{4 \pi}\right] \Lambda \\
\delta A_{b} & =\left[-\frac{\alpha_{\lambda}}{4 \pi}\right] \Lambda \\
\delta A_{N} & =\left[-\frac{3 \alpha_{\lambda}}{2 \pi}\right] \Lambda
\end{aligned}
$$


where

$$
\alpha_{t}=\frac{y_{t}^{2}}{4 \pi}, \alpha_{b}=\frac{y_{b}^{2}}{4 \pi}, \alpha_{\tau}=\frac{y_{\tau}^{2}}{4 \pi}, \alpha_{\lambda}=\frac{\lambda^{2}}{4 \pi} \text { and } \alpha_{N}=\frac{y_{N}^{2}}{4 \pi} .
$$

In this model $\tilde{Q}$ and $\tilde{N}^{c}$ get one-loop negative corrections to their masses. For $x=$ 0.5, the interplay between the one-loop, two-loop corrections and the standard GMSB contributions is clearly evident in the lightest stop and sbottom masses shown in figure 1 (a). As can be seen, when $\lambda$ is relatively small, the negative one-loop contributions significantly cancel with the standard GMSB contributions, lowering the lightest eigenvalue to smaller values. The cancellation is maximum around $\lambda \sim 1$. Beyond those values of $\lambda$, positive twoloop contributions start dominating over the one-loop contributions resulting in positive and larger spectra. For $x=0.1$ the one-loop effects are no longer important and the cancellation regions disappear as can be seen from the figure 1(c). For both the cases, as $\lambda$ increases, staus and stops start becoming tachyonic for values $\lambda \gtrsim 1.5$. Remember that the staus receive negative contributions at two-loops from matter-messenger mixing terms at the messenger scale (eq. (4.3)).

The Higgs mass values for the allowed parameter space are presented for $x=0.5$ in figure 1(b) and for $x=0.1$ in figure 1(d). This model can not produce the correct Higgs mass because although both $A_{t}$ and $A_{N}$ are generated but sleptons are not that heavy. However $A_{N}$ is dominant over $A_{t}$ by a factor three as can be seen from eq. (4.3). To disentangle the effects from each contribution, we use three different notations to illustrate the corrections to the Higgs mass. We used $m_{h}^{0}$ parameter for pure MSSM Higgs mass, $m_{h}^{0+\Delta}$ denotes Higgs mass, calculated including the matter messenger mixing terms in the boundary conditions but without considering the corrections from the neutrino-sneutrino sector, on the top of the MSSM and $m_{h}$ is the actual Higgs mass when all the corrections are considered.

Four benchmark points (two each with $x=0.5$ and $x=0.1$ ) are presented in table 2 . As can be seen from the benchmark points, even both $A_{t}$ and $A_{N}$ are not sufficient to provide correct Higgs mass. The stop spectrum is relatively light $\sim 1.7 \mathrm{TeV}$. The sneutrino mixing parameter $A_{N}$ is relatively large compared to the $A_{t}$ generated.

\subsection{Model 2}

This model has similar structure as Model 1 with quark doublets replaced by the up type quark singlet. The resultant one-loop and two-loop corrections are listed below in eq. (4.5)

$$
\begin{aligned}
\delta M_{\tilde{Q}}^{2} & =\left[-\frac{\alpha_{t} \alpha_{\lambda}}{16 \pi^{2}}\right] \Lambda^{2} \\
\delta M_{\tilde{U}^{c}}^{2} & =\left[-\frac{\alpha_{\lambda}}{24 \pi} x^{2} h(x)+\frac{\alpha_{\lambda}\left(-16 \alpha_{1}-80 \alpha_{3}+30 \alpha_{N}+75 \alpha_{\lambda}\right)}{240 \pi^{2}}\right] \Lambda^{2} \\
\delta M_{\tilde{L}}^{2} & =\left[-\frac{3 \alpha_{N} \alpha_{\lambda}}{16 \pi^{2}}\right] \Lambda^{2} \\
\delta M_{\tilde{N}^{c}}^{2} & =\left[-\frac{\alpha_{\lambda}}{8 \pi} x^{2} h(x)+\frac{\alpha_{\lambda}\left(-16 \alpha_{1}-80 \alpha_{3}+30 \alpha_{t}+75 \alpha_{\lambda}\right)}{80 \pi^{2}}\right] \Lambda^{2} \\
\delta M_{H_{u}}^{2} & =\left[-\frac{3\left(\alpha_{t}+\alpha_{N}\right) \alpha_{\lambda}}{16 \pi^{2}}\right] \Lambda^{2}
\end{aligned}
$$




\begin{tabular}{|c|c|c|c|c|c|c|c|c|c|}
\hline Parameter & $\mathrm{x}=0.5$ & $\mathrm{x}=0.5$ & $\mathrm{x}=0.1$ & $\mathrm{x}=0.1$ & Parameter & $\mathrm{x}=0.5$ & $\mathrm{x}=0.5$ & $\mathrm{x}=0.1$ & $\mathrm{x}=0.1$ \\
\hline$\lambda$ & 1.34 & 0.53 & 0.55 & 0.55 & $y_{N}$ & 0.24 & 0.51 & 0.51 & 0.21 \\
\hline$A_{N}$ & -6650.7 & -1021.8 & -1074.6 & -1136.3 & $A_{t}$ & -1477.9 & -727.3 & -829.4 & -843.4 \\
$m_{R}$ & 5823.8 & 6195.3 & 6469.5 & 3732.3 & $\mu$ & 1400. & 790. & 935. & 823. \\
$m_{\tilde{\nu}_{1}}$ & 133. & 441. & 426. & 610. & $m_{\tilde{\nu}_{2,3}} / m_{\nu_{2,3}}$ & 5824. & 6196. & 6470. & 3732. \\
\hline$m_{h}$ & 117.05 & 115.62 & 116.06 & 117.21 & $m_{H}$ & 1523. & 1003. & 1129. & 999. \\
$m_{h}^{0+\Delta}$ & 117.05 & 115.60 & 116.04 & 117.21 & $m_{A^{0}}$ & 1539. & 1013. & 1141. & 1001. \\
$m_{h}^{0}$ & 115.84 & 115.84 & 116.18 & 116.18 & $m_{H^{ \pm}}$ & 1541. & 1016. & 1144. & 1003. \\
\hline$M_{S}$ & 1728. & 1706. & 1768. & 1759. & $m_{\tilde{g}}$ & 2187. & 2180. & 2108. & 2108. \\
$\tilde{t}_{1}$ & 1561. & 1611. & 1707. & 1703. & $\tilde{t}_{2}$ & 1913. & 1806. & 1832. & 1816. \\
$\tilde{b}_{1}$ & 1912. & 1620. & 1831. & 1809. & $\tilde{b}_{2}$ & 1926. & 1928. & 1899. & 1888. \\
$\tilde{\tau}_{1}$ & 119. & 317. & 316. & 311. & $\tilde{\tau}_{2}$ & 299. & 321. & 323. & 324. \\
$\tilde{u}_{1}$ & 1935. & 1916. & 1897. & 1892. & $\tilde{u}_{2}$ & 2003. & 1936. & 1909. & 1910. \\
$\tilde{d}_{1}$ & 1927. & 1931. & 1901. & 1902. & $\tilde{d}_{2}$ & 1993. & 1931. & 1902. & 1902. \\
$\tilde{e}_{1}$ & 299. & 321. & 323. & 324. & $\tilde{e}_{2}$ & 311. & 446. & 432. & 614. \\
$N_{1}$ & 431. & 430. & 412. & 412. & $N_{2}$ & 822. & 749. & 772. & 750. \\
$N_{3}$ & 1408. & 795. & 942. & 829. & $N_{4}$ & 1413. & 878. & 965. & 873. \\
$C_{1}$ & 806. & 740. & 758. & 739. & $C_{2}$ & 1404. & 863. & 955. & 862. \\
\hline
\end{tabular}

Table 2. Model 1: matter-messenger mixing parameter, neutrino sector parameters, Higgs mass and SUSY spectrum for the benchmark points from $x=0.5$ and $x=0.1$ cases. In each case the considered benchmark represents small and large allowed $\lambda$ range. Here $m_{h}^{0}$ means pure MSSM Higgs mass calculated at the two-loop level, $m_{h}^{0+\Delta}$ is the Higgs mass when all the mixing terms except the neutrino mixing are considered on the top of the MSSM and $m_{h}$ is the actual Higgs mass when all the corrections were considered. All the masses are given in $\mathrm{GeV}$. The $\Lambda$ values are taken as in eq. (4.1).

$$
\begin{aligned}
\delta A_{t} & =\left[-\frac{\alpha_{\lambda}}{4 \pi}\right] \Lambda \\
\delta A_{N} & =\left[-\frac{3 \alpha_{\lambda}}{4 \pi}\right] \Lambda .
\end{aligned}
$$

In this case $\tilde{U}^{c}$ and $N^{c}$ fields get one-loop negative and two-loop positive contributions to their soft masses. From figures 2(a) and 2(c), we can see the variation of the lightest third generation mass eigenvalues with respect to $\lambda$. For $x=0.5$, cancellations only appear for the stop sector and not in the bottom sector as the messenger matter interactions are only active for the up-type singlet sector. The cancellations are however much deeper here as the standard GMSB contributions for the singlet up squarks is lesser compared to the doublet squarks. The cancellation is milder as expected for $x=0.1$.

In this case $A_{N}$ dominates $A_{t}$ by a factor three as in Model 1 . For large $\lambda \gtrsim 2$, sneutrinos, stops and sbottoms become tachyonic making the model unviable. The Higgs mass values are presented in figures $2(\mathrm{~b})$ and $2(\mathrm{~d})$. 


\begin{tabular}{|c|c|c|c|c|c|c|c|c|c|}
\hline Parameter & $\mathrm{x}=0.5$ & $\mathrm{x}=0.5$ & $\mathrm{x}=0.1$ & $\mathrm{x}=0.1$ & Parameter & $\mathrm{x}=0.5$ & $\mathrm{x}=0.5$ & $\mathrm{x}=0.1$ & $\mathrm{x}=0.1$ \\
\hline$\lambda$ & 1.58 & 0.66 & 1.32 & 0.72 & $y_{N}$ & 0.27 & 0.62 & 0.36 & 0.65 \\
\hline$A_{N}$ & -4599.7 & -785.0 & -3137.3 & -882.2 & $A_{t}$ & -1845.6 & -814.3 & -1495.5 & -925.6 \\
$m_{R}$ & 6162.5 & 8773.4 & 7394.6 & 9977.0 & $\mu$ & 1551. & 852. & 1527. & 1050. \\
$m_{\tilde{\nu}_{1}}$ & 306. & 425. & 115. & 322. & $m_{\tilde{\nu}_{2,3}} / m_{\nu_{2,3}}$ & 6163. & 8774. & 7395. & 9978. \\
\hline$m_{h}$ & 119.24 & 114.84 & 117.80 & 116.24 & $m_{H}$ & 1671. & 1053. & 1643. & 1217. \\
$m_{h}^{0+\Delta}$ & 119.22 & 114.82 & 117.78 & 116.22 & $m_{A^{0}}$ & 1686. & 1071. & 1662. & 1241. \\
$m_{h}^{0}$ & 119.22 & 114.82 & 115.95 & 115.94 & $m_{H^{ \pm}}$ & 1688. & 1074. & 1664. & 1244. \\
\hline$M_{S}$ & 1722. & 1569. & 1820. & 1761. & $m_{\tilde{g}}$ & 2182. & 2184. & 2114. & 2110. \\
$\tilde{t}_{1}$ & 1600. & 1291. & 1771. & 1666. & $\tilde{t}_{2}$ & 1853. & 1906. & 1871. & 1862. \\
$\tilde{b}_{1}$ & 1808. & 1928. & 1783. & 1888. & $\tilde{b}_{2}$ & 1922. & 1934. & 1889. & 1901. \\
$\tilde{\tau}_{1}$ & 269. & 256. & 108. & 288. & $\tilde{\tau}_{2}$ & 321. & 262. & 418. & 315. \\
$\tilde{u}_{1}$ & 1922. & 1948. & 1885. & 1910. & $\tilde{u}_{2}$ & 1935. & 1974. & 1990. & 1927. \\
$\tilde{d}_{1}$ & 1930. & 1934. & 1896. & 1901. & $\tilde{d}_{2}$ & 1930. & 1959. & 1897. & 1909. \\
$\tilde{e}_{1}$ & 321. & 262. & 418. & 315. & $\tilde{e}_{2}$ & 356. & 430. & 422. & 349. \\
$N_{1}$ & 431. & 430. & 413. & 412. & $N_{2}$ & 822. & 783. & 782. & 780. \\
$N_{3}$ & 1559. & 858. & 1536. & 1058. & $N_{4}$ & 1563. & 908. & 1540. & 1071. \\
$C_{1}$ & 806. & 769. & 773. & 766. & $C_{2}$ & 1554. & 895. & 1529. & 1062. \\
\hline
\end{tabular}

Table 3. Benchmark points for Model 2. As can be seen, the neutrino corrections can be significant in some regions of the parameter space compared to the matter messenger mixing corrections. See caption of table 2 for details of notation.

Four benchmark points are given in table 3, as before two for $x=0.5$ and two for $x=0.1$. As can be seen from the points, neutrino/sneutrino contribution is not significant in this model.

\subsection{Model 3}

Model 3 is a Type II model of our classification in which down quark interacts with a messenger field transforming as a conjugate representation of $D^{c}: W_{\text {mix }}=\lambda N^{c} D^{c} \bar{D}_{m}^{c}$. The resultant one-loop and two-loop corrections to the various soft masses are shown below

$$
\begin{aligned}
\delta M_{\tilde{Q}}^{2} & =\left[-\frac{3 \alpha_{b} \alpha_{\lambda}}{16 \pi^{2}}\right] \Lambda^{2} \\
\delta M_{\tilde{D}^{c}}^{2} & =\left[-\frac{\alpha_{\lambda}}{8 \pi} x^{2} h(x)+\frac{\alpha_{\lambda}\left(-4 \alpha_{1}-80 \alpha_{3}+30 \alpha_{N}+195 \alpha_{\lambda}\right)}{80 \pi^{2}}\right] \Lambda^{2} \\
\delta M_{\tilde{L}}^{2} & =\left[-\frac{9 \alpha_{N} \alpha_{\lambda}}{16 \pi^{2}}\right] \Lambda^{2} \\
\delta M_{H_{u}}^{2} & =\left[-\frac{9 \alpha_{N} \alpha_{\lambda}}{16 \pi^{2}}\right] \Lambda^{2}
\end{aligned}
$$




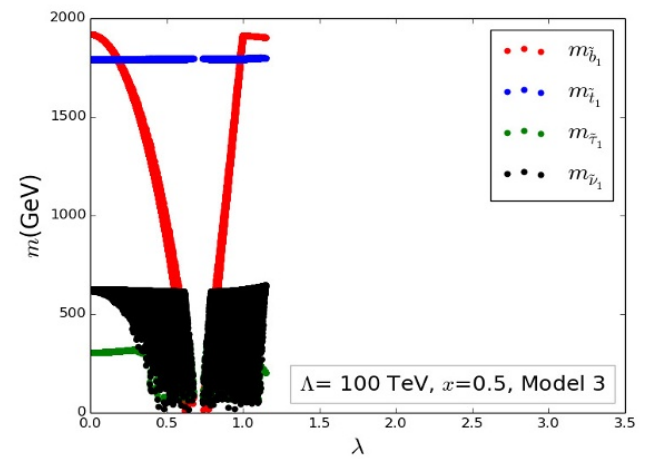

(a)

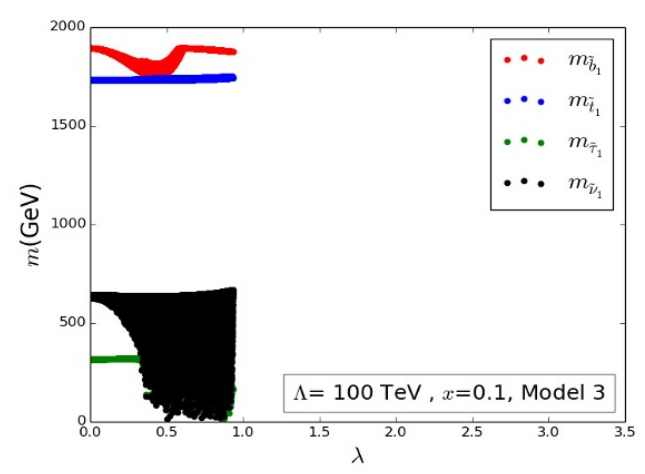

(c)

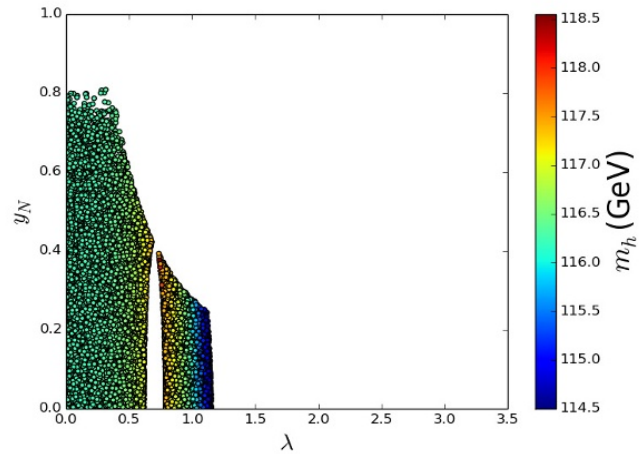

(b)

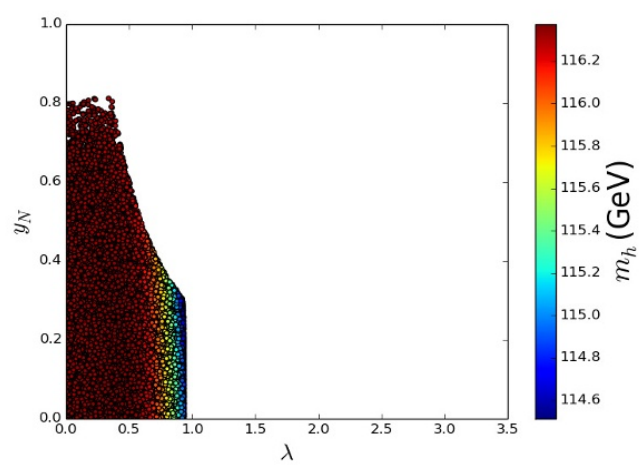

(d)

Figure 3. Model 3: spectrum variation with $\lambda$ and Higgs mass data points in $y_{N}$ and $\lambda$ plane. Note that in (b) origin is not at zero but at 0.7 for $\lambda$. Please see caption of figure 1 for details of notations.

$$
\begin{aligned}
\delta M_{H_{d}}^{2} & =\left[-\frac{9 \alpha_{b} \alpha_{\lambda}}{16 \pi^{2}}\right] \Lambda^{2} \\
\delta M_{\tilde{N}^{c}}^{2} & =\left[-3 \frac{\alpha_{\lambda}}{8 \pi} x^{2} h(x)+\frac{3 \alpha_{\lambda}\left(-4 \alpha_{1}-80 \alpha_{3}+30 \alpha_{b}+195 \alpha_{\lambda}\right)}{80 \pi^{2}}\right] \Lambda^{2} \\
\delta A_{b} & =\left[-\frac{3 \alpha_{\lambda}}{4 \pi}\right] \Lambda \\
\delta A_{N} & =\left[-\frac{9 \alpha_{\lambda}}{4 \pi}\right] \Lambda .
\end{aligned}
$$

Here $M_{\tilde{D}^{c}}$ and $M_{\tilde{N}^{c}}$ get one-loop negative contributions. Cancellation between the oneloop contribution and the two-loop contributions for $M_{\tilde{D}^{c}}$ is so severe in $x=0.5$ case that the $m_{\tilde{b}_{1}}$ becomes tachyonic for $\lambda \approx 0.6-0.7$ (figure $3(\mathrm{a})$ ). One may wonder why $m_{\tilde{b}_{1}}$ goes to negative here while $m_{\tilde{t}_{1}}$ of previous model does not touch the zero line. Note that $\tilde{U}^{c}$ mass at the boundary is slightly higher than that of $\tilde{D}^{c}$ because former has hypercharge double of the later one. Hence the cancellation is less severe in model 2. This cancellation is less for $x=0.1$ as expected (figure 3(c)).

In this model, no $A_{t}$ term is generated at the boundary. We see that parameter space of $\lambda$ splits into two parts for $x=0.5$ (figure $3(\mathrm{~b})$ ) and one can not obtain the correct Higgs 


\begin{tabular}{|c|c|c|c|c|c|c|c|c|c|}
\hline Parameter & $\mathrm{x}=0.5$ & $\mathrm{x}=0.5$ & $\mathrm{x}=0.1$ & $\mathrm{x}=0.1$ & Parameter & $\mathrm{x}=0.5$ & $\mathrm{x}=0.5$ & $\mathrm{x}=0.1$ & $\mathrm{x}=0.1$ \\
\hline$\lambda$ & 0.83 & 0.41 & 0.81 & 0.45 & $y_{N}$ & 0.28 & 0.54 & 0.32 & 0.59 \\
\hline$A_{N}$ & -3921.8 & -955.1 & -3716.3 & -1072.6 & $A_{t}$ & -572.2 & -575.5 & -679.5 & -679.6 \\
$m_{R}$ & 4753.9 & 8559.6 & 9412.0 & 7235.1 & $\mu$ & 803. & 770. & 937. & 905. \\
$m_{\tilde{\nu}_{1}}$ & 330. & 421. & 273. & 325. & $m_{\tilde{\nu}_{2,3}} / m_{\nu_{2,3}}$ & 4754. & 8560. & 9412. & 7236. \\
\hline$m_{h}$ & 117.22 & 116.43 & 115.48 & 116.30 & $m_{H}$ & 1004. & 983. & 1111. & 1098. \\
$m_{h}^{0+\Delta}$ & 117.20 & 116.42 & 115.46 & 116.27 & $m_{A^{0}}$ & 1011. & 997. & 1135. & 1115. \\
$m_{h}^{0}$ & 116.23 & 116.22 & 116.33 & 116.33 & $m_{H^{ \pm}}$ & 1014. & 1000. & 1137. & 1117. \\
\hline$M_{S}$ & 1842. & 1842. & 1803. & 1798. & $m_{\tilde{g}}$ & 2183. & 2182. & 2117. & 2110. \\
$\tilde{t}_{1}$ & 1792. & 1792. & 1744. & 1738. & $\tilde{t}_{2}$ & 1893. & 1893. & 1864. & 1861. \\
$\tilde{b}_{1}$ & 694. & 1266. & 1885. & 1800. & $\tilde{b}_{2}$ & 1921. & 1923. & 1894. & 1897. \\
$\tilde{\tau}_{1}$ & 317. & 326. & 233. & 299. & $\tilde{\tau}_{2}$ & 344. & 332. & 259. & 324. \\
$\tilde{u}_{1}$ & 1927. & 1927. & 1912. & 1906. & $\tilde{u}_{2}$ & 1971. & 1971. & 1938. & 1942. \\
$\tilde{d}_{1}$ & 1926. & 1924. & 1927. & 1899. & $\tilde{d}_{2}$ & 1960. & 1956. & 1994. & 1925. \\
$\tilde{e}_{1}$ & 344. & 332. & 259. & 324. & $\tilde{e}_{2}$ & 361. & 427. & 299. & 350. \\
$N_{1}$ & 430. & 429. & 413. & 412. & $N_{2}$ & 756. & 736. & 772. & 768. \\
$N_{3}$ & 808. & 775. & 943. & 912. & $N_{4}$ & 881. & 870. & 966. & 938. \\
$C_{1}$ & 747. & 727. & 759. & 755. & $C_{2}$ & 868. & 855. & 956. & 928. \\
\hline
\end{tabular}

Table 4. Benchmark points for Model 3. See caption of table 2 for details of notation.

mass in this model. For the case of $x=0.1$, however, parameter space for $\lambda$ is continuous (figure 3(d)) and the spectrum becomes tachyonic for $\lambda>1.0$. The benchmark points are given in table 4.

\subsection{Model 4}

This is a Type II model with messenger matter interaction $W_{\text {mix }}=\lambda N^{c} L H_{u}^{m}$. Like the previous model, this model has $5 \oplus \overline{5}$ messengers. One-loop and two-loop contribution to the soft masses are shown in eq. (4.7).

$$
\begin{aligned}
\delta M_{\tilde{L}}^{2} & =\left[-\frac{\alpha_{\lambda}}{8 \pi} x^{2} h(x)+\frac{3 \alpha_{\lambda}\left(-3 \alpha_{1}-15 \alpha_{2}+10\left(\alpha_{N}+5 \alpha_{\lambda}\right)\right)}{80 \pi^{2}}\right] \Lambda^{2} \\
\delta M_{\tilde{E}^{c}}^{2} & =\left[-\frac{3 \alpha_{\lambda} \alpha_{\tau}}{8 \pi^{2}}\right] \Lambda^{2} \\
\delta M_{H_{u}}^{2} & =\left[-\frac{9 \alpha_{N} \alpha_{\lambda}}{16 \pi^{2}}\right] \Lambda^{2} \\
\delta M_{H_{d}}^{2} & =\left[-\frac{3 \alpha_{\lambda} \alpha_{\tau}}{16 \pi^{2}}\right] \Lambda^{2} \\
\delta M_{\tilde{N}^{c}}^{2} & =\left[-\frac{\alpha_{\lambda}}{4 \pi} x^{2} h(x)+\frac{3 \alpha_{\lambda}\left(5\left(-3 \alpha_{2}+2 \alpha_{N}+10 \alpha_{\lambda}+\alpha_{\tau}\right)-3 \alpha_{1}\right)}{40 \pi^{2}}\right] \Lambda^{2}
\end{aligned}
$$




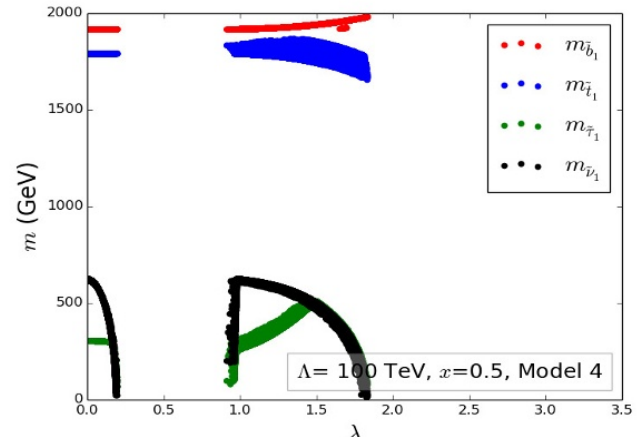

(a)

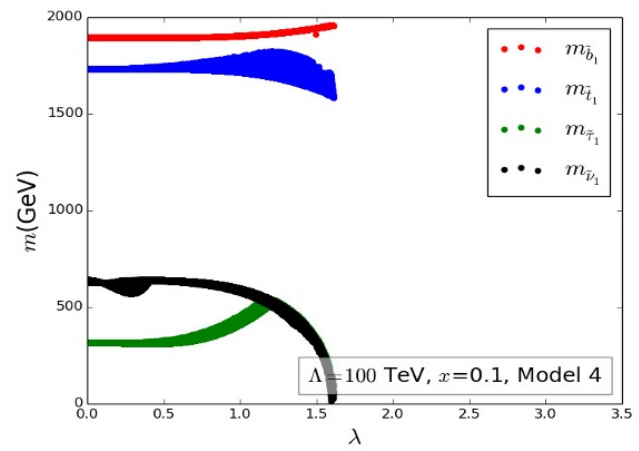

(c)

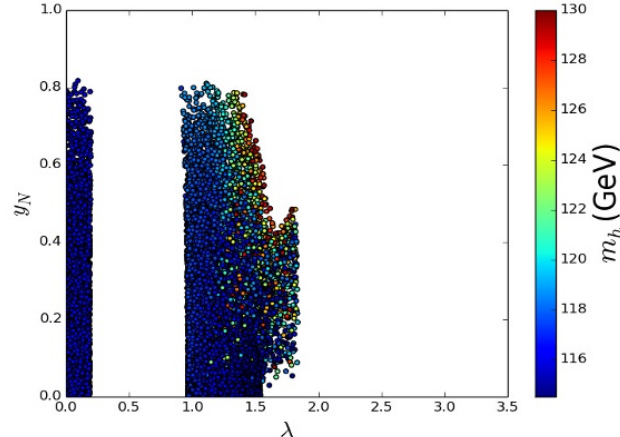

(b)

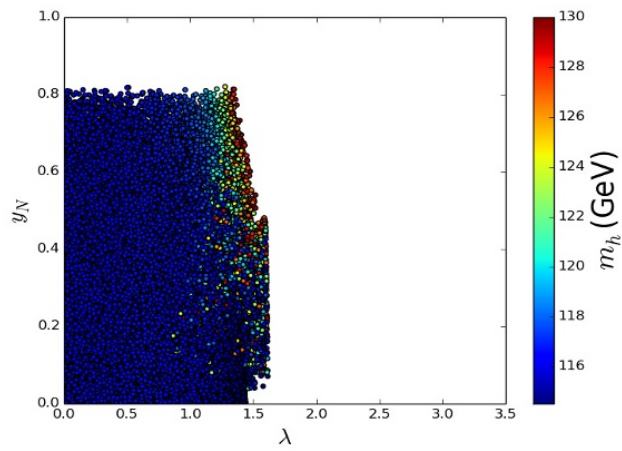

(d)

Figure 4. Model 4: spectrum variation with $\lambda$ and Higgs mass values in $y_{N}$ and $\lambda$ plane. Please see caption of figure 1 for details of notation.

$$
\begin{aligned}
\delta A_{\tau} & =\left[-\frac{3 \alpha_{\lambda}}{4 \pi}\right] \Lambda \\
\delta A_{N} & =\left[-\frac{9 \alpha_{\lambda}}{4 \pi}\right] \Lambda .
\end{aligned}
$$

Here $M_{\tilde{L}}^{2}$ and $M_{\tilde{N}^{c}}^{2}$ get one-loop negative contributions and two-loop positive contributions. We know that usual GMSB contributions to the $M_{\tilde{L}^{2}}^{2}$ and $M_{\tilde{E}^{c}}^{2}$ are small. Thus in the models where these masses get one-loop negative contributions, $M_{\tilde{L}}^{2}$ will be large (comparable to $m_{R}$ ). Therefore $d_{1,2}$ (eq. (3.11) and (3.12)) will be small which appear in the denominator of the Higgs mass expressions and enhances the contribution. Therefore one will correct Higgs mass.

In figure 4 (a) one can see that $m_{\tilde{\tau}_{1}}$ becomes tachyonic for vary small values of $\lambda \sim 0.18$ and for larger $\lambda$ one can get positive spectrum. The larger band $(\lambda>1.0)$ has correct Higgs mass. For $x=0.1$, strength of one-loop contribution diminishes and hence larger values of $\lambda$ are allowed. Beyond $\lambda=1.6, m_{\tilde{\tau}_{1}}$ is tachyonic as can be seen in figure 4(c). For these values of $\lambda$ two-loop negative contribution to $M_{\tilde{E}^{c}}$ dominates over usual GMSB contribution. In the Higgs mass scatter plot (figure 4(b)) and (d)), one can see that this model is capable of 


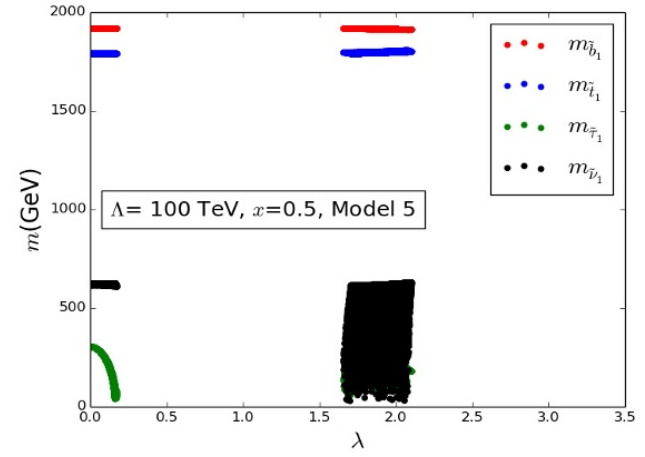

(a)

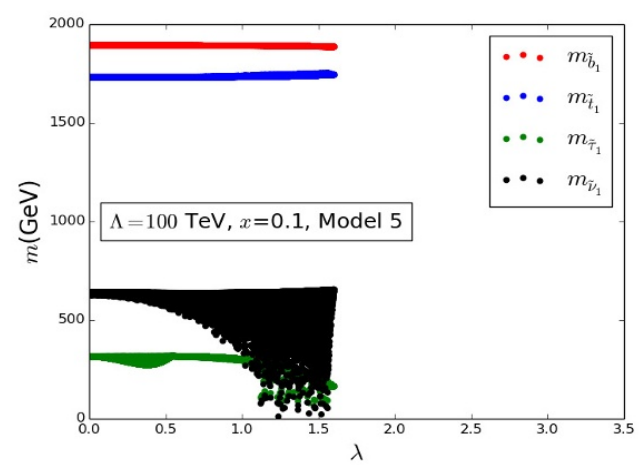

(c)

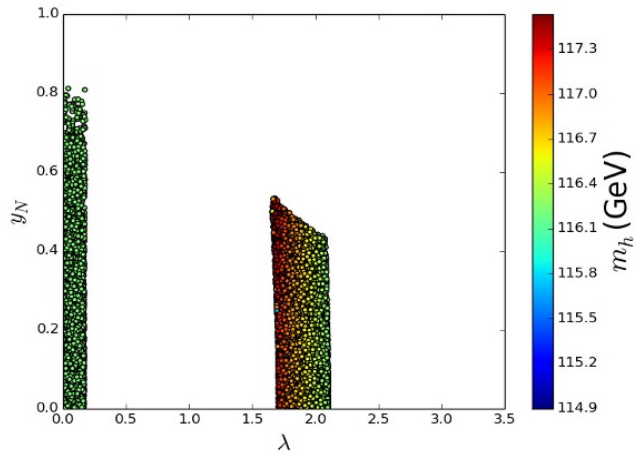

(b)

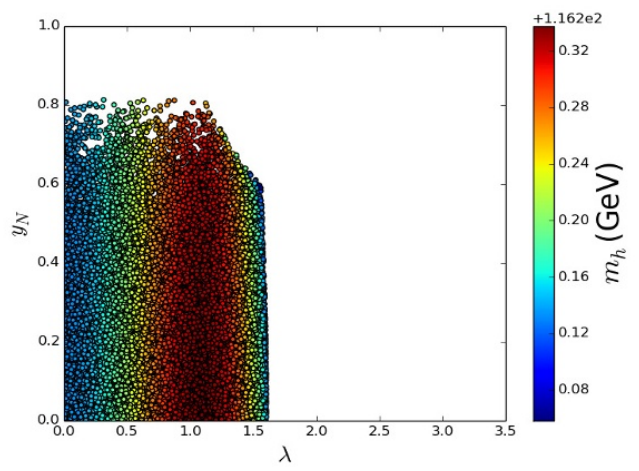

(d)

Figure 5. Model 5: spectrum variation with $\lambda$ and Higgs mass values in $y_{N}$ and $\lambda$ plane. Please see caption of figure 1 for details of notation.

giving correct Higgs mass. From these figures, we can see the importance of $m_{R}$ parameter. The Higgs mass is sensitive to the $m_{R}$ value for a given point in the $y_{N^{-}} \lambda$ plane. Like the model 3 , in this model too $A_{t}$ term is not generated at the boundary. Hence, $A_{N}$ and heavy $M_{\tilde{L}}^{2}$ are mainly responsible for raising the Higgs mass up to the required value. For the $x=0.5$ case, one need the value of $-A_{N}$ is $\sim 12$ to $4 \mathrm{TeV}$ (table 5 ) to raise the Higgs mass from its pure MSSM value as shown in table 5 .

\subsection{Model 5}

This model represents the interaction of $N^{c}$ and $E^{c}$ field with the messenger field $E_{m}^{c}$ so consequently $N^{c}$ and $E^{c}$ get negative corrections at one-loop and positive two-loop corrections. The other fields interacting with them receive two-loop corrections, as given below

$$
\begin{aligned}
\delta M_{\tilde{L}}^{2} & =\left[-\frac{\alpha_{\lambda}\left(\alpha_{N}+\alpha_{\tau}\right)}{16 \pi^{2}}\right] \Lambda^{2} \\
\delta M_{\tilde{E}^{c}}^{2} & =\left[-\frac{\alpha_{\lambda}}{24 \pi} x^{2} h(x)+\frac{\alpha_{\lambda}\left(-12 \alpha_{1}+10 \alpha_{N}+15 \alpha_{\lambda}\right)}{80 \pi^{2}}\right] \Lambda^{2}
\end{aligned}
$$




\begin{tabular}{|c|c|c|c|c|c|c|c|c|c|}
\hline Parameter & $\mathrm{x}=0.5$ & $\mathrm{x}=0.5$ & $\mathrm{x}=0.1$ & $\mathrm{x}=0.1$ & Parameter & $\mathrm{x}=0.5$ & $\mathrm{x}=0.5$ & $\mathrm{x}=0.1$ & $\mathrm{x}=0.1$ \\
\hline$\lambda$ & 1.49 & 1.18 & 1.37 & 0.82 & $y_{N}$ & 0.39 & 0.14 & 0.46 & 0.17 \\
\hline$A_{N}$ & -12389.9 & -7925.4 & -10240.4 & -3869.9 & $A_{t}$ & -513. & -578.0 & -581.2 & -693.0 \\
$m_{R}$ & 7564.6 & 2891.9 & 7985.1 & 2096.7 & $\mu$ & 1624. & 743. & 1893. & 798. \\
$m_{\tilde{\nu}_{1}}$ & 5801. & 2732. & 6190. & 2161. & $m_{\tilde{\nu}_{2,3}} / m_{\nu_{2,3}}$ & 7567. & 2900. & 7987. & 2079. \\
\hline$m_{h}$ & 124.93 & 122.69 & 125.04 & 124.53 & $m_{H}$ & 1558. & 949. & 1767. & 1011. \\
$m_{h}^{0+\Delta}$ & 112.79 & 116.08 & 112.59 & 116.15 & $m_{A^{0}}$ & 1709. & 953. & 1953. & 1013. \\
$m_{h}^{0}$ & 116.20 & 116.2 & 116.33 & 116.3 & $m_{H^{ \pm}}$ & 1709. & 955. & 1953. & 1016. \\
\hline$M_{S}$ & 1821. & 1834. & 1767. & 1789. & $m_{\tilde{g}}$ & 2189. & 2188. & 2113. & 2111. \\
$\tilde{t}_{1}$ & 1783. & 1783. & 1727. & 1726. & $\tilde{t}_{2}$ & 1860. & 1887. & 1807. & 1854. \\
$\tilde{b}_{1}$ & 1939. & 1922. & 1928. & 1897. & $\tilde{b}_{2}$ & 1945. & 1927. & 1936. & 1903. \\
$\tilde{\tau}_{1}$ & 501. & 324. & 436. & 350. & $\tilde{\tau}_{2}$ & 5804. & 2741. & 6194. & 2139. \\
$\tilde{u}_{1}$ & 1879. & 1919. & 1827. & 1899. & $\tilde{u}_{2}$ & 1992. & 1971. & 1981. & 1942. \\
$\tilde{d}_{1}$ & 1945. & 1927. & 1936. & 1903. & $\tilde{d}_{2}$ & 1985. & 1957. & 1975. & 1926. \\
$\tilde{e}_{1}$ & 520. & 389. & 652. & 383. & $\tilde{e}_{2}$ & 612. & 605. & 735. & 626. \\
$N_{1}$ & 431. & 429. & 413. & 411. & $N_{2}$ & 824. & 716. & 791. & 738. \\
$N_{3}$ & 1633. & 748. & 1893. & 803. & $N_{4}$ & 1637. & 864. & 1895. & 861. \\
$C_{1}$ & 805. & 707. & 773. & 726. & $C_{2}$ & 1626. & 847. & 1893. & 848. \\
\hline
\end{tabular}

Table 5. Benchmark points for Model 4. See caption of table 2 for details of notation.

$$
\begin{aligned}
\delta M_{\tilde{N}^{c}}^{2} & =\left[-\frac{\alpha_{\lambda}}{24 \pi} x^{2} h(x)+\frac{\alpha_{\lambda}\left(-12 \alpha_{1}+15 \alpha_{\lambda}+10 \alpha_{\tau}\right)}{80 \pi^{2}}\right] \Lambda^{2} \\
\delta M_{H_{u}}^{2} & =\left[-\frac{\alpha_{N} \alpha_{\lambda}}{16 \pi^{2}}\right] \Lambda^{2} \\
\delta M_{H_{d}}^{2} & =\left[-\frac{\alpha_{\lambda} \alpha_{\tau}}{16 \pi^{2}}\right] \Lambda^{2} \\
\delta A_{\tau} & =\left[-\frac{\alpha_{\lambda}}{4 \pi}\right] \Lambda \\
\delta A_{N} & =\left[-\frac{\alpha_{\lambda}}{4 \pi}\right] \Lambda .
\end{aligned}
$$

As can be seen from the above, eq. (4.8), this model leaves the stop sector including $A_{t}$ untouched. The variation of the lightest sparticles eigenvalues with $\lambda$ are presented in figure 5(a). As can be seen from the figure, for $x=0.5$, the cancellations are really strong and there are two distinct regions of $\lambda$ which are viable phenomenologically: $\lambda \lesssim 0.2$ and $1.7 \lesssim \lambda \lesssim 2.25$. For lower values of the $\lambda$, the stau becomes tachyonic as the oneloop contributions from matter messenger mixing lead to significant cancellations in the boundary conditions. For larger values of $\lambda \gtrsim 2.0$, the $A_{\tau}$ becomes large leading again to tachyonic spectrum of the $\tilde{\tau}$. The region with $\lambda \lesssim 0.2$ is irrelevant phenomenologically and thus not pursued further. From figure 5 (c) we see that the cancellations are extremely 


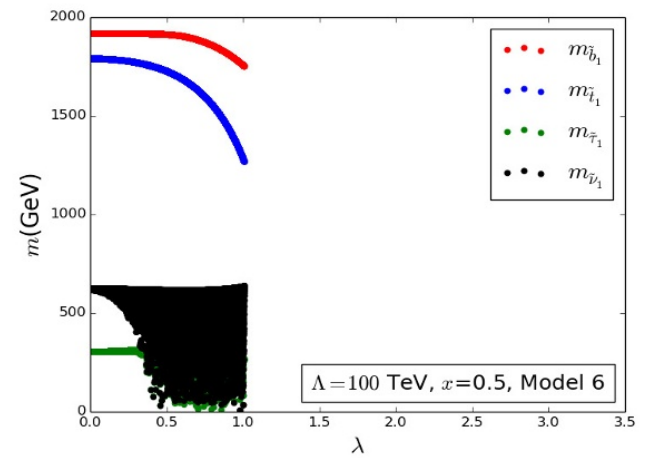

(a)

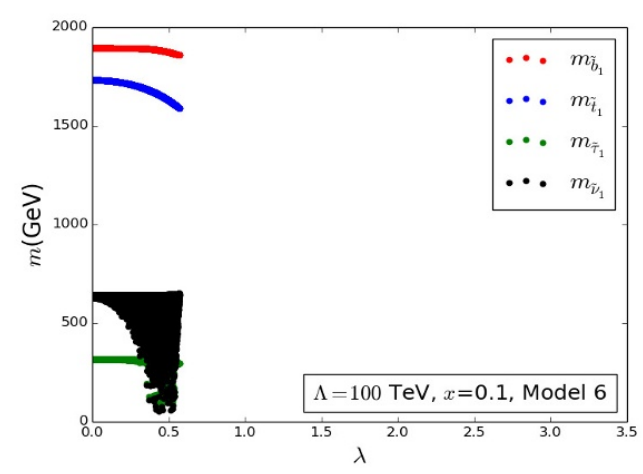

(c)

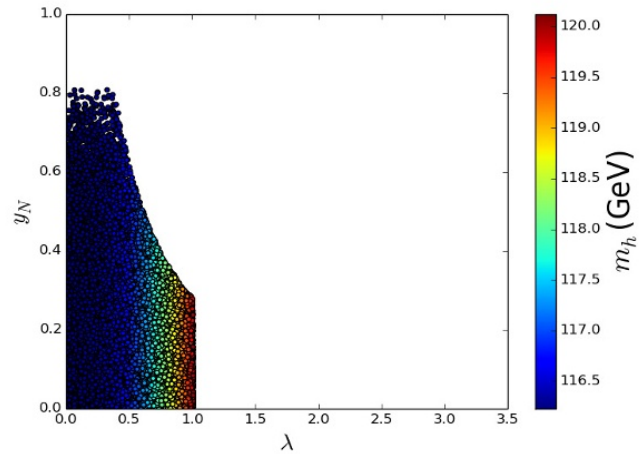

(b)

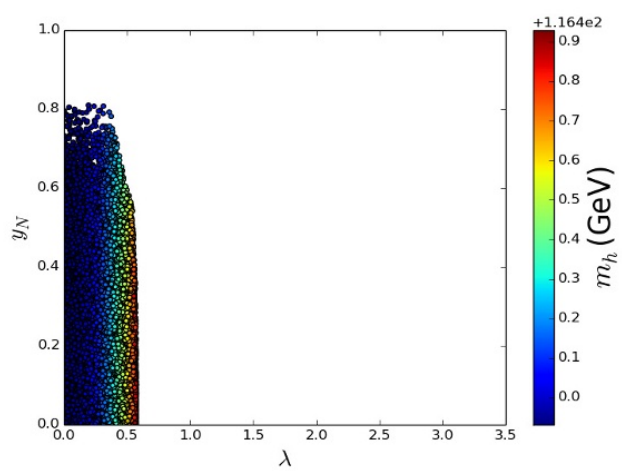

(d)

Figure 6. Model 6: spectrum variation with $\lambda$ and Higgs mass values in $y_{N}$ and $\lambda$ plane. Please see caption of figure 1 for details of notation.

mild and almost disappear for $x=0.1$. For $\lambda \gtrsim 1.5$, the spectrum turns tachyonic and thus unphysical.

The Higgs mass values are shown in figures 5(b) and $5(\mathrm{~d})$ for $x=0.5$ and $x=0.1$ respectively. Unlike in Models 1 and 2, $A_{N}$ does not have a factor three in its messengermatter contributions, eq. (4.8). Since sleptons are not heavy so this model is also not able to produce correct Higgs mass.

In table 6 , the spectra for four benchmark points are shown. It can be seen that for all the points, Higgs mass is $116 \mathrm{GeV}$ as $A_{N}$ is not sufficiently large and sleptons are light.

\subsection{Model 6}

This model represents the interaction $W_{\text {mix }}=\lambda N^{c} H_{u} H_{d}^{m}$. This is also a Type II model. One-loop and two-loop corrections at the boundary are given below:

$$
\begin{aligned}
\delta M_{\tilde{Q}}^{2} & =\left[-\frac{3 \alpha_{t} \alpha_{\lambda}}{16 \pi^{2}}\right] \Lambda^{2} \\
\delta M_{\tilde{U} c}^{2} & =\left[-\frac{3 \alpha_{t} \alpha_{\lambda}}{8 \pi^{2}}\right] \Lambda^{2}
\end{aligned}
$$




\begin{tabular}{|c|c|c|c|c|c|c|c|c|c|}
\hline Parameter & $\mathrm{x}=0.5$ & $\mathrm{x}=0.5$ & $\mathrm{x}=0.1$ & $\mathrm{x}=0.1$ & Parameter & $\mathrm{x}=0.5$ & $\mathrm{x}=0.5$ & $\mathrm{x}=0.1$ & $\mathrm{x}=0.1$ \\
\hline$\lambda$ & 2.00 & 1.79 & 1.46 & 1.02 & $y_{N}$ & 0.40 & 0.45 & 0.47 & 0.28 \\
\hline$A_{N}$ & -2491.1 & -1974.5 & -1303.8 & -683.0 & $A_{t}$ & -569.4 & -569.3 & -685.0 & -698.0 \\
$m_{R}$ & 5960.8 & 5980.6 & 6631.0 & 4818.2 & $\mu$ & 848. & 836. & 884. & 765. \\
$m_{\tilde{\nu}_{1}}$ & 271. & 260. & 435. & 611. & $m_{\tilde{\nu}_{2,3}} / m_{\nu_{2,3}}$ & 5961. & 5981. & 6632. & 4818. \\
\hline$m_{h}$ & 116.11 & 116.64 & 116.04 & 116.15 & $m_{H}$ & 1052. & 1038. & 1096. & 1004. \\
$m_{h}^{0+\Delta}$ & 116.08 & 116.60 & 116.03 & 116.14 & $m_{A^{0}}$ & 1066. & 1052. & 1108. & 1005. \\
$m_{h}^{0}$ & 115.84 & 115.84 & 115.94 & 115.95 & $m_{H^{ \pm}}$ & 1068. & 1055. & 1110. & 1008. \\
\hline$M_{S}$ & 1851. & 1847. & 1806. & 1797. & $m_{\tilde{g}}$ & 2188. & 2188. & 2112. & 2111. \\
$\tilde{t}_{1}$ & 1804. & 1798. & 1747. & 1735. & $\tilde{t}_{2}$ & 1901. & 1897. & 1867. & 1861. \\
$\tilde{b}_{1}$ & 1913. & 1916. & 1888. & 1892. & $\tilde{b}_{2}$ & 1917. & 1920. & 1893. & 1898. \\
$\tilde{\tau}_{1}$ & 221. & 262. & 216. & 300. & $\tilde{\tau}_{2}$ & 221. & 285. & 216. & 300. \\
$\tilde{u}_{1}$ & 1936. & 1931. & 1916. & 1909. & $\tilde{u}_{2}$ & 1969. & 1970. & 1940. & 1940. \\
$\tilde{d}_{1}$ & 1917. & 1920. & 1894. & 1898. & $\tilde{d}_{2}$ & 1955. & 1956. & 1923. & 1923. \\
$\tilde{e}_{1}$ & 275. & 285. & 438. & 612. & $\tilde{e}_{2}$ & 647. & 634. & 665. & 648. \\
$N_{1}$ & 431. & 430. & 412. & 411. & $N_{2}$ & 779. & 774. & 764. & 720. \\
$N_{3}$ & 854. & 842. & 890. & 770. & $N_{4}$ & 904. & 898. & 920. & 845. \\
$C_{1}$ & 768. & 763. & 751. & 711. & $C_{2}$ & 892. & 886. & 910. & 832. \\
\hline
\end{tabular}

Table 6. Benchmark points for Model 5. See caption of table 2 for details of notation.

$$
\begin{aligned}
\delta M_{\tilde{L}}^{2} & =\left[-\frac{9 \alpha_{N} \alpha_{\lambda}}{16 \pi^{2}}\right] \Lambda^{2} \\
\delta M_{H_{u}}^{2} & =\left[-\frac{\alpha_{\lambda}}{8 \pi} x^{2} h(x)+\frac{3 \alpha_{\lambda}\left(-3 \alpha_{1}-15 \alpha_{2}+10\left(\alpha_{N}+5 \alpha_{\lambda}\right)\right)}{80 \pi^{2}}\right] \Lambda^{2} \\
\delta M_{\tilde{N}^{c}}^{2} & =\left[-\frac{\alpha_{\lambda}}{4 \pi} x^{2} h(x)+\frac{3 \alpha_{\lambda}\left(5\left(-3 \alpha_{2}+2 \alpha_{N}+3 \alpha_{t}+10 \alpha_{\lambda}\right)-3 \alpha_{1}\right)}{40 \pi^{2}}\right] \Lambda^{2} \\
\delta A_{t} & =\left[-\frac{3 \alpha_{\lambda}}{4 \pi}\right] \Lambda \\
\delta A_{N} & =\left[-\frac{9 \alpha_{\lambda}}{4 \pi}\right] \Lambda .
\end{aligned}
$$

In this model, two-loop messenger-matter contributions to $\tilde{Q}, \tilde{U}^{c}$ and $\tilde{L}$ masses are negative whereas $\delta M_{H_{u}}^{2}$ gets both negative and positive contribution. For $x=0.5$ case the maximum allowed value for $\lambda$ is $\sim 1.0$ as shown in figure 6(a). In figure 6(b), Higgs mass values are shown in the $\lambda$ vs $y_{N}$ plane. One can not achieve correct Higgs mass in this model.

For $x=0.1$, one-loop effect is small and thus $M_{H_{u}}^{2}$ becomes more massive for increasing $\lambda$. But we cannot consider very large $\lambda$ value because above certain value electro-weak symmetry breaking (EWSB) will be impossible. The allowed value of $\lambda$ for successful EWSB is $\lesssim 0.6$ as shown in figure $6(\mathrm{c})$. As allowed values of $\lambda$ are small, $A_{N}$ is not very large, consequently one can not get correct Higgs mass. 


\begin{tabular}{|c|c|c|c|c|c|c|c|c|c|}
\hline Parameter & $\mathrm{x}=0.5$ & $\mathrm{x}=0.5$ & $\mathrm{x}=0.1$ & $\mathrm{x}=0.1$ & Parameter & $\mathrm{x}=0.5$ & $\mathrm{x}=0.5$ & $\mathrm{x}=0.1$ & $\mathrm{x}=0.1$ \\
\hline$\lambda$ & 0.89 & 0.53 & 0.54 & 0.30 & $y_{N}$ & 0.28 & 0.54 & 0.48 & 0.21 \\
\hline$A_{N}$ & -4301.5 & -1496.9 & -1563.2 & -524.4 & $A_{t}$ & -1768.2 & -992.2 & -1100.1 & -828.1 \\
$m_{R}$ & 3925.9 & 6678.3 & 9942.6 & 7868.5 & $\mu$ & 1395. & 1510. & 249. & 781. \\
$m_{\tilde{\nu}_{1}}$ & 297. & 257. & 333. & 629. & $m_{\tilde{\nu}_{2,3}} / m_{\nu_{2,3}}$ & 3927. & 6679. & 9943. & 7869. \\
\hline$m_{h}$ & 118.93 & 116.84 & 117.10 & 116.53 & $m_{H}$ & 1535. & 1629. & 666. & 1016. \\
$m_{h}^{0+\Delta}$ & 118.92 & 116.80 & 117.08 & 116.53 & $m_{A^{0}}$ & 1543. & 1649. & 676. & 1018. \\
$m_{h}^{0}$ & 116.23 & 116.22 & 116.33 & 116.33 & $m_{H^{ \pm}}$ & 1545. & 1651. & 681. & 1020. \\
\hline$M_{S}$ & 1643. & 1803. & 1724. & 1778. & $m_{\tilde{g}}$ & 2181. & 2186. & 2108. & 2111. \\
$\tilde{t}_{1}$ & 1462. & 1715. & 1604. & 1701. & $\tilde{t}_{2}$ & 1846. & 1896. & 1853. & 1859. \\
$\tilde{b}_{1}$ & 1821. & 1910. & 1863. & 1891. & $\tilde{b}_{2}$ & 1925. & 1925. & 1897. & 1900. \\
$\tilde{\tau}_{1}$ & 259. & 233. & 289. & 313. & $\tilde{\tau}_{2}$ & 301. & 320. & 293. & 316. \\
$\tilde{u}_{1}$ & 1928. & 1930. & 1908. & 1908. & $\tilde{u}_{2}$ & 1942. & 1948. & 1914. & 1930. \\
$\tilde{d}_{1}$ & 1933. & 1925. & 1902. & 1900. & $\tilde{d}_{2}$ & 1934. & 1933. & 1902. & 1911. \\
$\tilde{e}_{1}$ & 301. & 320. & 293. & 316. & $\tilde{e}_{2}$ & 341. & 341. & 338. & 631. \\
$N_{1}$ & 431. & 431. & 236. & 411. & $N_{2}$ & 823. & 821. & 253. & 729. \\
$N_{3}$ & 1403. & 1518. & 422. & 787. & $N_{4}$ & 1408. & 1523. & 807. & 853. \\
$C_{1}$ & 805. & 807. & 243. & 719. & $C_{2}$ & 1399. & 1512. & 790. & 840. \\
\hline
\end{tabular}

Table 7. Benchmark points for Model 6. See caption of table 2 for details of notation.

In table 7, we show four benchmark points, two for $x=0.5$ and two for $x=0.1$. Here $A_{t}$ is less than $A_{N}$. Only for the first benchmark point, Higgs mass is raised by $2 \mathrm{GeV}$ (from MSSM contribution) and others have small $A_{N}$, so there is no correction at all.

\section{7 $\quad$ Model 7}

This model is a Type II model. Here messenger-matter interaction superpotential is $W_{\text {mix }}=$ $\lambda N^{c} H_{d} H_{u}^{m}$. Non-zero corrections to the different soft parameters are shown in eq. (4.10). $\delta M_{H_{d}}^{2}$ and $\delta M_{\tilde{N}^{c}}^{2}$ get both negative one-loop and positive two-loop corrections and other soft masses gets two-loop negative corrections only.

$$
\begin{aligned}
\delta M_{\tilde{Q}}^{2} & =\left[-\frac{3 \alpha_{b} \alpha_{\lambda}}{16 \pi^{2}}\right] \Lambda^{2} \\
\delta M_{\tilde{D}^{c}}^{2} & =\left[-\frac{3 \alpha_{b} \alpha_{\lambda}}{8 \pi^{2}}\right] \Lambda^{2} \\
\delta M_{\tilde{L}}^{2} & =\left[-\frac{3 \alpha_{\lambda}\left(2 \alpha_{N}+\alpha_{\tau}\right)}{16 \pi^{2}}\right] \Lambda^{2} \\
\delta M_{\tilde{E}^{c}}^{2} & =\left[-\frac{3 \alpha_{\lambda} \alpha_{\tau}}{8 \pi^{2}}\right] \Lambda^{2} \\
\delta M_{H_{u}}^{2} & =\left[-\frac{3 \alpha_{N} \alpha_{\lambda}}{8 \pi^{2}}\right] \Lambda^{2}
\end{aligned}
$$




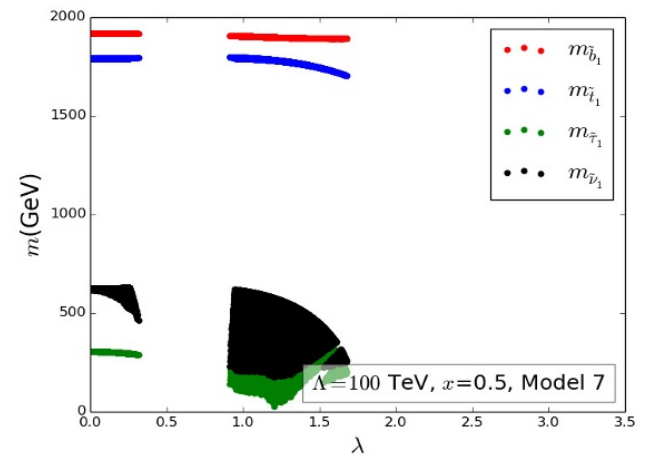

(a)

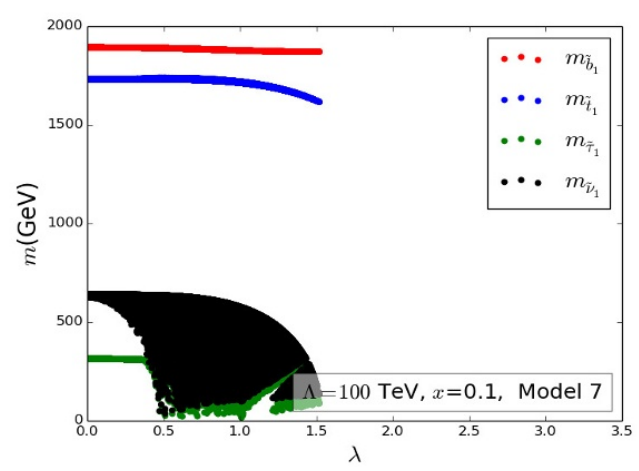

(c)

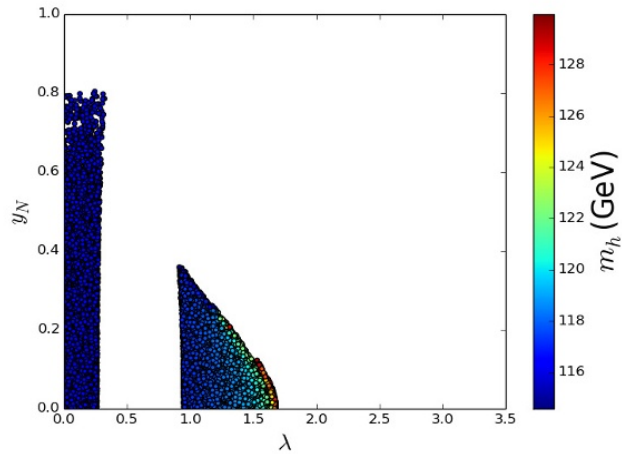

(b)

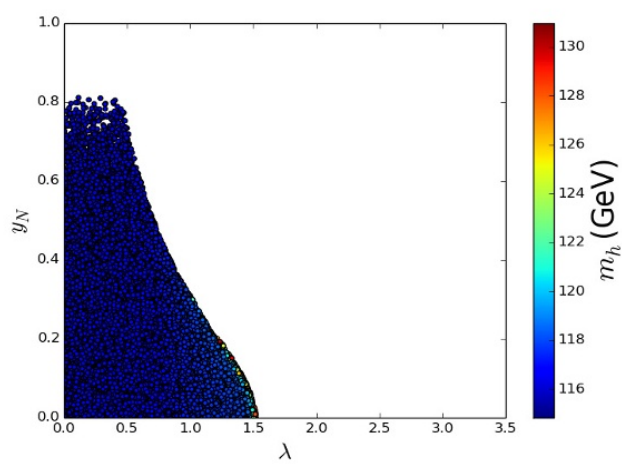

(d)

Figure 7. Model 7: spectrum variation with $\lambda$ and Higgs mass values in $y_{N}$ and $\lambda$ plane. Please see caption of figure 1 for details of notation.

$$
\begin{aligned}
\delta M_{H_{d}}^{2} & =\left[-\frac{\alpha_{\lambda}}{8 \pi} x^{2} h(x)+\frac{3 \alpha_{\lambda}\left(-3 \alpha_{1}-15 \alpha_{2}+10\left(\alpha_{N}+5 \alpha_{\lambda}\right)\right)}{80 \pi^{2}}\right] \Lambda^{2} \\
\delta M_{\tilde{N}^{c}}^{2} & =\left[-\frac{\alpha_{\lambda}}{4 \pi} x^{2} h(x)+\frac{3 \alpha_{\lambda}\left(5\left(-3 \alpha_{2}+3 \alpha_{b}+10 \alpha_{\lambda}+\alpha_{\tau}\right)-3 \alpha_{1}\right)}{40 \pi^{2}}\right] \Lambda^{2} \\
\delta A_{b} & =\left[-\frac{3 \alpha_{\lambda}}{4 \pi}\right] \Lambda \\
\delta A_{\tau} & =\left[-\frac{3 \alpha_{\lambda}}{4 \pi}\right] \Lambda \\
\delta A_{N} & =\left[-\frac{3 \alpha_{\lambda}}{2 \pi}\right] \Lambda .
\end{aligned}
$$

There is no constraint on $H_{d}$ mass from tachyonic mass condition. Thus one may expect large $\lambda$ here. For both the value of $x$, maximum allowed values of $\lambda$ is $\sim 1.5$ because beyond this point lightest stau becomes tachyonic. However, there exists two band of $\lambda$ values for $x=0.5$ because of "non-convergent Higgs spectrum" as can be seen in figure 7(a). One gets a $125 \mathrm{GeV}$ Higgs (figure $7(\mathrm{~b}) \&(\mathrm{~d})$ ) for $\lambda \sim 1.5$ in both cases.

In this model $A_{t}$ term is not generated at the boundary. $A_{N}$ is responsible for generation of correct Higgs mass. One can see from the benchmark points in table 8 that absolute 


\begin{tabular}{|c|c|c|c|c|c|c|c|c|c|}
\hline Parameter & $\mathrm{x}=0.5$ & $\mathrm{x}=0.5$ & $\mathrm{x}=0.1$ & $\mathrm{x}=0.1$ & Parameter & $\mathrm{x}=0.5$ & $\mathrm{x}=0.5$ & $\mathrm{x}=0.1$ & $\mathrm{x}=0.1$ \\
\hline$\lambda$ & 1.60 & 1.34 & 1.26 & 1.43 & $y_{N}$ & 0.07 & 0.17 & 0.17 & 0.09 \\
\hline$A_{N}$ & -9785.4 & -6806.3 & -5988.2 & -7756.1 & $A_{t}$ & -583.6 & -574.8 & -692.3 & -698.4 \\
$m_{R}$ & 6480.5 & 3029.5 & 7172.6 & 7859.8 & $\mu$ & 929. & 858. & 932. & 957. \\
$m_{\tilde{\nu}_{1}}$ & 275. & 281. & 238. & 164. & $m_{\tilde{\nu}_{2,3}} / m_{\nu_{2,3}}$ & 6481. & 3030. & 7173. & 7860. \\
\hline$m_{h}$ & 125.73 & 120.38 & 118.71 & 123.27 & $m_{H}$ & 7172. & 4394. & 5315. & 6890. \\
$m_{h}^{0+\Delta}$ & 125.73 & 120.47 & 118.71 & 123.27 & $m_{A^{0}}$ & 7165. & 4392. & 5316. & 6885. \\
$m_{h}^{0}$ & 116.23 & 116.23 & 116.33 & 116.33 & $m_{H^{ \pm}}$ & 7165. & 4393. & 5317. & 6885. \\
\hline$M_{S}$ & 1779. & 1821. & 1752. & 1717. & $m_{\tilde{g}}$ & 2187. & 2187. & 2111. & 2110. \\
$\tilde{t}_{1}$ & 1723. & 1769. & 1685. & 1646. & $\tilde{t}_{2}$ & 1838. & 1875. & 1821. & 1791. \\
$\tilde{b}_{1}$ & 1891. & 1894. & 1873. & 1870. & $\tilde{b}_{2}$ & 1933. & 1922. & 1906. & 1914. \\
$\tilde{\tau}_{1}$ & 210. & 201. & 202. & 112. & $\tilde{\tau}_{2}$ & 448. & 462. & 522. & 414. \\
$\tilde{u}_{1}$ & 1862. & 1905. & 1858. & 1822. & $\tilde{u}_{2}$ & 1968. & 1966. & 1941. & 1942. \\
$\tilde{d}_{1}$ & 1957. & 1934. & 1925. & 1943. & $\tilde{d}_{2}$ & 2005. & 1980. & 1967. & 1988. \\
$\tilde{e}_{1}$ & 448. & 485. & 522. & 414. & $\tilde{e}_{2}$ & 625. & 485. & 568. & 690. \\
$N_{1}$ & 430. & 430. & 411. & 411. & $N_{2}$ & 804. & 785. & 774. & 777. \\
$N_{3}$ & 938. & 866. & 942. & 967. & $N_{4}$ & 967. & 913. & 964. & 987. \\
$C_{1}$ & 790. & 773. & 760. & 763. & $C_{2}$ & 955. & 901. & 953. & 976. \\
\hline
\end{tabular}

Table 8. Benchmark points for Model 7. See caption of table 2 for details of notation.

values of $A_{N}$ can be $\sim 10 \mathrm{TeV}$ for $x=0.5$. Typical correction to the Higgs mass from its MSSM value $\left(m_{h}^{0}\right)$ is $8 \mathrm{GeV}$. In all the benchmark points, $A_{t}\left(M_{\mathrm{SUSY}}\right)$ is always less than $A_{N}$.

\subsection{Model 8}

This is a Type II model having $W_{\text {mix }}=\frac{1}{2} \lambda N^{c} N^{c} S^{m}$. Note that $S_{m}$ is a gauge singlet. Thus if we consider the messenger sector to be consists of only this field then no usual soft masses will be generated. That is why we consider two sets of messengers: (a) singlet $\left(S_{m}\right)$, which appears in the $W_{\text {mix }}$ and $5 \oplus \overline{5}$, which gives usual GMSB boundary conditions. None of the MSSM fields get one-loop corrections here. However they get two-loop negative corrections as shown in the equation below. The field $\tilde{N}^{c}$ gets both the one-loop and two-loop corrections.

$$
\begin{aligned}
\delta M_{\tilde{L}}^{2} & =\left[-\frac{3 \alpha_{N} \alpha_{\lambda}}{16 \pi^{2}}\right] \Lambda^{2} \\
\delta M_{H_{u}}^{2} & =\left[-\frac{3 \alpha_{N} \alpha_{\lambda}}{16 \pi^{2}}\right] \Lambda^{2} \\
\delta M_{\tilde{N}^{c}}^{2} & =\left[-\frac{\alpha_{\lambda}}{8 \pi} x^{2} h(x)+\frac{3 \alpha_{\lambda}\left(4 \alpha_{N}+13 \alpha_{\lambda}\right)}{32 \pi^{2}}\right] \Lambda^{2} \\
\delta A_{N} & =\left[-\frac{3 \alpha_{\lambda}}{4 \pi}\right] \Lambda .
\end{aligned}
$$




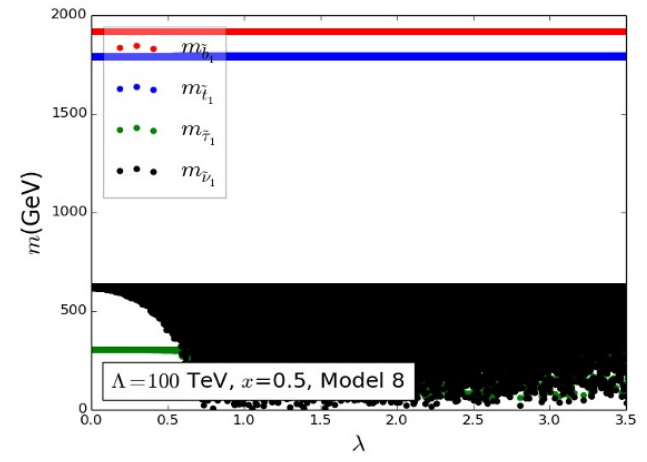

(a)

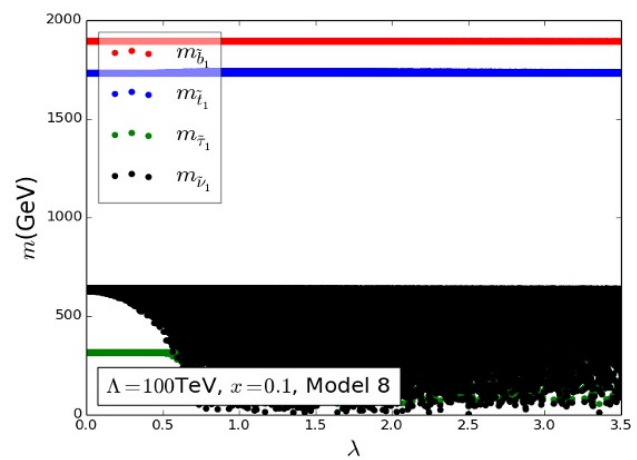

(c)

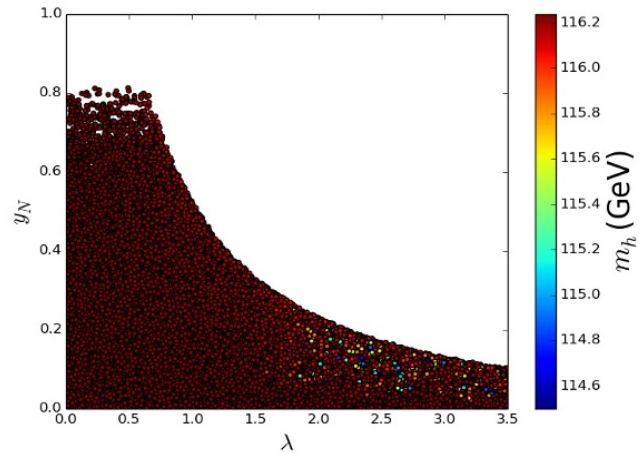

(b)

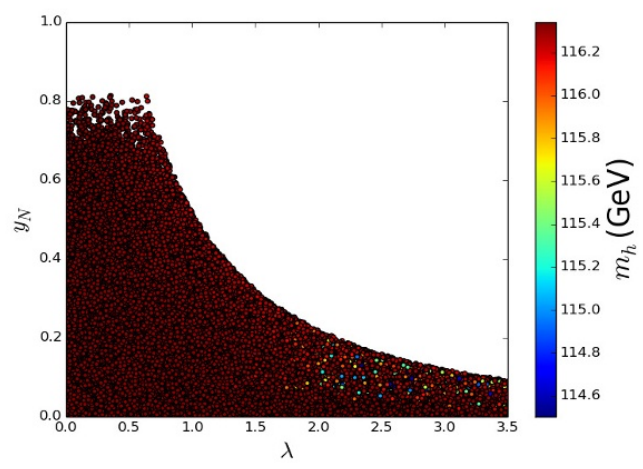

(d)

Figure 8. Model 8: spectrum variation with $\lambda$ and Higgs mass values in $y_{N}$ and $\lambda$ plane. Please see caption of figure 1 for details of notation.

As $\delta M_{\tilde{L}}^{2}$ is negative, $d_{1,2}$ will not be small enough to raise the Higgs mass. That is why we are not getting a $125 \mathrm{GeV}$ Higgs even though very large values of $\lambda$ are allowed (figure 8(a) $\&(\mathrm{c})$ ) and $\sim 10 \mathrm{TeV} A_{N}$ is generated the $M_{\mathrm{SUSY}}$ scale (table 9 ).

In this model no other $A$-terms except the $A_{N}$ is generated at the boundary. As we have discussed earlier only $A_{N}$ is not sufficient. One also need direct coupling of $\tilde{L}$ with the messenger field to get correct Higgs mass.

\subsection{Model 9}

This model is based on the $W_{\text {mix }}=\lambda N^{c} S S_{m}$ interaction. Like the previous model, messenger sector consists of two types of fields for the same reasons: (a) singlet $\left(S_{m}\right)$, which appears in the $W_{\text {mix }}$ and $5 \oplus \overline{5}$, which gives usual GMSB boundary conditions. Soft mass corrections from $W_{\text {mix }}$ are given below:

$$
\begin{aligned}
\delta M_{\tilde{L}}^{2} & =\left[-\frac{3 \alpha_{N} \alpha_{\lambda}}{16 \pi^{2}}\right] \Lambda^{2} \\
\delta M_{H_{u}}^{2} & =\left[-\frac{3 \alpha_{N} \alpha_{\lambda}}{16 \pi^{2}}\right] \Lambda^{2}
\end{aligned}
$$




\begin{tabular}{|c|c|c|c|c|c|c|c|c|c|}
\hline Parameter & $\mathrm{x}=0.5$ & $\mathrm{x}=0.5$ & $\mathrm{x}=0.1$ & $\mathrm{x}=0.1$ & Parameter & $\mathrm{x}=0.5$ & $\mathrm{x}=0.5$ & $\mathrm{x}=0.1$ & $\mathrm{x}=0.1$ \\
\hline$\lambda$ & 2.53 & 0.98 & 2.79 & 1.52 & $y_{N}$ & 0.12 & 0.37 & 0.12 & 0.29 \\
\hline$A_{N}$ & -12137.9 & -1814.3 & -14733.5 & -4307.4 & $A_{t}$ & -576.05 & -575.24 & -689.4 & -682.4 \\
$m_{R}$ & 2374.2 & 5253.4 & 4854.8 & 7984.7 & $\mu$ & 800. & 762. & 926. & 921. \\
$m_{\tilde{\nu}_{1}}$ & 367. & 456. & 261. & 272. & $m_{\tilde{\nu}_{2,3}} / m_{\nu_{2,3}}$ & 2377. & 5254. & 4855. & 7985. \\
\hline$m_{h}$ & 115.47 & 116.20 & 116.23 & 116.27 & $m_{H}$ & 1028. & 989. & 1131. & 1114. \\
$m_{h}^{0+\Delta}$ & 116.21 & 116.19 & 116.27 & 116.25 & $m_{A^{0}}$ & 1026. & 995. & 1136. & 1132. \\
$m_{h}^{0}$ & 116.23 & 116.22 & 116.33 & 116.33 & $m_{H^{ \pm}}$ & 1029. & 998. & 1138. & 1135. \\
\hline$M_{S}$ & 1843. & 1843. & 1798. & 1799. & $m_{\tilde{g}}$ & 2188. & 2188. & 2111. & 2111. \\
$\tilde{t}_{1}$ & 1793. & 1793. & 1738. & 1739. & $\tilde{t}_{2}$ & 1894. & 1894. & 1861. & 1861. \\
$\tilde{b}_{1}$ & 1917. & 1917. & 1892. & 1892. & $\tilde{b}_{2}$ & 1921. & 1921. & 1898. & 1898. \\
$\tilde{\tau}_{1}$ & 299. & 302. & 256. & 261. & $\tilde{\tau}_{2}$ & 306. & 306. & 318. & 318. \\
$\tilde{u}_{1}$ & 1929. & 1929. & 1907. & 1907. & $\tilde{u}_{2}$ & 1969. & 1969. & 1942. & 1942. \\
$\tilde{d}_{1}$ & 1921. & 1921. & 1899. & 1898. & $\tilde{d}_{2}$ & 1955. & 1955. & 1926. & 1926. \\
$\tilde{e}_{1}$ & 306. & 306. & 318. & 318. & $\tilde{e}_{2}$ & 390. & 461. & 330. & 330. \\
$N_{1}$ & 430. & 430. & 412. & 412. & $N_{2}$ & 755. & 730. & 771. & 770. \\
$N_{3}$ & 806. & 767. & 932. & 928. & $N_{4}$ & 880. & 867. & 956. & 952. \\
$C_{1}$ & 746. & 721. & 757. & 757. & $C_{2}$ & 867. & 853. & 946. & 942. \\
\hline
\end{tabular}

Table 9. Benchmark points for Model 8. See caption of table 2 for details of notation.

$$
\begin{aligned}
\delta M_{\tilde{N}^{c}}^{2} & =\left[-\frac{\alpha_{\lambda}}{8 \pi} x^{2} h(x)+\frac{21 \alpha_{\lambda}^{2}}{16 \pi^{2}}\right] \Lambda^{2} \\
\delta M_{\tilde{S}}^{2} & =\left[-\frac{\alpha_{\lambda}}{8 \pi} x^{2} h(x)+\frac{3 \alpha_{\lambda}\left(2 \alpha_{N}+7 \alpha_{\lambda}\right)}{16 \pi^{2}}\right] \Lambda^{2} \\
\delta A_{N} & =\left[-\frac{3 \alpha_{\lambda}}{4 \pi}\right] \Lambda .
\end{aligned}
$$

Here $\delta M_{\tilde{S}}^{2}$ has both the one-loop and two-loop contributions. Note that SUSY preserving mass of $\tilde{S}$ is $\mu_{s}$ which is too small. Secondly, $\tilde{S}$ do not get any mass from gauge mediation as it is SM gauge singlet. Thus lower values of $\lambda$ are ruled out by requirement of non-negative soft mass of $\tilde{S}$ :

$$
\lambda \geq \sqrt{\left(\frac{2}{3} x^{2} h(x) \pi-2 \alpha_{N}\right) \frac{4}{7} \pi .}
$$

Another point to be noted that $\delta M_{\tilde{S}}^{2}$ is RG invariant below the messenger scale as there is no Yukawa or trilinear coupling involving $\tilde{S}$ below that.

Spectrum of this model is similar to the previous model except for the fact that $\lambda$ has now lower limit as shown in figure 9(a) and figure 9(c). These values are $\sim 1$ and $\sim 0.2$ for $x=0.5$ and $x=0.1$ respectively. The squark masses are independent of $\lambda$ as can be seen in figure $9(\mathrm{a})$ and figure $9(\mathrm{c})$. 


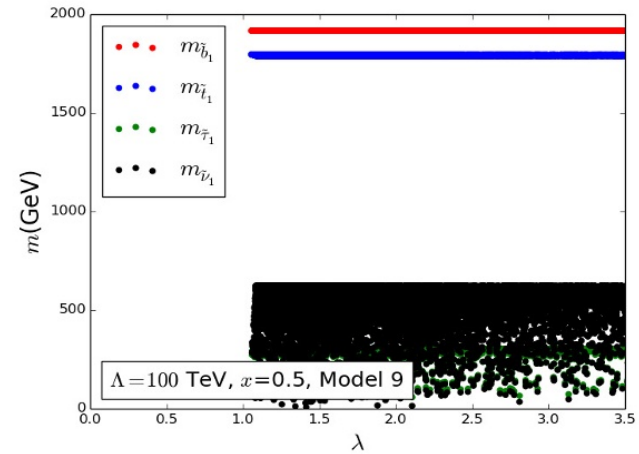

(a)

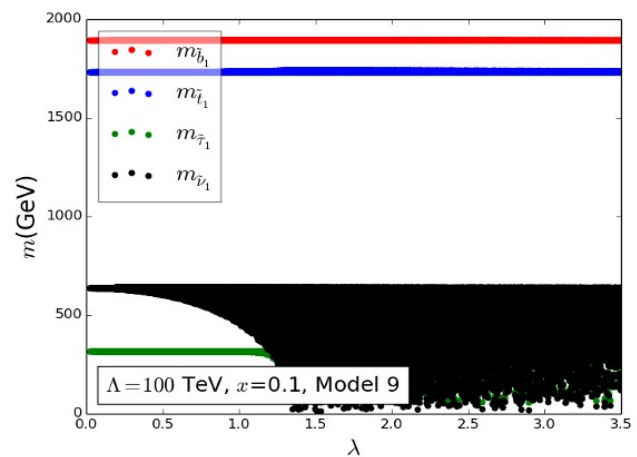

(c)

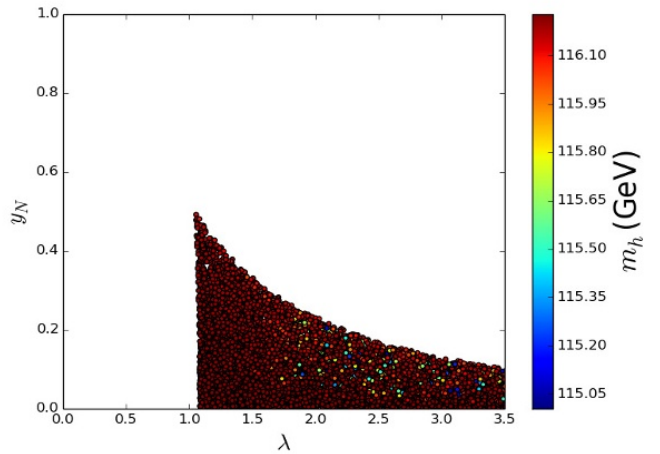

(b)

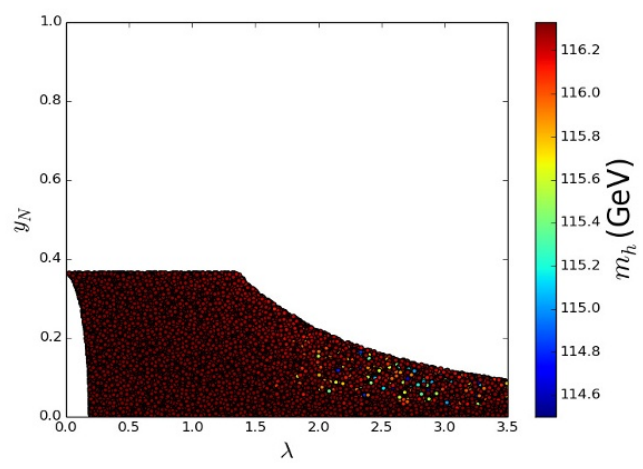

(d)

Figure 9. Model 9: spectrum variation with $\lambda$ and Higgs mass values in $y_{N}$ and $\lambda$ plane. Please see caption of figure 1 for details of notation.

Higgs mass values are given in $\lambda$ vs $y_{N}$ plane. Note that we are getting Higgs mass around $116 \mathrm{GeV}$. Failure of this depicts the importance of heavy slepton. In this model, sleptons are not heavy as they are not getting any messenger-matter correction. Hence $d_{1,2}$ are large and there is no significant increase in the Higgs mass. In this model also only $A_{N}$ is generated at the boundary. In table 10, four benchmark points are shown.

\subsection{Model 10}

This is a Type I model with $W_{\text {mix }}=\lambda N^{c} H_{u}^{m} H_{d}^{m}$. Note that, in this interaction there is only one matter field. Thus choices of the messenger fields are arbitrary. For example, one can have (a) $N^{c} 1_{m} \overline{1}_{m}$, (b) $N^{c} 5_{m} \overline{5}_{m}$, and (c) $N^{c} 10_{m} \overline{10}_{m}$. Here we consider the messenger having representation $5 \oplus \overline{5}$. The corrections to the soft masses for this messenger-matter interaction at the boundary are given in eq. (4.14). The $\delta M_{\tilde{N}^{c}}^{2}$ gets both one-loop negative and two-loop positive corrections. The $\delta M_{\tilde{L}}^{2}$ and $\delta M_{H_{u}}^{2}$ are generated at two-loop level.

$$
\begin{aligned}
\delta M_{\tilde{L}}^{2} & =\left[-\frac{3 \alpha_{N} \alpha_{\lambda}}{8 \pi^{2}}\right] \Lambda^{2} \\
\delta M_{H_{u}}^{2} & =\left[-\frac{3 \alpha_{N} \alpha_{\lambda}}{8 \pi^{2}}\right] \Lambda^{2}
\end{aligned}
$$




\begin{tabular}{|c|c|c|c|c|c|c|c|c|c|}
\hline Parameter & $\mathrm{x}=0.5$ & $\mathrm{x}=0.5$ & $\mathrm{x}=0.1$ & $\mathrm{x}=0.1$ & Parameter & $\mathrm{x}=0.5$ & $\mathrm{x}=0.5$ & $\mathrm{x}=0.1$ & $\mathrm{x}=0.1$ \\
\hline$\lambda$ & 2.40 & 1.49 & 2.26 & 1.08 & $y_{N}$ & 0.10 & 0.22 & 0.17 & 0.36 \\
\hline$A_{N}$ & -10950.2 & -4218.6 & -9702.2 & -2178.6 & $A_{t}$ & -580.8 & -575.3 & -684.6 & -684.3 \\
$m_{R}$ & 5772.3 & 3053.2 & 3746.7 & 4631.7 & $\mu$ & 732. & 763. & 939. & 872. \\
$m_{\tilde{\nu}_{1}}$ & 508. & 452. & 195. & 418. & $m_{\tilde{\nu}_{2,3}} / m_{\nu_{2,3}}$ & 5772. & 3054. & 3748. & 4632. \\
\hline$m_{h}$ & 116.21 & 116.17 & 116.10 & 116.29 & $m_{H}$ & 968. & 996. & 1144. & 1085. \\
$m_{h}^{0+\Delta}$ & 116.21 & 116.19 & 116.25 & 116.27 & $m_{A^{0}}$ & 972. & 996. & 1147. & 1091. \\
$m_{h}^{0}$ & 116.23 & 116.23 & 116.33 & 116.33 & $m_{H^{ \pm}}$ & 975. & 999. & 1149. & 1094. \\
\hline$M_{S}$ & 1842. & 1843. & 1799. & 1798. & $m_{\tilde{g}}$ & 2188. & 2188. & 2111. & 2111. \\
$\tilde{t}_{1}$ & 1792. & 1793. & 1739. & 1737. & $\tilde{t}_{2}$ & 1894. & 1894. & 1861. & 1861. \\
$\tilde{b}_{1}$ & 1917. & 1917. & 1892. & 1892. & $\tilde{b}_{2}$ & 1921. & 1921. & 1898. & 1898. \\
$\tilde{\tau}_{1}$ & 303. & 302. & 202. & 311. & $\tilde{\tau}_{2}$ & 306. & 306. & 318. & 318. \\
$\tilde{u}_{1}$ & 1929. & 1929. & 1907. & 1907. & $\tilde{u}_{2}$ & 1969. & 1969. & 1942. & 1941. \\
$\tilde{d}_{1}$ & 1921. & 1921. & 1898. & 1899. & $\tilde{d}_{2}$ & 1954. & 1955. & 1926. & 1925. \\
$\tilde{e}_{1}$ & 306. & 306. & 318. & 318. & $\tilde{e}_{2}$ & 512. & 459. & 324. & 425. \\
$N_{1}$ & 429. & 430. & 412. & 412. & $N_{2}$ & 707. & 731. & 772. & 761. \\
$N_{3}$ & 737. & 768. & 945. & 878. & $N_{4}$ & 860. & 868. & 967. & 911. \\
$C_{1}$ & 699. & 722. & 759. & 749. & $C_{2}$ & 845. & 853. & 958. & 901. \\
\hline
\end{tabular}

Table 10. Benchmark points for Model 9. See caption of table 2 for details of notation.

$$
\begin{aligned}
\delta M_{\tilde{N}^{c}}^{2} & =\left[-\frac{\alpha_{\lambda}}{2 \pi} x^{2} h(x)+\frac{3 \alpha_{\lambda}\left(-3 \alpha_{1}-15 \alpha_{2}+40 \alpha_{\lambda}\right)}{40 \pi^{2}}\right] \Lambda^{2} \\
\delta A_{N} & =\left[-\frac{3 \alpha_{\lambda}}{2 \pi}\right] \Lambda .
\end{aligned}
$$

Spectral variation with $\lambda$ of this model is similar to models 8 and 9 which is expected from the nature of messenger-matter corrections: (a) all of them get corrections to $M_{\tilde{L}}^{2}, M_{\tilde{N}^{c}}^{2}, M_{H_{u}}^{2}$ and $A_{N}$, and (b) right handed neutrinos are much heavier compared to the messenger-matter corrections at the boundary. Though $\lambda$ can be very large (figure 10(a) \& (c)) and a very large $A_{N}$ is generated at the $M_{\text {SUSY }}$ scale (table 11), Higgs mass obtained can be atmost $117 \mathrm{GeV}$ (figure 10(b) \& (d)). This model also fails like the previous two models for the same reason. The benchmark points are given in table 11.

\subsection{Model 11}

This is a Type II model with the messenger-matter interaction term $W_{\text {mix }}=\lambda L Q D_{m}$. Here messenger fields belong to $5 \oplus \overline{5}$ representation. As both the matter fields are $\mathrm{SU}(2)$ doublets, all the MSSM fields get corrections to their soft masses as well as non-zero trilinear couplings.

$$
\delta M_{\tilde{Q}}^{2}=\left[-\frac{\alpha_{\lambda}}{8 \pi} x^{2} h(x)+\frac{\alpha_{\lambda}\left(5\left(-9 \alpha_{2}-16 \alpha_{3}+3\left(\alpha_{N}+14 \alpha_{\lambda}+\alpha_{\tau}\right)\right)-7 \alpha_{1}\right)}{80 \pi^{2}}\right] \Lambda^{2}
$$




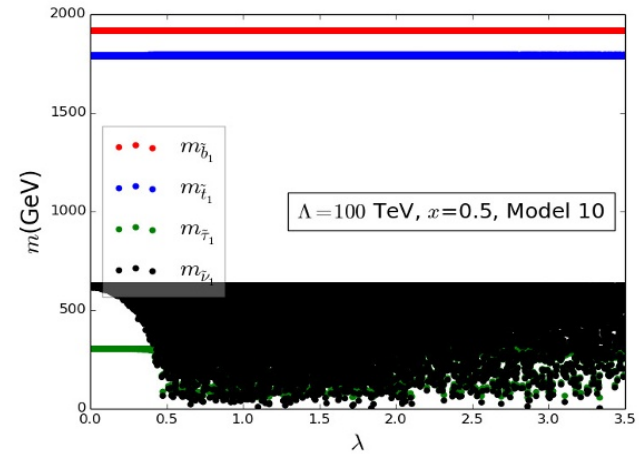

(a)

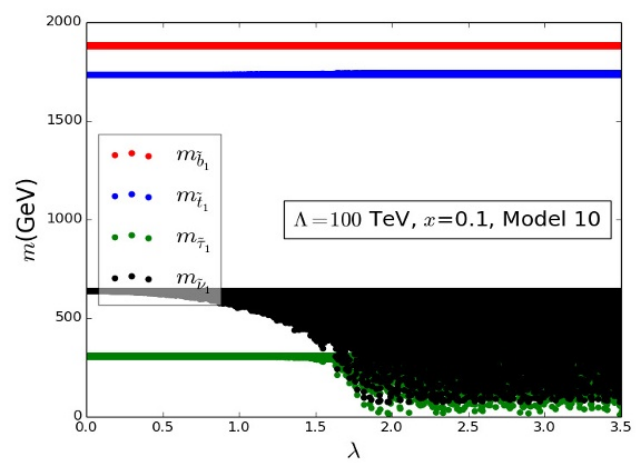

(c)

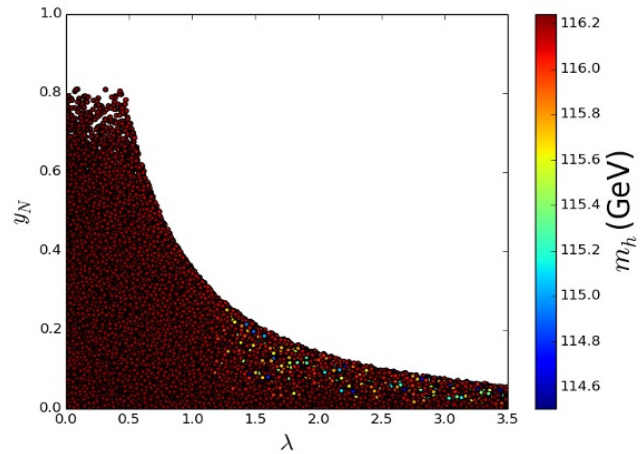

(b)

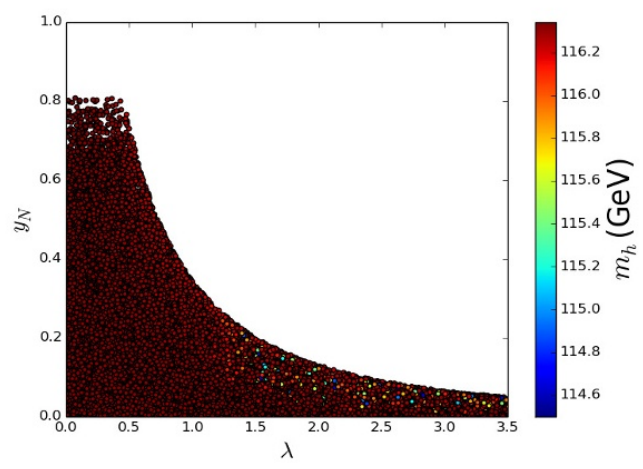

(d)

Figure 10. Model 10: spectrum variation with $\lambda$ and Higgs mass values in $y_{N}$ and $\lambda$ plane. Please see caption of figure 1 for details of notation.

$$
\begin{aligned}
\delta M_{\tilde{U}^{c}}^{2} & =\left[-\frac{3 \alpha_{t} \alpha_{\lambda}}{8 \pi^{2}}\right] \Lambda^{2} \\
\delta M_{\tilde{D}^{c}}^{2} & =\left[-\frac{3 \alpha_{b} \alpha_{\lambda}}{8 \pi^{2}}\right] \Lambda^{2} \\
\delta M_{\tilde{L}}^{2} & =\left[-3 \frac{\alpha_{\lambda}}{8 \pi} x^{2} h(x)+\frac{3 \alpha_{\lambda}\left(5\left(-9 \alpha_{2}-16 \alpha_{3}+3\left(\alpha_{b}+\alpha_{t}+14 \alpha_{\lambda}\right)\right)-7 \alpha_{1}\right)}{80 \pi^{2}}\right] \Lambda^{2} \\
\delta M_{\tilde{E}^{c}}^{2} & =\left[-\frac{9 \alpha_{\lambda} \alpha_{\tau}}{8 \pi^{2}}\right] \Lambda^{2} \\
\delta M_{H_{u}}^{2} & =\left[-\frac{9\left(\alpha_{N}+\alpha_{t}\right) \alpha_{\lambda}}{16 \pi^{2}}\right] \Lambda^{2} \\
\delta M_{H_{d}}^{2}= & {\left[-\frac{9 \alpha_{\lambda}\left(\alpha_{b}+\alpha_{\tau}\right)}{16 \pi^{2}}\right] \Lambda^{2} } \\
\delta M_{\tilde{N}^{c}}^{2} & =\left[-\frac{9 \alpha_{N} \alpha_{\lambda}}{8 \pi^{2}}\right] \Lambda^{2} \\
\delta A_{t} & =\left[-\frac{3 \alpha_{\lambda}}{4 \pi}\right] \Lambda
\end{aligned}
$$




\begin{tabular}{|c|c|c|c|c|c|c|c|c|c|}
\hline Parameter & $\mathrm{x}=0.5$ & $\mathrm{x}=0.5$ & $\mathrm{x}=0.1$ & $\mathrm{x}=0.1$ & Parameter & $\mathrm{x}=0.5$ & $\mathrm{x}=0.5$ & $\mathrm{x}=0.1$ & $\mathrm{x}=0.1$ \\
\hline$\lambda$ & 2.28 & 0.99 & 2.25 & 1.04 & $y_{N}$ & 0.10 & 0.36 & 0.10 & 0.27 \\
\hline$A_{N}$ & -19775.1 & -3696.8 & -19295.2 & -4050.9 & $A_{t}$ & -575.6 & -568.4 & -690.3 & -684.8 \\
$m_{R}$ & 3577.1 & 8253.0 & 6582.5 & 5477.0 & $\mu$ & 861. & 873. & 945. & 889. \\
$m_{\tilde{\nu}_{1}}$ & 164. & 81. & 169. & 374. & $m_{\tilde{\nu}_{2,3}} / m_{\nu_{2,3}}$ & 3579. & 8253. & 6583. & 5477. \\
\hline$m_{h}$ & 115.56 & 116.09 & 116.24 & 116.28 & $m_{H}$ & 1074. & 1062. & 1144. & 1096. \\
$m_{h}^{0+\Delta}$ & 116.17 & 116.07 & 116.26 & 116.27 & $m_{A^{0}}$ & 1076. & 1086. & 1153. & 1105. \\
$m_{h}^{0}$ & 116.23 & 116.23 & 116.33 & 116.33 & $m_{H^{ \pm}}$ & 1079. & 1089. & 1155. & 1108. \\
\hline$M_{S}$ & 1844. & 1845. & 1798. & 1798. & $m_{\tilde{g}}$ & 2188. & 2188. & 2111. & 2111. \\
$\tilde{t}_{1}$ & 1795. & 1796. & 1738. & 1737. & $\tilde{t}_{2}$ & 1894. & 1895. & 1861. & 1861. \\
$\tilde{b}_{1}$ & 1917. & 1917. & 1892. & 1892. & $\tilde{b}_{2}$ & 1921. & 1921. & 1898. & 1898. \\
$\tilde{\tau}_{1}$ & 186. & 81. & 170. & 308. & $\tilde{\tau}_{2}$ & 306. & 306. & 318. & 318. \\
$\tilde{u}_{1}$ & 1929. & 1929. & 1907. & 1907. & $\tilde{u}_{2}$ & 1970. & 1970. & 1942. & 1942. \\
$\tilde{d}_{1}$ & 1921. & 1921. & 1898. & 1899. & $\tilde{d}_{2}$ & 1956. & 1957. & 1926. & 1925. \\
$\tilde{e}_{1}$ & 306. & 306. & 318. & 318. & $\tilde{e}_{2}$ & 312. & 310. & 323. & 386. \\
$N_{1}$ & 430. & 431. & 412. & 412. & $N_{2}$ & 784. & 788. & 773. & 765. \\
$N_{3}$ & 867. & 879. & 952. & 896. & $N_{4}$ & 913. & 921. & 973. & 925. \\
$C_{1}$ & 772. & 776. & 760. & 752. & $C_{2}$ & 901. & 909. & 964. & 915. \\
\hline
\end{tabular}

Table 11. Benchmark points for Model 10. See caption of table 2 for details of notation.

$$
\begin{aligned}
\delta A_{b} & =\left[-\frac{3 \alpha_{\lambda}}{4 \pi}\right] \Lambda \\
\delta A_{\tau} & =\left[-\frac{9 \alpha_{\lambda}}{4 \pi}\right] \Lambda \\
\delta A_{N} & =\left[-\frac{9 \alpha_{\lambda}}{4 \pi}\right] \Lambda .
\end{aligned}
$$

Here $\delta M_{\tilde{L}}^{2}$ gets one-loop negative and two-loop positive contributions. For $x=0.5$, one-loop contribution dominates over two-loop contributions in this model. For very small value of $\lambda(\gtrsim 0.1)$, lightest stau and sneutrino become tachyonic as shown in figure 11(a) and again become positive for $\lambda>1$. On the other hand, for $x=0.1$, two-loop negative contribution to $M_{\tilde{E}^{c}}^{2}$ is responsible for smallness of $\lambda$. Here lightest stau and sneutrino become tachyonic for $\lambda \gtrsim 0.15$ and large $\lambda$ region (0.6-1.1) provides positive spectrum. In this model, all the A-terms are generated at the boundary. We can see from the (figure 11(b) and figure 11(d), the upper allowed band of $\lambda$ in both $x=0.5$ and $x=0.1$ case can provide correct Higgs mass. In table 12, four benchmark points for this models are shown. One can see from table 12 that the combination of both matter-messenger and neutrino-sneutrino corrections will reproduce correct Higgs mass and one may get tachyonic spectrum including only the matter-messenger boundary conditions. 


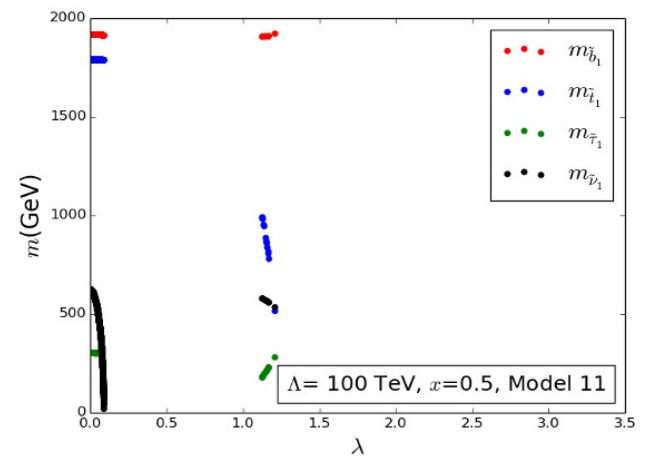

(a)

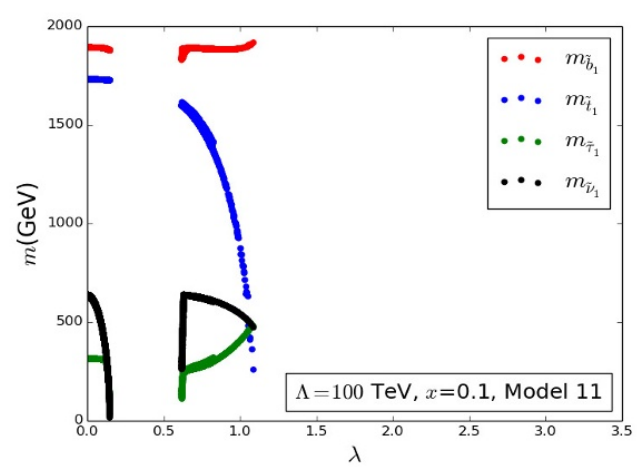

(c)

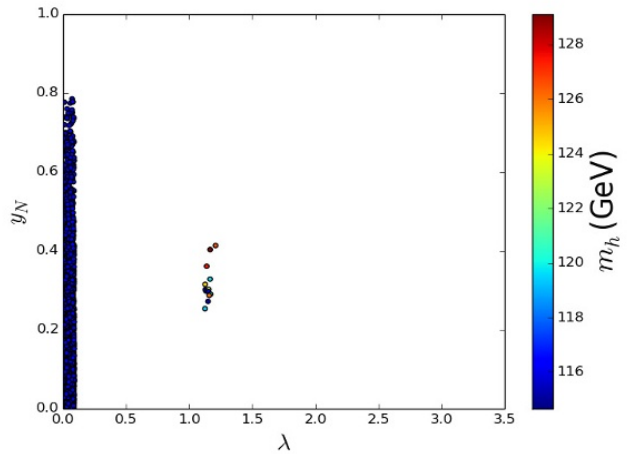

(b)

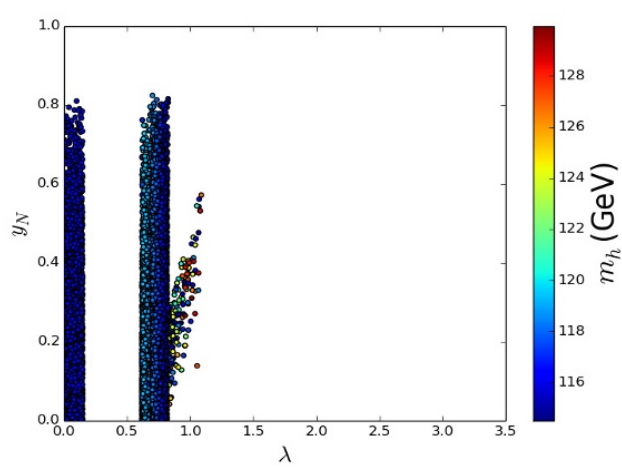

(d)

Figure 11. Model 11: spectrum variation with $\lambda$ and Higgs mass values in $y_{N}$ and $\lambda$ plane. Please see caption of figure 1 for details of notations.

\subsection{Model 12}

This model represents the messenger-matter interaction superpotential $W_{\text {mix }}=\lambda L D^{c} Q_{m}$. Here messenger fields transform as $10 \oplus \overline{10}$. One-loop and two-loop corrections due to this interaction are shown below:

$$
\begin{aligned}
\delta M_{\tilde{Q}}^{2} & =\left[-\frac{\alpha_{b} \alpha_{\lambda}}{8 \pi^{2}}\right] \Lambda^{2} \\
\delta M_{\tilde{D}^{c}}^{2} & =\left[-\frac{\alpha_{\lambda}}{12 \pi} x^{2} h(x)+\frac{\alpha_{\lambda}\left(-7 \alpha_{1}+5\left(-9 \alpha_{2}-16 \alpha_{3}+3 \alpha_{N}+18 \alpha_{\lambda}+3 \alpha_{\tau}\right)\right)}{120 \pi^{2}}\right] \Lambda^{2} \\
\delta M_{\tilde{L}}^{2} & =\left[-\frac{\alpha_{\lambda}}{8 \pi} x^{2} h(x)+\frac{\alpha_{\lambda}\left(-7 \alpha_{1}-45 \alpha_{2}-80 \alpha_{3}+30 \alpha_{b}+90 \alpha_{\lambda}\right)}{80 \pi^{2}}\right] \Lambda^{2} \\
\delta M_{\tilde{E}^{c}}^{2} & =\left[-\frac{3 \alpha_{\lambda} \alpha_{\tau}}{8 \pi^{2}}\right] \Lambda^{2} \\
\delta M_{\tilde{N}^{c}}^{2} & =\left[-\frac{3 \alpha_{N} \alpha_{\lambda}}{8 \pi^{2}}\right] \Lambda^{2} \\
\delta M_{H_{u}}^{2} & =\left[-\frac{3 \alpha_{N} \alpha_{\lambda}}{16 \pi^{2}}\right] \Lambda^{2}
\end{aligned}
$$




\begin{tabular}{|c|c|c|c|c|c|c|c|c|c|}
\hline Parameter & $\mathrm{x}=0.5$ & $\mathrm{x}=0.5$ & $\mathrm{x}=0.1$ & $\mathrm{x}=0.1$ & Parameter & $\mathrm{x}=0.5$ & $\mathrm{x}=0.5$ & $\mathrm{x}=0.1$ & $\mathrm{x}=0.1$ \\
\hline$\lambda$ & 1.16 & 1.13 & 0.81 & 0.74 & $y_{N}$ & 0.29 & 0.32 & 0.11 & 0.14 \\
\hline$A_{N}$ & -7366.9 & -6930.3 & -3616.7 & -3008.0 & $A_{t}$ & -2601.8 & -2484.2 & -1624.7 & -1472.0 \\
$m_{R}$ & 5154.4 & 4568.6 & 3015.6 & 2158.0 & $\mu$ & 2157. & 2095. & 1465. & 1331. \\
$m_{\tilde{\nu}_{1}}$ & 4947. & 4290. & 2982. & 2113. & $m_{\tilde{\nu}_{2,3}} / m_{\nu_{2,3}}$ & 5161. & 4576. & 3022. & 2169. \\
\hline$m_{h}$ & 126.14 & 124.72 & 125.66 & 122.15 & $m_{H}$ & 2153. & 2105. & 1576. & 1463. \\
$m_{h}^{0+\Delta}$ & --- & --- & 114.25 & 117.34 & $m_{A^{0}}$ & 2200. & 2148. & 1579. & 1464. \\
$m_{h}^{0}$ & 116.23 & 116.23 & 116.33 & 116.33 & $m_{H^{ \pm}}$ & 2197. & 2146. & 1580. & 1466. \\
\hline$M_{S}$ & 1264. & 1376. & 1613. & 1673. & $m_{\tilde{g}}$ & 2203. & 2200. & 2117. & 2114. \\
$\tilde{t}_{1}$ & 838. & 991. & 1390. & 1491. & $\tilde{t}_{2}$ & 1908. & 1909. & 1872. & 1876. \\
$\tilde{b}_{1}$ & 1909. & 1907. & 1885. & 1888. & $\tilde{b}_{2}$ & 1943. & 1936. & 1903. & 1902. \\
$\tilde{\tau}_{1}$ & 217. & 182. & 294. & 272. & $\tilde{\tau}_{2}$ & 4958. & 4300. & 2993. & 2131. \\
$\tilde{u}_{1}$ & 1916. & 1918. & 1895. & 1903. & $\tilde{u}_{2}$ & 2027. & 2022. & 1996. & 1997. \\
$\tilde{d}_{1}$ & 1943. & 1937. & 1903. & 1903. & $\tilde{d}_{2}$ & 2028. & 2024. & 1997. & 1999. \\
$\tilde{e}_{1}$ & 477. & 441. & 402. & 361. & $\tilde{e}_{2}$ & 570. & 584. & 617. & 630. \\
$N_{1}$ & 431. & 431. & 412. & 412. & $N_{2}$ & 834. & 832. & 788. & 787. \\
$N_{3}$ & 2148. & 2087. & 1473. & 1339. & $N_{4}$ & 2150. & 2090. & 1478. & 1345. \\
$C_{1}$ & 807. & 807. & 770. & 770. & $C_{2}$ & 2156. & 2095. & 1468. & 1335. \\
\hline
\end{tabular}

Table 12. Benchmark points for Model 11. See caption of table 2 for details of notation.

$$
\begin{aligned}
\delta M_{H_{d}}^{2} & =\left[-\frac{3 \alpha_{\lambda}\left(2 \alpha_{b}+\alpha_{\tau}\right)}{16 \pi^{2}}\right] \Lambda^{2} \\
\delta A_{b} & =\left[-\frac{\alpha_{\lambda}}{2 \pi}\right] \Lambda \\
\delta A_{\tau} & =\left[-\frac{3 \alpha_{\lambda}}{4 \pi}\right] \Lambda \\
\delta A_{N} & =\left[-\frac{3 \alpha_{\lambda}}{4 \pi}\right] \Lambda .
\end{aligned}
$$

Here $\delta M_{\tilde{D}^{c}}^{2}$ and $\delta M_{\tilde{L}}^{2}$ get both the one-loop negative and two-loop positive contributions. For $x=0.5$, cancellation between one-loop and two-loop contribution to $M_{\tilde{L}}^{2}$ is severe. We can see from figure 12(a) and figure 12(c), two band of $\lambda$ values are allowed in both $x=0.5$ and $x=0.1$ case.

Here all the A-terms, except $A_{t}$, are generated. In larger $\lambda$ region, we can achieve a $125 \mathrm{GeV}$ Higgs. In the table 13, four benchmark points are shown for this model. Like model 11, in this model also, for $x=0.5$ case, only the combination of matter-messenger mixing and neutrino-sneutrino corrections can provide positive spectrum and one may get tachyonic spectrum considering only the matter messenger mixing boundary conditions. 


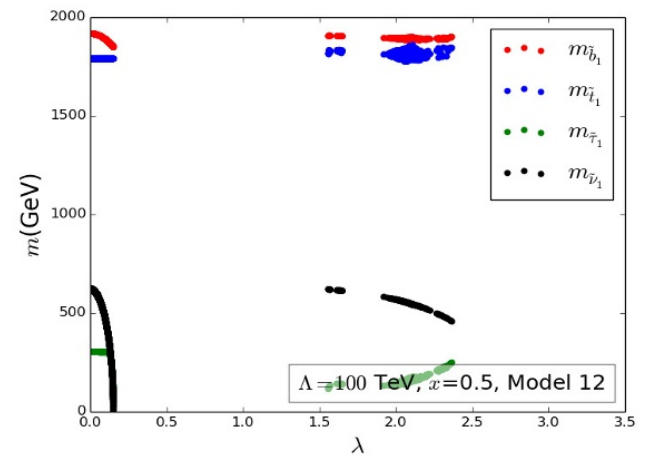

(a)

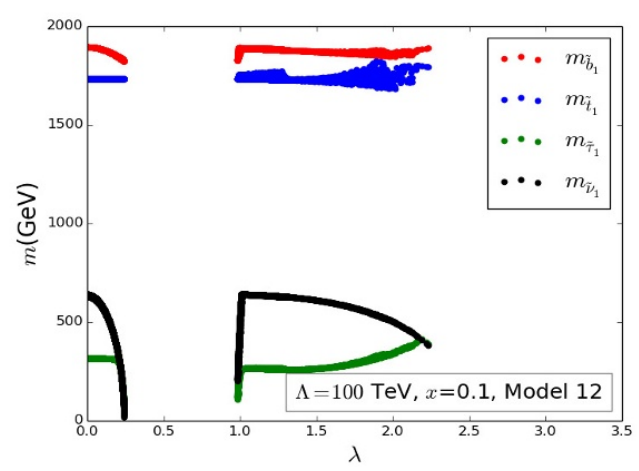

(c)

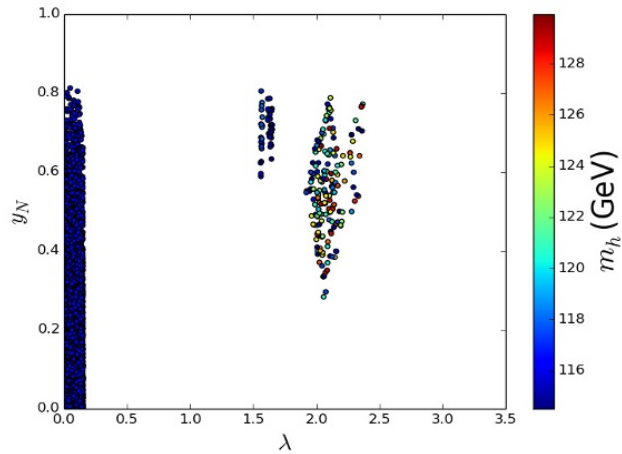

(b)

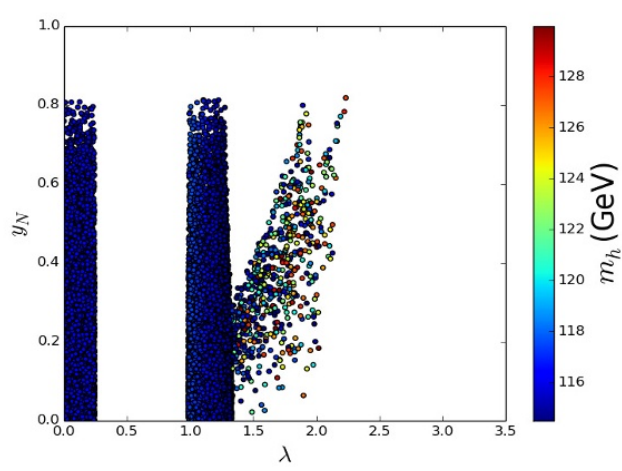

(d)

Figure 12. Model 12: spectrum variation with $\lambda$ and Higgs mass values in $y_{N}$ and $\lambda$ plane. Please see caption of figure 1 for details of notation.

\subsection{Model 13}

Model 13 is Type II model having $W_{\text {mix }}=\lambda L E^{c} H_{d}^{m}$ and soft mass corrections are listed below:

$$
\begin{aligned}
\delta M_{\tilde{L}}^{2} & =\left[-\frac{\alpha_{\lambda}}{8 \pi} x^{2} h(x)+\frac{3 \alpha_{\lambda}\left(-9 \alpha_{1}-15 \alpha_{2}+10\left(5 \alpha_{\lambda}+\alpha_{\tau}\right)\right)}{80 \pi^{2}}\right] \Lambda^{2} \\
\delta M_{\tilde{E}^{c}}^{2} & =\left[-\frac{\alpha_{\lambda}}{4 \pi} x^{2} h(x)+\frac{3 \alpha_{\lambda}\left(5\left(-3 \alpha_{2}+\alpha_{N}+10 \alpha_{\lambda}+2 \alpha_{\tau}\right)-9 \alpha_{1}\right)}{40 \pi^{2}}\right] \Lambda^{2} \\
\delta M_{H_{u}}^{2} & =\left[-\frac{3 \alpha_{N} \alpha_{\lambda}}{16 \pi^{2}}\right] \Lambda^{2} \\
\delta M_{H_{d}}^{2} & =\left[-\frac{9 \alpha_{\lambda} \alpha_{\tau}}{16 \pi^{2}}\right] \Lambda^{2} \\
\delta M_{\tilde{N}^{c}}^{2} & =\left[-\frac{3 \alpha_{N} \alpha_{\lambda}}{8 \pi^{2}}\right] \Lambda^{2}
\end{aligned}
$$




\begin{tabular}{|c|c|c|c|c|c|c|c|c|c|}
\hline Parameter & $\mathrm{x}=0.5$ & $\mathrm{x}=0.5$ & $\mathrm{x}=0.1$ & $\mathrm{x}=0.1$ & Parameter & $\mathrm{x}=0.5$ & $\mathrm{x}=0.5$ & $\mathrm{x}=0.1$ & $\mathrm{x}=0.1$ \\
\hline$\lambda$ & 2.11 & 1.96 & 1.55 & 1.34 & $y_{N}$ & 0.50 & 0.49 & 0.20 & 0.24 \\
\hline$A_{N}$ & -8138.8 & -7044.0 & -4541.8 & -3381.1 & $A_{t}$ & -501.2 & -514.9 & -686.7 & -685.3 \\
$m_{R}$ & 7297.3 & 7115.6 & 5011.5 & 3108.0 & $\mu$ & 1726. & 1520. & 854. & 860. \\
$m_{\tilde{\nu}_{1}}$ & 8098. & 6325. & 4904. & 3219. & $m_{\tilde{\nu}_{2,3}} / m_{\nu_{2,3}}$ & 7295. & 7119. & 5014. & 3103. \\
\hline$m_{h}$ & 124.62 & 124.33 & 125.54 & 124.33 & $m_{H}$ & 1536. & 1414. & 952. & 1009. \\
$m_{h}^{0+\Delta}$ & --- & --- & 109.21 & 113.62 & $m_{A^{0}}$ & 1726. & 1552. & 964. & 1017. \\
$m_{h}^{0}$ & 116.23 & 116.23 & 116.33 & 116.33 & $m_{H^{ \pm}}$ & 1719. & 1548. & 962. & 1018. \\
\hline$M_{S}$ & 1836. & 1841. & 1781. & 1790. & $m_{\tilde{g}}$ & 2206. & 2203. & 2123. & 2120. \\
$\tilde{t}_{1}$ & 1801. & 1802. & 1719. & 1729. & $\tilde{t}_{2}$ & 1871. & 1881. & 1845. & 1853. \\
$\tilde{b}_{1}$ & 1889. & 1892. & 1867. & 1876. & $\tilde{b}_{2}$ & 1932. & 1927. & 1904. & 1900. \\
$\tilde{\tau}_{1}$ & 158. & 132. & 265. & 259. & $\tilde{\tau}_{2}$ & 8093. & 6330. & 4910. & 3210. \\
$\tilde{u}_{1}$ & 1893. & 1906. & 1890. & 1899. & $\tilde{u}_{2}$ & 1965. & 1965. & 1932. & 1936. \\
$\tilde{d}_{1}$ & 1992. & 1984. & 1947. & 1939. & $\tilde{d}_{2}$ & 2016. & 2014. & 1998. & 1997. \\
$\tilde{e}_{1}$ & 520. & 450. & 431. & 370. & $\tilde{e}_{2}$ & 550. & 581. & 609. & 629. \\
$N_{1}$ & 432. & 432. & 412. & 412. & $N_{2}$ & 824. & 823. & 759. & 760. \\
$N_{3}$ & 1733. & 1529. & 860. & 866. & $N_{4}$ & 1737. & 1534. & 898. & 902. \\
$C_{1}$ & 805. & 805. & 744. & 746. & $C_{2}$ & 1727. & 1523. & 886. & 891. \\
\hline
\end{tabular}

Table 13. Benchmark points for Model 12. See caption of table 2 for details of notation.

$$
\begin{aligned}
\delta A_{\tau} & =\left[-\frac{9 \alpha_{\lambda}}{4 \pi}\right] \Lambda^{2} \\
\delta A_{N} & =\left[-\frac{3 \alpha_{\lambda}}{4 \pi}\right] \Lambda .
\end{aligned}
$$

Here both $\delta M_{\tilde{L}}^{2}$ and $\delta M_{\tilde{E}^{c}}^{2}$ get one-loop negative correction and two-loop positive correction. As earlier, the interplay between the positive and negative contributions plays an important role. Parameter space for $\lambda$ is broken down into two parts. In the region where $\lambda$ is small, one-loop contributions dominate over the two-loop contributions. Just above $\lambda \sim 0.1$ $(\sim 0.2)$, lightest stau becomes tachyonic for $x=0.5(0.1)$ as shown in figure 13(a) and figure 13(c). After certain values of $\lambda$, the two-loop contributions dominate over the oneloop contributions. Note that, the gap between two regions of parameter spaces of $\lambda$ is larger for $x=0.5$ which is expected. Higgs mass values are shown in figure 13(b) and figure $13(\mathrm{~d})$. In the second region where $\lambda$ is higher, a $125 \mathrm{GeV}$ Higgs is achievable.

Here at the boundary, $A_{\tau}$ and $A_{N}$ are generated. In table 14, four benchmark points are shown for both $x=0.5$ and 0.1 case, the absolute values of $A_{N}$ at the $M_{\text {SUSY }}$ scale is $\gtrsim 1.5 \mathrm{TeV}$. The Higgs mass is raised by $4-10 \mathrm{GeV}$ in these points (table 14 ). 


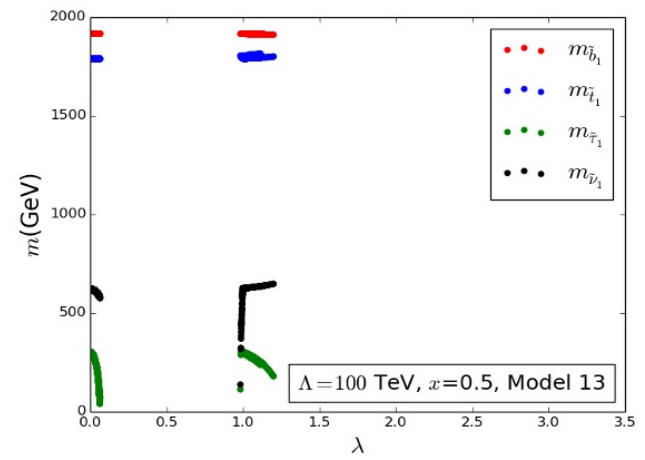

(a)

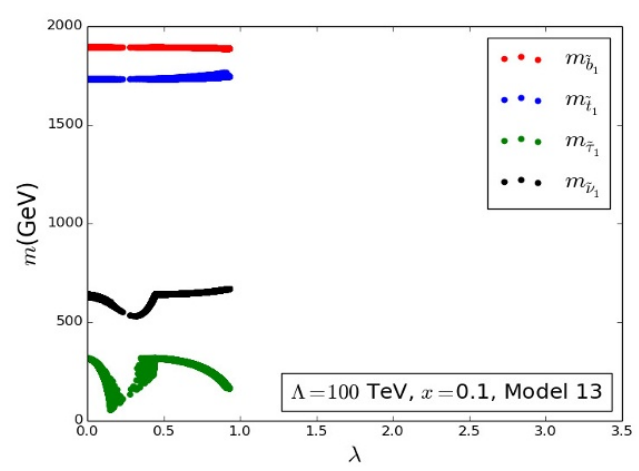

(c)

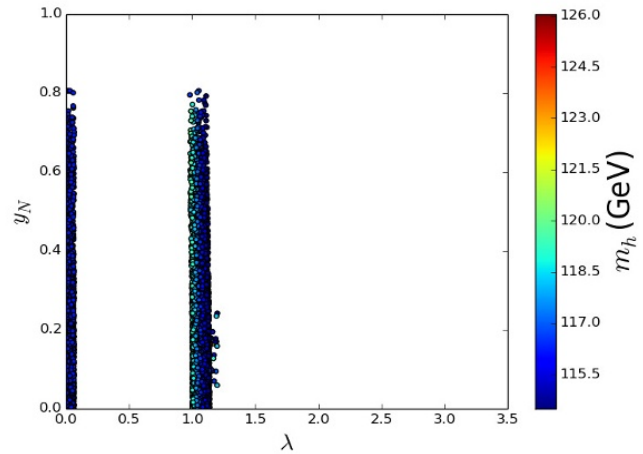

(b)

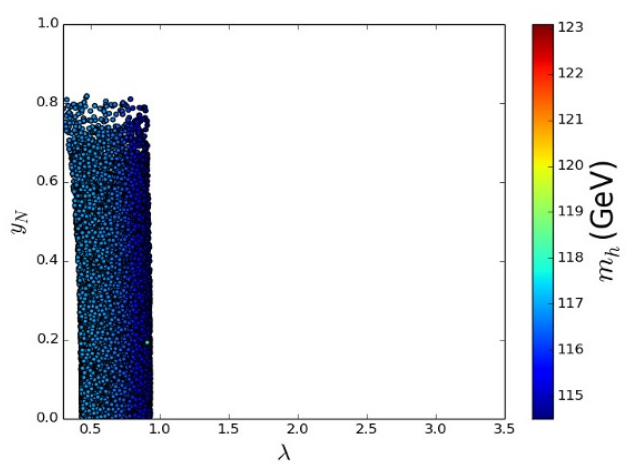

(d)

Figure 13. Model 13: spectrum variation with $\lambda$ and Higgs mass values in $y_{N}$ and $\lambda$ plane. Note that origins of two right panel plots are 1.0 and 0.3 respectively. Please see caption of figure 1 for details of notation.

\subsection{Model 14}

This model has the following messenger-matter interaction superpotential, $W_{\text {mix }}=$ $\lambda L H_{d} E_{m}^{c}$. The boundary conditions generated for this superpotential are shown in eq. (4.18). All the scalar fields of MSSM except $\tilde{U}^{c}$ get these corrections. Both $\delta M_{\tilde{L}}^{2}$ and $\delta M_{H_{d}}^{2}$ get one-loop negative and the two-loop positive contributions. Rest of the fields get only two-loop negative corrections.

$$
\begin{aligned}
\delta M_{\tilde{Q}}^{2} & =\left[-\frac{\alpha_{b} \alpha_{\lambda}}{16 \pi^{2}}\right] \Lambda^{2} \\
\delta M_{\tilde{D}^{c}}^{2} & =\left[-\frac{\alpha_{b} \alpha_{\lambda}}{8 \pi^{2}}\right] \Lambda^{2} \\
\delta M_{\tilde{L}}^{2} & =\left[-\frac{\alpha_{\lambda}}{24 \pi} x^{2} h(x)+\frac{\alpha_{\lambda}\left(-9 \alpha_{1}+5\left(-3 \alpha_{2}+3 \alpha_{b}+4\left(\alpha_{\lambda}+\alpha_{\tau}\right)\right)\right)}{80 \pi^{2}}\right] \Lambda^{2} \\
\delta M_{\tilde{E}^{c}}^{2} & =\left[-\frac{\alpha_{\lambda} \alpha_{\tau}}{4 \pi^{2}}\right] \Lambda^{2}
\end{aligned}
$$




\begin{tabular}{|c|c|c|c|c|c|c|c|c|c|}
\hline Parameter & $\mathrm{x}=0.5$ & $\mathrm{x}=0.5$ & $\mathrm{x}=0.1$ & $\mathrm{x}=0.1$ & Parameter & $\mathrm{x}=0.5$ & $\mathrm{x}=0.5$ & $\mathrm{x}=0.1$ & $\mathrm{x}=0.1$ \\
\hline$\lambda$ & 1.14 & 0.99 & 0.82 & 0.89 & $y_{N}$ & 0.17 & 0.57 & 0.10 & 0.05 \\
\hline$A_{N}$ & -2493.9 & -1808.9 & -1294.8 & -1517.2 & $A_{t}$ & -581.6 & -564.2 & -700.1 & -700.7 \\
$m_{R}$ & 2326.9 & 9630.6 & 2054.6 & 2437.1 & $\mu$ & 700. & 915. & 764. & 763. \\
$m_{\tilde{\nu}_{1}}$ & 2284. & 556. & 2023. & 2450. & $m_{\tilde{\nu}_{2,3}} / m_{\nu_{2,3}}$ & 2340. & 9631. & 2063. & 2434. \\
\hline$m_{h}$ & 126.02 & 119.55 & 123.18 & 119.50 & $m_{H}$ & 911. & 1066. & 992. & 991. \\
$m_{h}^{0+\Delta}$ & 113.72 & 119.5 & 115.46 & 114.98 & $m_{A^{0}}$ & 914. & 1104. & 993. & 992. \\
$m_{h}^{0}$ & 116.23 & 116.22 & 116.33 & 116.33 & $m_{H^{ \pm}}$ & 916. & 1107. & 996. & 994. \\
\hline$M_{S}$ & 1847. & 1847. & 1800. & 1802. & $m_{\tilde{g}}$ & 2188. & 2188. & 2111. & 2111. \\
$\tilde{t}_{1}$ & 1797. & 1799. & 1738. & 1741. & $\tilde{t}_{2}$ & 1898. & 1896. & 1864. & 1866. \\
$\tilde{b}_{1}$ & 1914. & 1916. & 1889. & 1888. & $\tilde{b}_{2}$ & 1918. & 1921. & 1895. & 1893. \\
$\tilde{\tau}_{1}$ & 2307. & 300. & 2051. & 2439. & $\tilde{\tau}_{2}$ & 3163. & 937. & 2763. & 3327. \\
$\tilde{u}_{1}$ & 1936. & 1929. & 1914. & 1917. & $\tilde{u}_{2}$ & 1967. & 1971. & 1939. & 1938. \\
$\tilde{d}_{1}$ & 1918. & 1921. & 1895. & 1893. & $\tilde{d}_{2}$ & 1952. & 1957. & 1920. & 1919. \\
$\tilde{e}_{1}$ & 644. & 536. & 659. & 666. & $\tilde{e}_{2}$ & 644. & 630. & 659. & 666. \\
$N_{1}$ & 430. & 430. & 412. & 412. & $N_{2}$ & 681. & 797. & 720. & 719. \\
$N_{3}$ & 705. & 921. & 769. & 769. & $N_{4}$ & 856. & 952. & 845. & 845. \\
$C_{1}$ & 673. & 785. & 710. & 710. & $C_{2}$ & 839. & 941. & 831. & 831. \\
\hline
\end{tabular}

Table 14. Benchmark points for Model 13. See caption of table 2 for details of notation.

$$
\begin{aligned}
\delta M_{\tilde{N}^{c}}^{2} & =\left[-\frac{\alpha_{N} \alpha_{\lambda}}{8 \pi^{2}}\right] \Lambda^{2} \\
\delta M_{H_{u}}^{2} & =\left[-\frac{\alpha_{N} \alpha_{\lambda}}{16 \pi^{2}}\right] \Lambda^{2} \\
\delta M_{H_{d}}^{2} & =\left[-\frac{\alpha_{\lambda}}{24 \pi} x^{2} h(x)+\frac{\alpha_{\lambda}\left(-9 \alpha_{1}+5\left(-3 \alpha_{2}+\alpha_{N}+4\left(\alpha_{\lambda}+\alpha_{\tau}\right)\right)\right)}{80 \pi^{2}}\right] \Lambda^{2} \\
A_{b} & =\left[-\frac{\alpha_{\lambda}}{4 \pi}\right] \Lambda \\
A_{\tau} & =\left[-\frac{\alpha_{\lambda}}{2 \pi}\right] \Lambda \\
A_{N} & =\left[-\frac{\alpha_{\lambda}}{4 \pi}\right] \Lambda .
\end{aligned}
$$

We get two band of allowed values of $\lambda$ for $x=0.5$. Note that the one loop and two loop corrections are proportional to $\alpha_{\lambda}$ and $\alpha_{\lambda}^{2}$ respectively. For the smaller values of $\lambda$, one-loop effect dominates over the two loop contribution and as a consequence lightest stau becomes tachyonic beyond $\lambda \sim 0.32$ as shown in figure 14(a). For larger values of $\lambda$, situation gets reversed. However, $\lambda$ cannot be arbitrary large because otherwise $M_{H_{d}}^{2}$ will become very heavy and radiative electroweak symmetry breaking would not be possible. For the other case, where $x=0.1$, one-loop effect is not so strong and the upper limit of $\lambda$ is around $\sim 2.3$ (figure $14(\mathrm{c})$ ). 


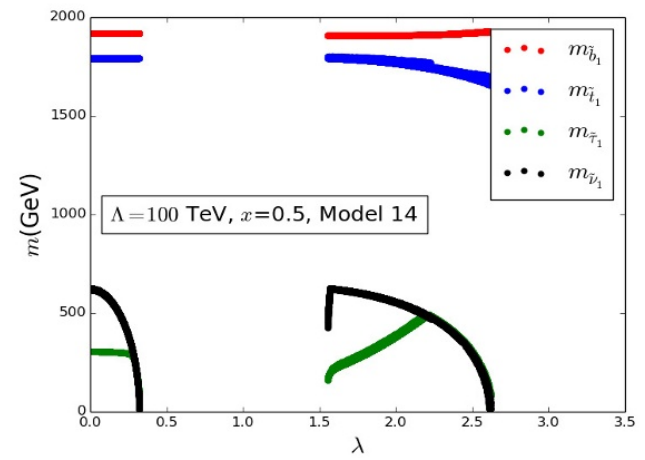

(a)

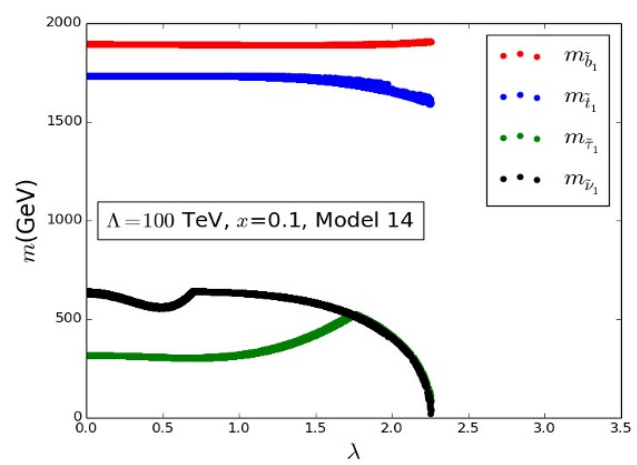

(c)

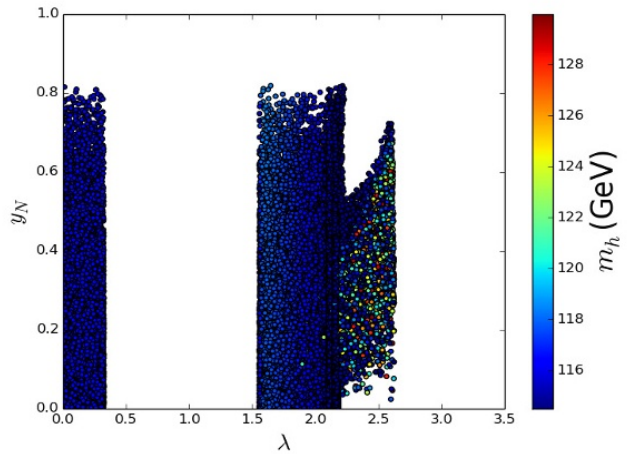

(b)

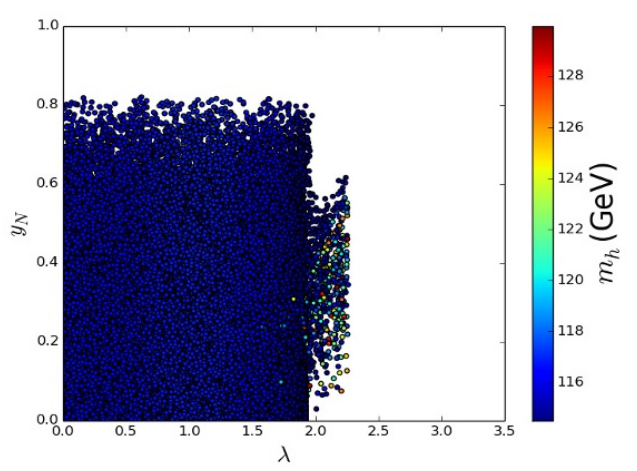

(d)

Figure 14. Model 14: spectrum variation with $\lambda$ and Higgs mass values in $y_{N}$ and $\lambda$ plane. Please see caption of figure 1 for details of notation.

In figure 14(b) and figure 14(d), Higgs mass values are shown in the $\lambda$ vs $y_{N}$ plane. For both the values of $x$, a $125 \mathrm{GeV}$ Higgs is achievable. In table 15 four benchmark points are shown. In this model, no $A_{t}$ term is generated at the boundary. Therefore heavy sleptons $\left(\tilde{\tau}_{2}\right)$ and $A_{N}$ are responsible for giving correct Higgs mass.

\subsection{Model 15}

This is a Type II model based on $5 \oplus \overline{5}$ and $S_{m}$ messenger fields like the model 8 and 9 . The soft mass corrections based on the interaction $\lambda L H_{u} S_{m}$ are given by

$$
\begin{aligned}
\delta M_{\tilde{Q}}^{2} & =\left[-\frac{3 \alpha_{t} \alpha_{\lambda}}{16 \pi^{2}}\right] \Lambda^{2} \\
\delta M_{\tilde{U}^{c}}^{2} & =\left[-\frac{3 \alpha_{t} \alpha_{\lambda}}{8 \pi^{2}}\right] \Lambda^{2} \\
\delta M_{\tilde{L}}^{2} & =\left[-\frac{\alpha_{\lambda}}{8 \pi} x^{2} h(x)+\frac{3 \alpha_{\lambda}\left(5\left(-3 \alpha_{2}+4 \alpha_{N}+3 \alpha_{t}+8 \alpha_{\lambda}\right)-3 \alpha_{1}\right)}{80 \pi^{2}}\right] \Lambda^{2} \\
\delta M_{\tilde{e}^{c}}^{2} & =\left[-\frac{3 \alpha_{\lambda} \alpha_{\tau}}{8 \pi^{2}}\right] \Lambda^{2}
\end{aligned}
$$




\begin{tabular}{|c|c|c|c|c|c|c|c|c|c|}
\hline Parameter & $\mathrm{x}=0.5$ & $\mathrm{x}=0.5$ & $\mathrm{x}=0.1$ & $\mathrm{x}=0.1$ & Parameter & $\mathrm{x}=0.5$ & $\mathrm{x}=0.5$ & $\mathrm{x}=0.1$ & $\mathrm{x}=0.1$ \\
\hline$\lambda$ & 2.16 & 1.82 & 2.16 & 1.59 & $y_{N}$ & 0.30 & 0.18 & 0.37 & 0.17 \\
\hline$A_{N}$ & -2926.0 & -2108.4 & -2875.0 & -1624.1 & $A_{t}$ & -573.2 & -581.1 & -680.4 & -697.9 \\
$m_{R}$ & 3907.0 & 2220.9 & 5876.2 & 2981.9 & $\mu$ & 859. & 693. & 1077. & 777. \\
$m_{\tilde{\nu}_{1}}$ & 4071. & 2176. & 5549. & 2935. & $m_{\tilde{\nu}_{2,3}} / m_{\nu_{2,3}}$ & 3905. & 2229. & 5877. & 2984. \\
\hline$m_{h}$ & 125.39 & 120.65 & 124.87 & 120.93 & $m_{H}$ & 4198. & 2308. & 5734. & 3058. \\
$m_{h}^{0+\Delta}$ & 114.01 & 116.73 & 110.76 & 115.918 & $m_{A^{0}}$ & 4202. & 2308. & 5740. & 3058. \\
$m_{h}^{0}$ & 116.23 & 116.23 & 116.33 & 116.33 & $m_{H^{ \pm}}$ & 4202. & 2310. & 5740. & 3059. \\
\hline$M_{S}$ & 1803. & 1831. & 1692. & 1767. & $m_{\tilde{g}}$ & 2188. & 2188. & 2111. & 2111. \\
$\tilde{t}_{1}$ & 1750. & 1779. & 1623. & 1702. & $\tilde{t}_{2}$ & 1858. & 1884. & 1763. & 1836. \\
$\tilde{b}_{1}$ & 1908. & 1905. & 1900. & 1887. & $\tilde{b}_{2}$ & 1934. & 1922. & 1938. & 1907. \\
$\tilde{\tau}_{1}$ & 461. & 295. & 283. & 451. & $\tilde{\tau}_{2}$ & 4064. & 2192. & 5553. & 2941. \\
$\tilde{u}_{1}$ & 1884. & 1917. & 1789. & 1876. & $\tilde{u}_{2}$ & 1971. & 1966. & 1957. & 1941. \\
$\tilde{d}_{1}$ & 1945. & 1928. & 1958. & 1915. & $\tilde{d}_{2}$ & 1983. & 1966. & 1993. & 1945. \\
$\tilde{e}_{1}$ & 513. & 406. & 749. & 528. & $\tilde{e}_{2}$ & 595. & 600. & 876. & 569. \\
$N_{1}$ & 430. & 429. & 412. & 411. & $N_{2}$ & 786. & 676. & 787. & 729. \\
$N_{3}$ & 867. & 699. & 1088. & 785. & $N_{4}$ & 915. & 856. & 1100. & 854. \\
$C_{1}$ & 772. & 668. & 768. & 718. & $C_{2}$ & 901. & 839. & 1089. & 839. \\
\hline
\end{tabular}

Table 15. Benchmark points for Model 14. See caption of table 2 for details of notation.

$$
\begin{aligned}
\delta M_{H_{u}}^{2} & =\left[-\frac{\alpha_{\lambda}}{8 \pi} x^{2} h(x)+\frac{3 \alpha_{\lambda}\left(5\left(-3 \alpha_{2}+4 \alpha_{N}+8 \alpha_{\lambda}+\alpha_{\tau}\right)-3 \alpha_{1}\right)}{80 \pi^{2}}\right] \Lambda^{2} \\
\delta M_{H_{d}}^{2} & =\left[-\frac{3 \alpha_{\lambda} \alpha_{\tau}}{16 \pi^{2}}\right] \Lambda^{2} \\
\delta M_{\tilde{N}^{c}}^{2} & =\left[-\frac{3 \alpha_{N} \alpha_{\lambda}}{4 \pi^{2}}\right] \Lambda^{2} \\
\delta A_{t} & =\left[-\frac{3 \alpha_{\lambda}}{4 \pi}\right] \Lambda \\
\delta A_{\tau} & =\left[-\frac{3 \alpha_{\lambda}}{4 \pi}\right] \Lambda \\
\delta A_{N} & =\left[-\frac{3 \alpha_{\lambda}}{2 \pi}\right] \Lambda .
\end{aligned}
$$

Here all the MSSM fields, except $D^{c}$, get these messenger-matter corrections. Both the $\delta M_{\tilde{L}}^{2}$ and $\delta M_{H_{u}}^{2}$ get one-loop and two-loop corrections. Like all the models where $\delta M_{\tilde{L}}^{2}$ gets one-loop negative contribution, this model is also a constrained model. One again, $\lambda$ has two allowed regions for $x=0.5$. Maximum value of $\lambda$ in the first band is $\sim 0.2$ as shown in figure 15(a) and that value for the upper band is $\sim 1.2$ which is set by radiative electroweak breaking condition. For $x=0.1$, there is only one band. The maximum value of $\lambda$ is $\sim 0.6$ and this upper limit of $\lambda$ is set by EWSB condition because $M_{H_{u}}^{2}$ gets positive correction whereas squarks get negative contributions at the boundary. 


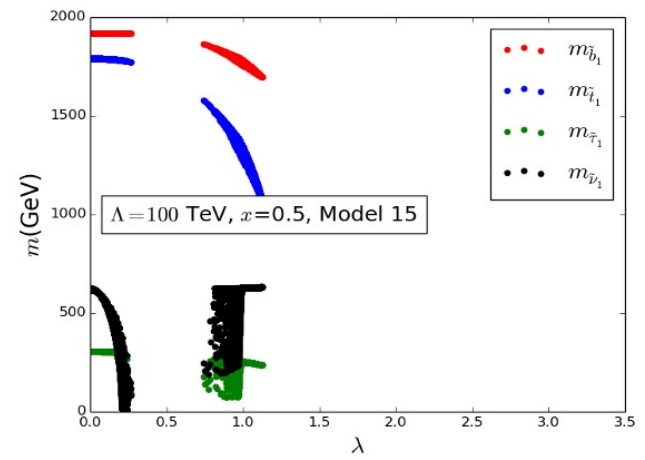

(a)

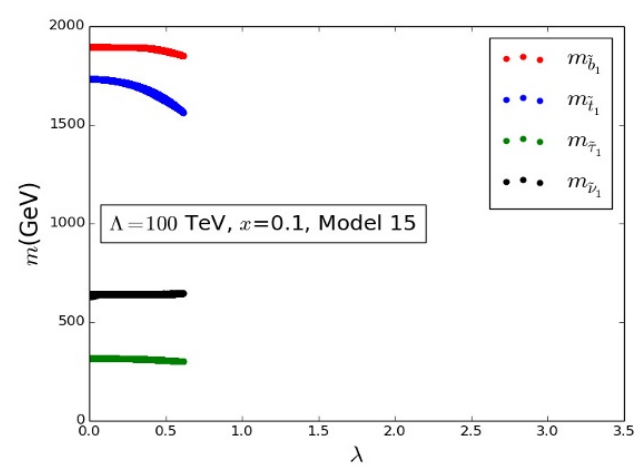

(c)

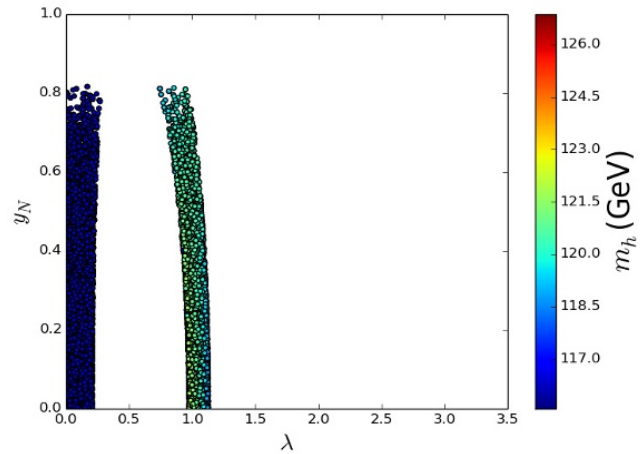

(b)

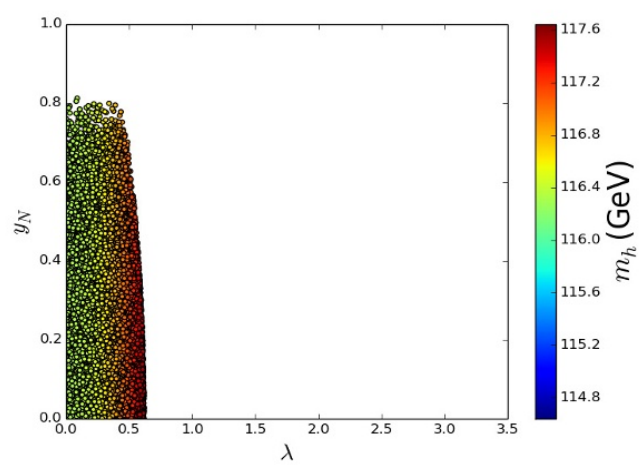

(d)

Figure 15. Model 15: spectrum variation with $\lambda$ and Higgs mass values in $y_{N}$ and $\lambda$ plane. Please see caption of figure 1 for details of notation.

In the right panel on figure 15, Higgs mass values are shown. One cannot get correct Higgs mass for the case of $x=0.1$ because $\lambda$ is too small. In the other case, one can achieve it for the larger values of $\lambda$ (figure 15(b)).

Four benchmark points of the model are shown in table 16. In first benchmark point, we have $125 \mathrm{GeV}$ Higgs mass. We see that a $10 \mathrm{GeV}$ rise in the Higgs mass from the MSSM value.

\subsection{Model 16}

This is a Type II model with $W_{\text {mix }}=\lambda L S H_{u}^{m}$. Corrections due to this superpotential to the soft mass are shown below. None of the squarks gets a correction. Both $\delta M_{\tilde{L}}^{2}$ and $\delta M_{\tilde{S}}^{2}$ get negative one-loop and positive two-loop corrections.

$$
\begin{aligned}
\delta M_{\tilde{L}}^{2} & =\left[-\frac{\alpha_{\lambda}}{8 \pi} x^{2} h(x)+\frac{3 \alpha_{\lambda}\left(-3 \alpha_{1}-15 \alpha_{2}+50 \alpha_{\lambda}\right)}{80 \pi^{2}}\right] \Lambda^{2} \\
\delta M_{\tilde{e}^{c}}^{2} & =\left[-\frac{3 \alpha_{\lambda} \alpha_{\tau}}{8 \pi^{2}}\right] \Lambda^{2}
\end{aligned}
$$




\begin{tabular}{|c|c|c|c|c|c|c|c|c|c|}
\hline Parameter & $\mathrm{x}=0.5$ & $\mathrm{x}=0.5$ & $\mathrm{x}=0.1$ & $\mathrm{x}=0.1$ & Parameter & $\mathrm{x}=0.5$ & $\mathrm{x}=0.5$ & $\mathrm{x}=0.1$ & $\mathrm{x}=0.1$ \\
\hline$\lambda$ & 1.10 & 0.82 & 0.60 & 0.47 & $y_{N}$ & 0.09 & 0.73 & 0.11 & 0.13 \\
\hline$A_{N}$ & -4367.3 & -2275.9 & -1290.1 & -802.4 & $A_{t}$ & -2429.9 & -1561.6 & -1206.5 & -1012.3 \\
$m_{R}$ & 1748.7 & 9648.0 & 2062.1 & 1640.4 & $\mu$ & 806. & 1383. & 326. & 714. \\
$m_{\tilde{\nu}_{1}}$ & 1707. & 468. & 1284. & 945. & $m_{\tilde{\nu}_{2,3}} / m_{\nu_{2,3}}$ & 1767. & 9649. & 2062. & 1641. \\
\hline$m_{h}$ & 126.01 & 119.80 & 117.47 & 117.05 & $m_{H}$ & 1016. & 1440. & 706. & 962. \\
$m_{h}^{0+\Delta}$ & 119.16 & 119.60 & 117.47 & 117.05 & $m_{A^{0}}$ & 1018. & 1529. & 706. & 962. \\
$m_{h}^{0}$ & 116.23 & 116.23 & 116.33 & 116.33 & $m_{H^{ \pm}}$ & 1020. & 1531. & 710. & 965. \\
\hline$M_{S}$ & 1416. & 1683. & 1706. & 1749. & $m_{\tilde{g}}$ & 2178. & 2182. & 2107. & 2109. \\
$\tilde{t}_{1}$ & 1144. & 1521. & 1576. & 1647. & $\tilde{t}_{2}$ & 1752. & 1863. & 1848. & 1857. \\
$\tilde{b}_{1}$ & 1716. & 1842. & 1854. & 1880. & $\tilde{b}_{2}$ & 1928. & 1925. & 1899. & 1900. \\
$\tilde{\tau}_{1}$ & 239. & 252. & 300. & 306. & $\tilde{\tau}_{2}$ & 1729. & 628. & 1285. & 947. \\
$\tilde{u}_{1}$ & 1942. & 1927. & 1903. & 1910. & $\tilde{u}_{2}$ & 1955. & 1939. & 1913. & 1916. \\
$\tilde{d}_{1}$ & 1948. & 1931. & 1904. & 1902. & $\tilde{d}_{2}$ & 1948. & 1932. & 1904. & 1902. \\
$\tilde{e}_{1}$ & 307. & 306. & 318. & 318. & $\tilde{e}_{2}$ & 631. & 485. & 646. & 644. \\
$N_{1}$ & 429. & 431. & 308. & 410. & $N_{2}$ & 761. & 822. & 331. & 685. \\
$N_{3}$ & 812. & 1391. & 426. & 719. & $N_{4}$ & 887. & 1396. & 807. & 829. \\
$C_{1}$ & 748. & 805. & 319. & 677. & $C_{2}$ & 869. & 1387. & 790. & 814. \\
\hline
\end{tabular}

Table 16. Benchmark points for Model 15. See caption of table 2 for details of notation.

$$
\begin{aligned}
\delta M_{H_{u}}^{2} & =\left[-\frac{3 \alpha_{N} \alpha_{\lambda}}{16 \pi^{2}}\right] \Lambda^{2} \\
\delta M_{H_{d}}^{2} & =\left[-\frac{3 \alpha_{\lambda} \alpha_{\tau}}{16 \pi^{2}}\right] \Lambda^{2} \\
\delta M_{\tilde{N}^{c}}^{2} & =\left[-\frac{3 \alpha_{N} \alpha_{\lambda}}{8 \pi^{2}}\right] \Lambda^{2} \\
\delta M_{\tilde{S}}^{2} & =\left[-\frac{\alpha_{\lambda}}{4 \pi} x^{2} h(x)+\frac{3 \alpha_{\lambda}\left(5\left(-3 \alpha_{2}+\alpha_{N}+10 \alpha_{\lambda}+\alpha_{\tau}\right)-3 \alpha_{1}\right)}{40 \pi^{2}}\right] \Lambda^{2} \\
\delta A_{\tau} & =\left[-\frac{3 \alpha_{\lambda}}{4 \pi}\right] \Lambda \\
\delta A_{N} & =\left[-\frac{3 \alpha_{\lambda}}{4 \pi}\right] \Lambda
\end{aligned}
$$

Like the model 9, lower values of $\lambda$ of this model are also ruled out by requirement of non-zero soft mass of $\tilde{S}$ :

$$
\lambda \geq \sqrt{\left(\frac{10}{3} x^{2} h(x) \pi+3 \alpha_{1}+15 \alpha_{2}-5 \alpha_{N}-5 \alpha_{\tau}\right) \frac{2 \pi}{25}},
$$

where minimum value of $\lambda$ has to be $\sim 0.34$ and 0.98 for $x=0.1$ and $x=0.5$ respectively. The sparticle eigenvalues are shown in figure 16(a) and figure 16(c). 


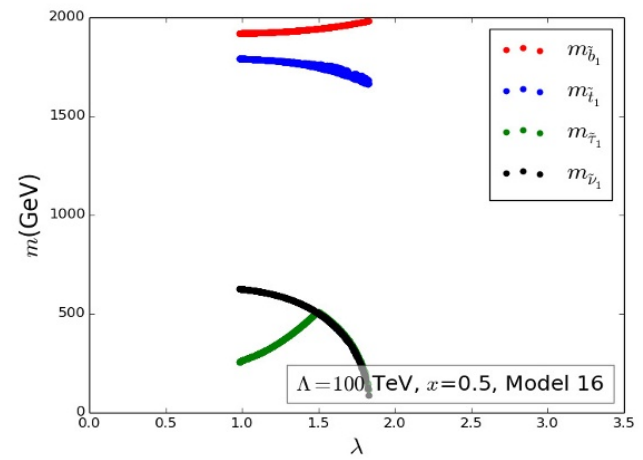

(a)

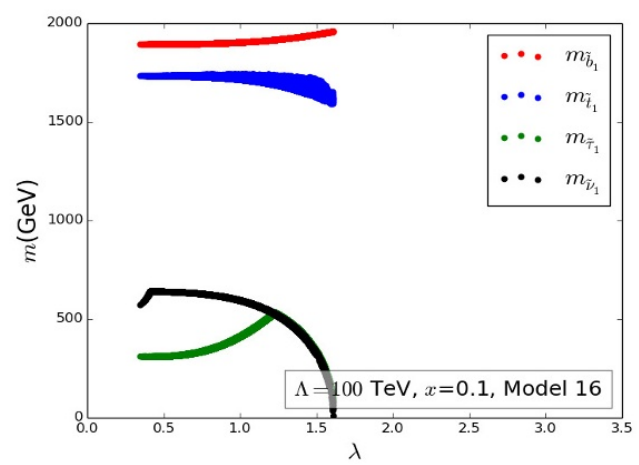

(c)

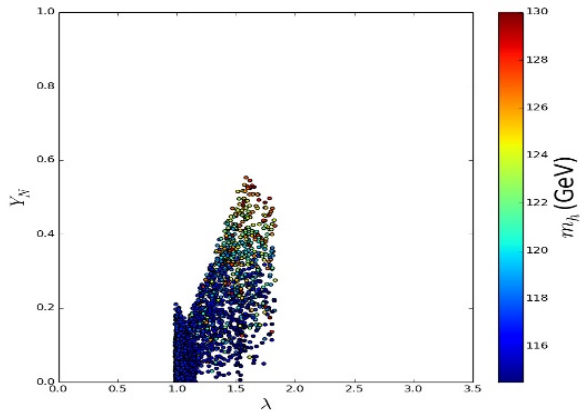

(b)

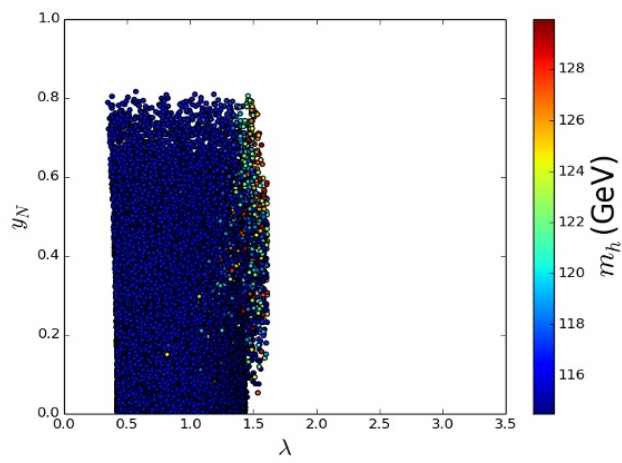

(d)

Figure 16. Model 16: spectrum variation with $\lambda$ and Higgs mass values in $y_{N}$ and $\lambda$ plane. Please see caption of figure 1 for details of notation.

Like other models, in table 17 , benchmark points, both for $x=0.5$ and $x=0.1$ case, are shown. We can see from this table and figure 16(b) and figure 16(d) that required Higgs mass can be achieved in this model. There is a $8 \mathrm{GeV}$ increase in the Higgs mass from its pure MSSM values for all the benchmark points. We can see that all the points have heavy sleptons $\left(\tilde{\tau}_{2}\right)$.

\subsection{Model 17}

This model belongs to Type I category having interaction superpotential, $\lambda L H_{u}^{m} S^{m}$. The one-loop and two-loop corrections are given below:

$$
\begin{aligned}
\delta M_{\tilde{L}}^{2} & =\left[-\frac{\alpha_{\lambda}}{4 \pi} x^{2} h(x)+\frac{9 \alpha_{\lambda}\left(-\alpha_{1}-5 \alpha_{2}+10 \alpha_{\lambda}\right)}{80 \pi^{2}}\right] \Lambda^{2} \\
\delta M_{\tilde{e}^{c}}^{2} & =\left[-\frac{3 \alpha_{\lambda} \alpha_{\tau}}{8 \pi^{2}}\right] \Lambda^{2} \\
\delta M_{H_{u}}^{2} & =\left[-\frac{3 \alpha_{N} \alpha_{\lambda}}{16 \pi^{2}}\right] \Lambda^{2}
\end{aligned}
$$




\begin{tabular}{|c|c|c|c|c|c|c|c|c|c|}
\hline Parameter & $\mathrm{x}=0.5$ & $\mathrm{x}=0.5$ & $\mathrm{x}=0.1$ & $\mathrm{x}=0.1$ & Parameter & $\mathrm{x}=0.5$ & $\mathrm{x}=0.5$ & $\mathrm{x}=0.1$ & $\mathrm{x}=0.1$ \\
\hline$\lambda$ & 1.47 & 1.25 & 1.48 & 0.96 & $y_{N}$ & 0.34 & 0.24 & 0.25 & 0.23 \\
\hline$A_{N}$ & -4031.4 & -2944.9 & -4153.3 & -1767.2 & $A_{t}$ & -567.3 & -577.7 & -690.6 & -695.7 \\
$m_{R}$ & 5683.3 & 3311.6 & 7557.4 & 3021.5 & $\mu$ & 907. & 727. & 838. & 776. \\
$m_{\tilde{\nu}_{1}}$ & 5544. & 3368. & 7325. & 2955. & $m_{\tilde{\nu}_{2,3}} / m_{\nu_{2,3}}$ & 5687. & 3305. & 7558. & 3024. \\
\hline$m_{h}$ & 125.86 & 124.33 & 125.30 & 124.08 & $m_{H}$ & 1012. & 924. & 880. & 978. \\
$m_{h}^{0+\Delta}$ & 114.07 & 115.72 & 113.15 & 115.98 & $m_{A^{0}}$ & 1042. & 931. & 902. & 981. \\
$m_{h}^{0}$ & 116.23 & 116.23 & 116.33 & 116.33 & $m_{H^{ \pm}}$ & 1041. & 932. & 899. & 984. \\
\hline$M_{S}$ & 1809. & 1829. & 1706. & 1782. & $m_{\tilde{g}}$ & 2188. & 2188. & 2111. & 2111. \\
$\tilde{t}_{1}$ & 1757. & 1778. & 1636. & 1718. & $\tilde{t}_{2}$ & 1862. & 1883. & 1778. & 1848. \\
$\tilde{b}_{1}$ & 1938. & 1925. & 1939. & 1900. & $\tilde{b}_{2}$ & 1943. & 1929. & 1950. & 1907. \\
$\tilde{\tau}_{1}$ & 488. & 354. & 358. & 392. & $\tilde{\tau}_{2}$ & 5547. & 3362. & 7327. & 2964. \\
$\tilde{u}_{1}$ & 1887. & 1914. & 1806. & 1892. & $\tilde{u}_{2}$ & 1979. & 1971. & 1963. & 1943. \\
$\tilde{d}_{1}$ & 1943. & 1929. & 1950. & 1907. & $\tilde{d}_{2}$ & 1969. & 1958. & 1957. & 1928. \\
$\tilde{e}_{1}$ & 522. & 425. & 723. & 437. & $\tilde{e}_{2}$ & 580. & 593. & 820. & 607. \\
$N_{1}$ & 430. & 429. & 411. & 411. & $N_{2}$ & 798. & 704. & 756. & 727. \\
$N_{3}$ & 913. & 732. & 843. & 782. & $N_{4}$ & 947. & 861. & 887. & 851. \\
$C_{1}$ & 782. & 695. & 740. & 716. & $C_{2}$ & 935. & 843. & 874. & 837. \\
\hline
\end{tabular}

Table 17. Benchmark points for Model 16. See caption of table 2 for details of notation.

$$
\begin{aligned}
\delta M_{H_{d}}^{2} & =\left[-\frac{3 \alpha_{\lambda} \alpha_{\tau}}{16 \pi^{2}}\right] \Lambda^{2} \\
\delta M_{\tilde{N}^{c}}^{2} & =\left[-\frac{3 \alpha_{N} \alpha_{\lambda}}{8 \pi^{2}}\right] \Lambda^{2} \\
\delta A_{\tau} & =\left[-\frac{3 \alpha_{\lambda}}{4 \pi}\right] \Lambda \\
\delta A_{N} & =\left[-\frac{3 \alpha_{\lambda}}{4 \pi}\right] \Lambda
\end{aligned}
$$

Because of one-loop negative contribution to $\delta M_{\tilde{L}}^{2}$, lightest stau and sneutrino become tachyonic beyond $\lambda \sim 0.12$ for the case of $x=0.5$ as shown in figure 17(a). However for larger values of $\lambda$, two loop corrections overtake the one loop corrections and that is why we get the upper band of allowed values of $\lambda$. Correct Higgs mass is achievable for $\lambda \sim 2.0$ (see figure 17(b)). For $x=0.1$, there is only one band of parameter space allowed for $\lambda$ and maximum allowed value of $\lambda$ is $\sim 1.8$ as shown in figure 17(c). We have a parameter space near $\lambda \gtrsim 1.5$ and $y_{N} \gtrsim 0.2$ where we can achieve $125 \mathrm{GeV}$ Higgs mass (figure 17(d)).

Four benchmark points for this model are shown in table 18. In the second benchmark point, $M_{\tilde{L}}^{2}-m_{R}^{2}$ is slightly large, so the contribution to the Higgs mass is less as compare to other benchmark points. 


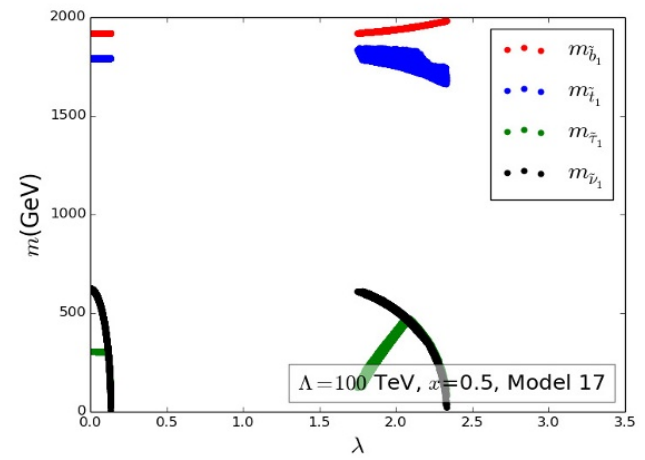

(a)

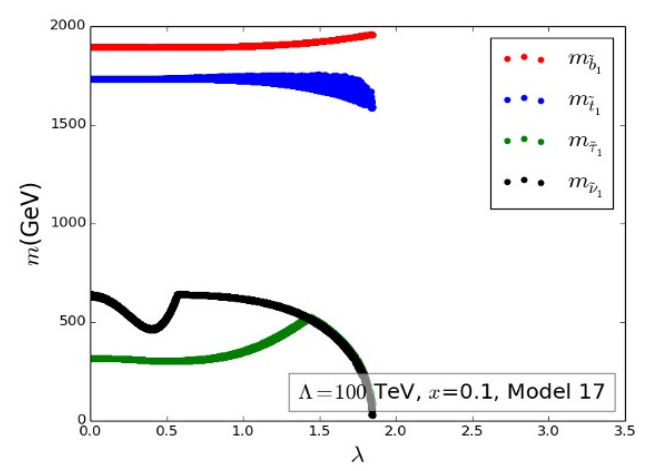

(c)

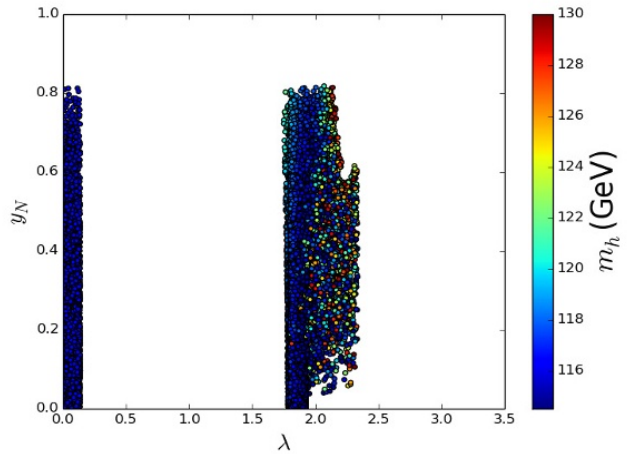

(b)

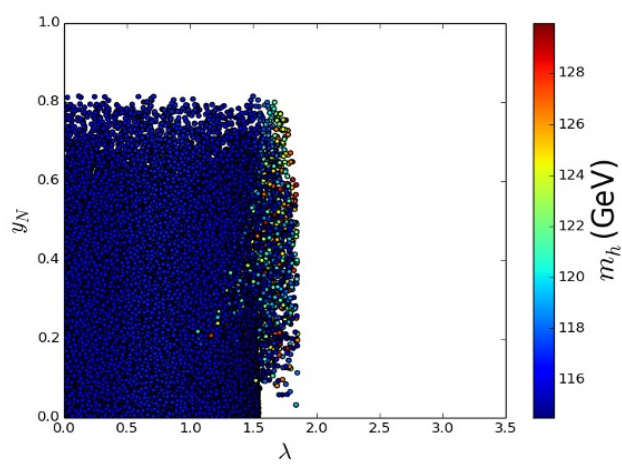

(d)

Figure 17. Model 17: spectrum variation with $\lambda$ and Higgs mass data points in $y_{N}$ and $\lambda$ plane. Please see caption of figure 1 for details of notation.

\section{Discussion and conclusions}

Gauge mediated supersymmetry breaking models are interesting due to their flavour blindness and unique phenomenological features like gravitino LSP etc. The discovery of the Higgs particle and the non-discovery of the coloured supersymmetric partners at the LHC has however put strong constraints on Gauge Mediated Supersymmetry Breaking models with vanishing A-terms, i.e, both minimal and general forms of them.

Allowing for direct matter-messenger interactions in addition to the standard gauge interactions is being pursued as one of the interesting solutions which allows for a light spectrum $\sim 1 \mathrm{TeV}$ and a light $\mathrm{CP}$ even Higgs boson at $125 \mathrm{GeV}$. Several studies have been presented using these ideas, which culminated in survey refs. [1, 2]. In the present work, we extended the survey to a particular extension of MSSM including right handed neutrinos. We chose the inverse seesaw model because of the possible large impact on the corrections to the Higgs mass. However with the usual boundary conditions prescribed for minimal gauge mediation it is hard to generate a large enhancement to the Higgs mass through the neutrino/sneutrino sector. In general gauge mediation framework, a large separate parameter for the sleptons should be prescribed such that these corrections 


\begin{tabular}{|c|c|c|c|c|c|c|c|c|c|}
\hline Parameter & $\mathrm{x}=0.5$ & $\mathrm{x}=0.5$ & $\mathrm{x}=0.1$ & $\mathrm{x}=0.1$ & Parameter & $\mathrm{x}=0.5$ & $\mathrm{x}=0.5$ & $\mathrm{x}=0.1$ & $\mathrm{x}=0.1$ \\
\hline$\lambda$ & 2.02 & 1.81 & 1.43 & 1.02 & $y_{N}$ & 0.23 & 0.28 & 0.39 & 0.18 \\
\hline$A_{N}$ & -7680.1 & -6180.1 & -3781.2 & -1984.2 & $A_{t}$ & -569.7 & -562.9 & -667.4 & -697.2 \\
$m_{R}$ & 5380.8 & 3372.8 & 5507.2 & 2412.2 & $\mu$ & 869. & 918. & 1086. & 762. \\
$m_{\tilde{\nu}_{1}}$ & 5663. & 2752. & 5054. & 2371. & $m_{\tilde{\nu}_{2,3}} / m_{\nu_{2,3}}$ & 5378. & 3377. & 5509. & 2419. \\
\hline$m_{h}$ & 124.50 & 119.16 & 124.46 & 124.61 & $m_{H}$ & 957. & 1067. & 1164. & 976. \\
$m_{h}^{0+\Delta}$ & 111.62 & 116.60 & 114.22 & 116.17 & $m_{A^{0}}$ & 983. & 1080. & 1200. & 978. \\
$m_{h}^{0}$ & 116.23 & 116.23 & 116.33 & 116.33 & $m_{H^{ \pm}}$ & 974. & 1079. & 1200. & 981. \\
\hline$M_{S}$ & 1806. & 1837. & 1762. & 1786. & $m_{\tilde{g}}$ & 2188. & 2188. & 2112. & 2111. \\
$\tilde{t}_{1}$ & 1754. & 1788. & 1700. & 1723. & $\tilde{t}_{2}$ & 1860. & 1888. & 1826. & 1852. \\
$\tilde{b}_{1}$ & 1939. & 1922. & 1916. & 1898. & $\tilde{b}_{2}$ & 1944. & 1927. & 1923. & 1904. \\
$\tilde{\tau}_{1}$ & 399. & 180. & 523. & 349. & $\tilde{\tau}_{2}$ & 5659. & 2758. & 5058. & 2388. \\
$\tilde{u}_{1}$ & 1885. & 1918. & 1858. & 1897. & $\tilde{u}_{2}$ & 1979. & 1972. & 1954. & 1942. \\
$\tilde{d}_{1}$ & 1944. & 1927. & 1923. & 1904. & $\tilde{d}_{2}$ & 1969. & 1960. & 1944. & 1926. \\
$\tilde{e}_{1}$ & 517. & 393. & 524. & 397. & $\tilde{e}_{2}$ & 589. & 603. & 614. & 621. \\
$N_{1}$ & 430. & 431. & 412. & 411. & $N_{2}$ & 788. & 799. & 783. & 719. \\
$N_{3}$ & 875. & 924. & 1093. & 768. & $N_{4}$ & 919. & 955. & 1105. & 845. \\
$C_{1}$ & 773. & 785. & 765. & 709. & $C_{2}$ & 906. & 944. & 1095. & 830. \\
\hline
\end{tabular}

Table 18. Benchmark points for Model 17. See caption of table 2 for details of notation.

become significant. Another way to increase the neutrino/sneutrino contributions would be to generate a large sneutrino mixing parameter through matter-messenger mixing. In the present work, we explored this possibility in great detail and surveyed all possible matter messenger mixing terms with leptonic doublet fields (L) and right handed neutrino fields $\left(N^{c}\right)$. It turns out that in almost all the successful models, in addition to $X_{N}$, a significant correction to $M_{\tilde{L}}^{2}$ is also generated.

We classified all possible models by imposing lepton number on the messenger sector fields also. We found 17 such models. Both of the Type I (matter-messenger-messenger) and Type II (matter-matter-messenger). We analysed each model in detail by doing a full numerical analysis. The results are summarized in the table 19.

We perform thorough numerical analysis by using SuSeFLAV considering $\tan \beta=10$, $\mu_{s}=10^{-4} \times m_{e}$ and $\Lambda=100 \mathrm{TeV}$ (see eq. (4.1)) for $x=0.1$ and 0.5. The parameter $x$ played an important role and the competition between one-loop and two-loop messengermatter corrections is clear from the plots for these two values of $x$. In model $2,3,4,5,7$, $11,12,13,14,15$ and 17, there are two bands in $\lambda$, for $x=0.5$. Except for the model 11 and 12 , these bands get merged in to one for $x=0.1$.

In the models $1,2,3,5,6,8,9$, and 10, we do not get correct Higgs mass (see table 19) because correction to $M_{\tilde{L}}^{2}$ is either zero or negative in the boundary. In the rest of the models, $L$ directly couples to the messenger fields and thus in these model, $M_{\tilde{L}}^{2}$ gets both 


\begin{tabular}{|c|c|c|c|c|c|}
\hline Model & Interaction & $125 \mathrm{GeV}$ Higgs & \multicolumn{2}{c|}{ Range of $\lambda$} \\
\hline \multicolumn{5}{|c|}{ Models with $N^{c}$} \\
\hline \multicolumn{7}{|c|}{} & \multicolumn{1}{|c|}{$\mathrm{x}=0.5$} & $\mathrm{x}=0.1$ & $\mathrm{x}=0.5$ & $\mathrm{x}=0.1$ \\
\hline 1. & $N^{c} Q \bar{Q}_{m}$ & $\times$ & $\times$ & 1.48 & 1.24 \\
\hline 2. & $N^{c} U^{c} \bar{U}_{m}^{c}$ & $\times$ & $\times$ & 1.86 & 1.60 \\
\hline 3. & $N^{c} D^{c} \bar{D}_{m}^{c}$ & $\times$ & $\times$ & 1.15 & 0.94 \\
\hline 4. & $N^{c} L H_{u}^{m}$ & $\checkmark$ & $\checkmark$ & 1.84 & 1.61 \\
\hline 5. & $N^{c} E^{c} \bar{E}_{m}^{c}$ & $\times$ & $\times$ & 2.10 & 1.60 \\
\hline 6. & $N^{c} H_{u} H_{d}^{m}$ & $\times$ & $\times$ & 1.0 & 0.57 \\
\hline 7. & $N^{c} H_{d} H_{u}^{m}$ & $\checkmark$ & $\checkmark$ & 1.68 & 1.52 \\
\hline 8. & $\frac{1}{2}\left(N^{c}\right)^{2} S_{m}$ & $\times$ & $\times$ & $\sqrt{4 \pi}$ & $\sqrt{4 \pi}$ \\
\hline 9. & $N^{c} S S_{m}$ & $\times$ & $\times$ & $1.05-\sqrt{4 \pi}$ & $0.02-\sqrt{4 \pi}$ \\
\hline 10. & $N^{c} H_{u}^{m} H_{d}^{m}$ & $\times$ & $\times$ & $\sqrt{4 \pi}$ & $\sqrt{4 \pi}$ \\
\hline \multicolumn{7}{|c|}{ Models with L } \\
\hline 11. & $L Q \bar{D}_{m}^{c}$ & $\checkmark$ & $\checkmark$ & 1.21 & 1.09 \\
\hline 12. & $L D^{c} \bar{Q}_{m}$ & $\checkmark$ & $\checkmark$ & 1.21 & 2.23 \\
\hline 13. & $L E^{c} H_{d}^{m}$ & $\checkmark$ & $\checkmark$ & 1.20 & 0.93 \\
\hline 14. & $L H_{d} E_{m}^{c}$ & $\checkmark$ & $\checkmark$ & 2.63 & 2.26 \\
\hline 15. & $L H_{u} S_{m}$ & $\checkmark$ & $\times$ & 1.13 & 0.62 \\
\hline 16. & $L S H_{u}^{m}$ & $\checkmark$ & $\checkmark$ & $0.98-1.83$ & $0.34-1.61$ \\
\hline 17. & $L H_{u}^{m} S_{m}$ & $\checkmark$ & $\checkmark$ & 2.34 & 1.85 \\
\hline
\end{tabular}

Table 19. Summary of all the models for both $x=0.5$ and $x=0.1$ case. The symbol $\checkmark$ shows that a $125 \mathrm{GeV}$ Higgs can be achieved otherwise the symbol $\times$ is used. In the last two columns the range of the $\lambda$ is shown. We mention the upper allowed value of $\lambda$ and for the models where there is a lower bound we mention the allowed range (see model 9 and 16).

the positive two loop and negative one loop corrections at the boundary. For $x=0.1$, two loop effect dominates over the one loop effect and $M_{\tilde{L}}^{2}$ becomes comparable to $m_{R}$. As a consequence, Higgs mass gets significant correction.

EWSB also played an important role in several models where $H_{u}$ and $H_{d}$ field are present in the messenger-matter Interaction. For example, in models 14 , for $x=0.5, M_{H_{u}}$ gets both the negative and positive corrections whereas for $x=0.1$ positive contributions dominate over the negative one-loop contribution. Thus with $\lambda$, this mass increases and EWSB can not be achieved beyond some value. 
Models with messenger-matter corrections to $M_{\tilde{S}}$ are also interesting. The requirement of non-tachyonic $M_{\tilde{S}}$ puts lower bound on value of $\lambda$. In model 16 , for $x=0.5$, complete parameter space is ruled out by this condition.

In the models where $Q, U^{c}$ and $H_{u}$ are interacting with the messenger field $A_{t}$ term is generated at the boundary and these models are 1,2, 6, 11 and 15. However, in models 1, 2 and 6 one cannot achieve a $125 \mathrm{GeV}$ Higgs even for $x=0.1$. Among all the 17 models, model 11 has benchmark points with the lightest $m_{\tilde{t}_{1}}$ mass. Thus this is the most promising model in the context of fine-tuning.

The set of models presented here has several unique features. The mass spectra is like the minimal Gauge mediation models in the colored sector; the stops are light with small or negligible mixing. In the leptonic sector, the charged sleptons are light (typically except one eigenvalue), whereas the sneutrinos are heavy, close to the right handed neutrino mass scale. The typical collider signatures would mimic that of a minimal GMSB model, with large stau mixing, spectrum in LHC accessible range, and still have the lightest CP even higgs mass at $125 \mathrm{GeV}$.

\section{Acknowledgments}

We thank Biplob Bhattacherjee and Eung Jin Chun for discussions. SKV thanks CPHT, Ecole Polytechnique for hospitality during the final stages of this work. We thank Carlos E. M. Wagner for raising questions regarding factor of $1 / 2$ in the Higgs mass calculation.

\section{A One loop neutrino-sneutrino corrections to the Higgs mass}

Tree level scalar potential $\left(V_{0}\right)$ gets modified by one-loop Coleman-Weinberg potential $(\triangle V)[69]$. In principle one has to calculate vacuum by minimizing the complete potential i.e. $V_{0}+\triangle V$; however in practice $\mathrm{VEV}$ of the Higgs fields are calculated by minimizing the tree level potential. ${ }^{1}$ Thus the Higgs mass, which should be double derivative, contains some extra single derivative terms:

$$
\Delta M_{i j}^{2}=\frac{1}{2}\left(\frac{\partial^{2} \Delta V}{\partial H_{i} \partial H_{j}}-\frac{\delta_{i j}}{H_{i}} \frac{\partial \Delta V}{\partial H_{i}}\right),
$$

where $i, j=u, d$. Corrections from the top-stop sector are well-known. Here we are reviewing the correction to the Higgs mass from neutrino-sneutrino sector. Mass eigenvalues of these fields are already mentioned in section 3. Plugging these values into ColemanWeinberg potential, $\Delta M_{\nu i j}^{2}$ has to be calculated. We drop suffix $\nu$ from these terms for brevity.

$$
\begin{aligned}
\Delta M_{\mathrm{uu}}^{2}= & \left.\frac{1}{32 \pi^{2}} \sum_{\alpha=1,2,3}\left(\tilde{L}_{\alpha} \tilde{B}_{\alpha u}^{2}+m_{\tilde{\nu}_{\alpha}}^{2}\left(\tilde{A}_{\alpha u u}-\frac{\tilde{B}_{\alpha u}}{H_{u}}\right)\left(\tilde{L}_{\alpha}-1\right)\right)\right|_{H_{u}=\left\langle H_{u}\right\rangle} \\
& -\left.\frac{1}{32 \pi^{2}} \sum_{\alpha=1,2,3}\left(L_{\alpha} B_{\alpha u}^{2}+m_{\nu_{\alpha}}^{2}\left(A_{\alpha u u}-\frac{B_{\alpha u}}{H_{u}}\right)\left(L_{\alpha}-1\right)\right)\right|_{H_{u}=\left\langle H_{u}\right\rangle},
\end{aligned}
$$

\footnotetext{
${ }^{1}$ There is a conflict of factor of $1 / 2$ in eq. (A.1) between refs. [49] and [51]. We resolve this issue and agree with [51].
} 


$$
\begin{aligned}
& \Delta M_{\mathrm{dd}}^{2}=\left.\frac{1}{32 \pi^{2}} \sum_{\alpha=1,2,3}\left(\tilde{L}_{\alpha} \tilde{B}_{\alpha d}^{2}+m_{\tilde{\nu}_{\alpha}}^{2}\left(\tilde{A}_{\alpha d d}-\frac{\tilde{B}_{\alpha d}}{H_{d}}\right)\left(\tilde{L}_{\alpha}-1\right)\right)\right|_{H_{d}=\left\langle H_{d}\right\rangle}, \\
& \Delta M_{\mathrm{ud}}^{2}=\left.\frac{1}{32 \pi^{2}} \sum_{\alpha=1,2,3}\left(\tilde{L}_{\alpha} \tilde{B}_{\alpha u} \tilde{B}_{\alpha d}+m_{\tilde{\nu}_{\alpha}}^{2} \tilde{A}_{\alpha u d}\left(\tilde{L}_{\alpha}-1\right)\right)\right|_{H_{u}=\left\langle H_{u}\right\rangle, H_{d}=\left\langle H_{d}\right\rangle},
\end{aligned}
$$

where

$$
\begin{aligned}
L_{\alpha} & =\log \left(\frac{m_{\nu_{\alpha}}^{2}}{M_{\mathrm{SUSY}}^{2}}\right), & \tilde{L}_{\alpha} & =\log \left(\frac{m_{\tilde{\nu}_{\alpha}}^{2}}{M_{\mathrm{SUSY}}^{2}}\right), \\
B_{\alpha j} & =\frac{\partial m_{\nu_{\alpha}}^{2}}{\partial H_{j}}, & \tilde{B}_{\alpha j} & =\frac{\partial m_{\tilde{\nu}_{\alpha}}^{2}}{\partial H_{j}}, \\
A_{\alpha j k} & =\frac{\partial B_{\alpha j}}{\partial H_{k}}, & \tilde{A}_{\alpha j k} & =\frac{\partial B_{\alpha j}}{\partial H_{k}} .
\end{aligned}
$$

Explicit expressions for $\tilde{B}_{\alpha i}$ are as follows:

$$
\begin{aligned}
\tilde{B}_{1 u} & =\frac{2 v_{d} \mu X_{N} y_{N}^{2}}{d_{1}}+2 v_{u}\left(1+\frac{m_{R}^{2}}{d_{2}}+\frac{X_{N}^{2}}{d_{1}}\right) y_{N}^{2}, \\
\tilde{B}_{1 d} & =\frac{-2 v_{u} \mu X_{N} y_{N}^{2}}{d_{1}} \\
\tilde{B}_{2 u} & =\frac{-2 v_{d} \mu X_{N} y_{N}^{2}}{d_{1}}+2 v_{u}\left(1-\frac{X_{N}^{2}}{d_{1}}\right) y_{N}^{2}, \\
\tilde{B}_{2 d} & =\frac{2 v_{u} \mu X_{N} y_{N}^{2}}{d_{1}} \\
\tilde{B}_{3 u} & =\frac{-2 v_{u} m_{R}^{2} y_{N}^{2}}{d_{2}} \\
\tilde{B}_{3 d} & =0 .
\end{aligned}
$$

Similarly one can calculate the $\tilde{A}_{\alpha i j}$ terms:

$$
\begin{aligned}
& \tilde{A}_{1 u u}=\frac{2 v_{d}^{2} \mu^{2} y_{N}^{2}}{v_{u}^{2} d_{1}}+\frac{4 v_{d} \mu X_{N} y_{N}^{2}}{v_{u} d_{1}}+2\left(1+\frac{m_{R}^{2}}{d_{2}}+\frac{X_{N}^{2}}{d_{1}}\right) y_{N}^{2}, \\
& \tilde{A}_{1 u d}=\frac{-2 v_{d} \mu^{2} y_{N}^{2}}{v_{u} d_{1}}-\frac{2 \mu X_{N} y_{N}^{2}}{d_{1}}, \\
& \tilde{A}_{1 d d}=\frac{2 \mu^{2} y_{N}^{2}}{d_{1}} \\
& \tilde{A}_{2 u u}=\frac{-2 v_{d}^{2} \mu^{2} y_{N}^{2}}{v_{u}^{2} d_{1}}-\frac{4 v_{d} \mu X_{N} y_{N}^{2}}{v_{u} d_{1}}+2\left(1-\frac{X_{N}^{2}}{d_{1}}\right) y_{N}^{2}, \\
& \tilde{A}_{2 u d}=\frac{2 v_{d} \mu^{2} y_{N}^{2}}{v_{u} d_{1}}+\frac{2 \mu X_{N} y_{N}^{2}}{d_{1}}, \\
& \tilde{A}_{2 d d}=\frac{-2 \mu^{2} y_{N}^{2}}{d_{1}} \\
& \tilde{A}_{3 u u}=\frac{-2 m_{R}^{2} y_{N}^{2}}{d_{2}}
\end{aligned}
$$




$$
\begin{aligned}
\tilde{A}_{3 u d} & =0, \\
\tilde{A}_{3 d d} & =0 .
\end{aligned}
$$

Similarly $B_{\alpha i}$ terms are:

$$
\begin{aligned}
B_{1 u} & =\frac{4 v_{u}^{3} y_{N}^{4} \mu_{S}^{2}}{m_{R}^{4}} \\
B_{2 u} & =\frac{2 v_{u} y_{N}^{2}\left(\frac{v_{u}^{2} y_{N}^{2}}{2 m_{R}}+m_{R}\right)}{m_{R}} \\
B_{3 u} & =\frac{2 v_{u} y_{N}^{2}\left(\frac{v_{u}^{2} y_{N}^{2}}{2 m_{R}}+m_{R}\right)}{m_{R}} \\
B_{1 d} & =0 \\
B_{2 d} & =0 \\
B_{3 d} & =0
\end{aligned}
$$

Finally the $A_{\alpha i j}$ terms are as follows:

$$
\begin{aligned}
A_{1 u u} & =\frac{12 v_{u}^{2} y_{N}^{4} \mu_{S}^{2}}{m_{R}^{4}} \\
A_{2 u u} & =\frac{2 v_{u}^{2} y_{N}^{4}}{m_{R}^{2}}+\frac{2 y_{N}^{2}\left(\frac{v_{u}^{2} y_{N}^{2}}{2 m_{R}}+m_{R}\right)}{m_{R}} \\
A_{3 u u} & =\frac{2 v_{u}^{2} y_{N}^{4}}{m_{R}^{2}}+\frac{2\left(\frac{v_{u}^{2} y_{N}^{2}}{2 m_{R}}+m_{R}\right) y_{N}^{2}}{m_{R}} \\
A_{1 u d} & =0 \\
A_{2 u d} & =0 \\
A_{3 u d} & =0 \\
A_{1 d d} & =0 \\
A_{2 d d} & =0 \\
A_{3 d d} & =0
\end{aligned}
$$

Now being equipped with the above formulas one can calculate Higgs mass. We are here giving a simple formula for alignment limit:

$$
m_{h}^{2}=m_{Z}^{2} \cos ^{2} 2 \beta+\text { top-stop correction }+\sin ^{2} \beta \triangle M_{u u}^{2}+\sin 2 \beta \triangle M_{u d}^{2}+\cos ^{2} \beta \triangle M_{d d}^{2} .
$$

Open Access. This article is distributed under the terms of the Creative Commons Attribution License (CC-BY 4.0), which permits any use, distribution and reproduction in any medium, provided the original author(s) and source are credited.

\section{References}

[1] P. Byakti and T.S. Ray, Burgeoning the Higgs mass to 125 GeV through messenger-matter interactions in GMSB models, JHEP 05 (2013) 055 [arXiv:1301.7605] [INSPIRE]. 
[2] J.A. Evans and D. Shih, Surveying extended GMSB models with $m_{h}=125 \mathrm{GeV}$, JHEP 08 (2013) 093 [arXiv: 1303.0228] [INSPIRE].

[3] A. Salam and J.A. Strathdee, Supergauge transformations, Nucl. Phys. B 76 (1974) 477 [INSPIRE].

[4] P. Fayet and S. Ferrara, Supersymmetry, Phys. Rept. 32 (1977) 249 [INSPIRE].

[5] H.P. Nilles, Supersymmetry, supergravity and particle physics, Phys. Rept. 110 (1984) 1 [INSPIRE].

[6] J. Wess and J. Bagger, Supersymmetry and supergravity, Princeton Univ. Pr., Princeton U.S.A., (1992) [INSPIRE].

[7] S.P. Martin, A supersymmetry primer, Adv. Ser. Direct. High Energy Phys. 18 (1998) 1 [Adv. Ser. Direct. High Energy Phys. 21 (2010) 1] [hep-ph/9709356] [INSPIRE].

[8] P. Fayet, Supersymmetry and weak, electromagnetic and strong interactions, Phys. Lett. B 64 (1976) 159 [INSPIRE].

[9] S.K. Vempati, Introduction to MSSM, arXiv:1201.0334 [INSPIRE].

[10] G.F. Giudice and R. Rattazzi, Theories with gauge mediated supersymmetry breaking, Phys. Rept. 322 (1999) 419 [hep-ph/9801271] [INSPIRE].

[11] ATLAS collaboration, Observation of a new particle in the search for the Standard Model Higgs boson with the ATLAS detector at the LHC, Phys. Lett. B 716 (2012) 1 [arXiv: 1207.7214] [INSPIRE].

[12] CMS collaboration, Observation of a new boson at a mass of $125 \mathrm{GeV}$ with the CMS experiment at the LHC, Phys. Lett. B 716 (2012) 30 [arXiv:1207.7235] [INSPIRE].

[13] P. Draper, P. Meade, M. Reece and D. Shih, Implications of a $125 \mathrm{GeV}$ Higgs for the MSSM and low-scale SUSY breaking, Phys. Rev. D 85 (2012) 095007 [arXiv:1112.3068] [INSPIRE].

[14] K. Intriligator and M. Sudano, General gauge mediation with gauge messengers, JHEP 06 (2010) 047 [arXiv: 1001.5443] [INSPIRE].

[15] P. Grajek, A. Mariotti and D. Redigolo, Phenomenology of general gauge mediation in light of a $125 \mathrm{GeV}$ Higgs, JHEP 07 (2013) 109 [arXiv: 1303.0870] [INSPIRE].

[16] Z. Kang, T. Li, T. Liu, C. Tong and J.M. Yang, A heavy SM-like Higgs and a light stop from Yukawa-deflected gauge mediation, Phys. Rev. D 86 (2012) 095020 [arXiv:1203.2336] [INSPIRE].

[17] A. Albaid and K.S. Babu, Higgs boson of mass $125 \mathrm{GeV}$ in GMSB models with messenger-matter mixing, Phys. Rev. D 88 (2013) 055007 [arXiv:1207.1014] [INSPIRE].

[18] T. Han and R.-J. Zhang, Direct messenger-matter interactions in gauge-mediated supersymmetry breaking models, Phys. Lett. B 428 (1998) 120 [hep-ph/9802422] [INSPIRE].

[19] N. Craig, S. Knapen, D. Shih and Y. Zhao, A complete model of low-scale gauge mediation, JHEP 03 (2013) 154 [arXiv:1206.4086] [INSPIRE].

[20] J.L. Evans, M. Ibe and T.T. Yanagida, Relatively heavy Higgs boson in more generic gauge mediation, Phys. Lett. B 705 (2011) 342 [arXiv:1107.3006] [INSPIRE].

[21] S.P. Martin and J.D. Wells, Implications of gauge-mediated supersymmetry breaking with vector-like quarks and $a \sim 125 \mathrm{GeV}$ Higgs boson, Phys. Rev. D 86 (2012) 035017 [arXiv:1206.2956] [INSPIRE]. 
[22] J.A. Casas, J.M. Moreno, S. Robles and K. Rolbiecki, Reducing the fine-tuning of gauge-mediated SUSY breaking, Eur. Phys. J. C 76 (2016) 450 [arXiv:1602.06892] [INSPIRE].

[23] R. Ding, T. Li, L. Wang and B. Zhu, Towards the natural gauge mediation, JHEP 10 (2015) 154 [arXiv: 1506.00359] [inSPIRE].

[24] A. Delgado, M. Garcia-Pepin and M. Quirós, GMSB with light stops, JHEP 08 (2015) 159 [arXiv: 1505.07469] [INSPIRE].

[25] T. Jeliński, SO(10) inspired extended GMSB models, PoS (CORFU2014) 089 [arXiv: 1505.06722] [INSPIRE].

[26] J.A. Evans, D. Shih and A. Thalapillil, Chiral flavor violation from extended gauge mediation, JHEP 07 (2015) 040 [arXiv: 1504.00930] [INSPIRE].

[27] M. Backović, A. Mariotti and M. Spannowsky, Signs of tops from highly mixed stops, JHEP 06 (2015) 122 [arXiv: 1504.00927] [INSPIRE].

[28] A. Basirnia, D. Egana-Ugrinovic, S. Knapen and D. Shih, $125 \mathrm{GeV}$ Higgs from tree-level A-terms, JHEP 06 (2015) 144 [arXiv:1501.00997] [INSPIRE].

[29] W. Fischler and W. Tangarife, Vector-like fields, messenger mixing and the Higgs mass in gauge mediation, JHEP 05 (2014) 151 [arXiv:1310.6369] [INSPIRE].

[30] T. Jeliński, On messengers couplings in extended GMSB models, JHEP 09 (2013) 107 [arXiv: 1305.6277] [INSPIRE].

[31] L. Calibbi, P. Paradisi and R. Ziegler, Gauge mediation beyond minimal flavor violation, JHEP 06 (2013) 052 [arXiv: 1304.1453] [INSPIRE].

[32] V.S. Mummidi and S.K. Vempati, A little more gauge mediation and the light Higgs mass, Nucl. Phys. B 881 (2014) 181 [arXiv:1311.4280] [INSPIRE].

[33] K.S. Babu, I. Gogoladze, M.U. Rehman and Q. Shafi, Higgs boson mass, sparticle spectrum and little hierarchy problem in extended MSSM, Phys. Rev. D 78 (2008) 055017 [arXiv:0807.3055] [INSPIRE].

[34] M. Endo, K. Hamaguchi, S. Iwamoto and N. Yokozaki, Higgs mass and muon anomalous magnetic moment in supersymmetric models with vector-like matters, Phys. Rev. D 84 (2011) 075017 [arXiv: 1108.3071] [INSPIRE].

[35] M. Endo, K. Hamaguchi, S. Iwamoto and N. Yokozaki, Higgs mass, muon g-2 and LHC prospects in gauge mediation models with vector-like matters, Phys. Rev. D 85 (2012) 095012 [arXiv: 1112.5653] [INSPIRE].

[36] K. Nakayama and N. Yokozaki, Peccei-Quinn extended gauge-mediation model with vector-like matter, JHEP 11 (2012) 158 [arXiv:1204.5420] [INSPIRE].

[37] M. Asano, Y. Nakai and N. Yokozaki, A new scheme for NMSSM in gauge mediation, JHEP 01 (2016) 066 [arXiv:1510.08033] [INSPIRE].

[38] G. Mittag, Search for a light NMSSM Higgs boson in the b-quark final state at the LHC, Ph.D. thesis, DESY-THESIS-2015-029, Dept. Phys., U. Hamburg, Hamburg Germany, (2015) [INSPIRE].

[39] K. Kowalska, J. Pawełczyk and E.M. Sessolo, Flavored gauge mediation in the Peccei-Quinn NMSSM, JHEP 12 (2015) 148 [arXiv:1508.04142] [INSPIRE]. 
[40] F. Domingo, A new tool for the study of the CP-violating NMSSM, JHEP 06 (2015) 052 [arXiv: 1503.07087] [INSPIRE].

[41] M. Cahill-Rowley, J. Gainer, J. Hewett and T. Rizzo, Towards a supersymmetric description of the Fermi galactic center excess, JHEP 02 (2015) 057 [arXiv:1409.1573] [INSPIRE].

[42] U. Ellwanger and A.M. Teixeira, NMSSM with a singlino LSP: possible challenges for searches for supersymmetry at the LHC, JHEP 10 (2014) 113 [arXiv:1406.7221] [INSPIRE].

[43] P. Athron, J.-H. Park, D. Stöckinger and A. Voigt, FlexibleSUSY - a spectrum generator generator for supersymmetric models, Comput. Phys. Commun. 190 (2015) 139 [arXiv: 1406.2319] [INSPIRE].

[44] B.C. Allanach, P. Athron, L.C. Tunstall, A. Voigt and A.G. Williams, Next-to-minimal SOFTSUSY, Comput. Phys. Commun. 185 (2014) 2322 [arXiv:1311.7659] [InSPIRE].

[45] T.T. Yanagida, N. Yokozaki and K. Yonekura, Higgs boson mass in low scale gauge mediation models, JHEP 10 (2012) 017 [arXiv:1206.6589] [INSPIRE].

[46] A. de La Puente and W. Tangarife, A singlet extension of the MSSM with a dark matter portal, JHEP 07 (2014) 087 [arXiv: 1309.6359] [INSPIRE].

[47] M.E. Krauss, W. Porod and F. Staub, $\mathrm{SO}(10)$ inspired gauge-mediated supersymmetry breaking, Phys. Rev. D 88 (2013) 015014 [arXiv:1304.0769] [INSPIRE].

[48] A.M. Iyer, V.S. Mummidi and S.K. Vempati, Gravitational rescue of minimal gauge mediation, Int. J. Mod. Phys. A 31 (2016) 1650045 [arXiv:1408.4462] [inSPIRE].

[49] A. Elsayed, S. Khalil and S. Moretti, Higgs mass corrections in the SUSY B-L model with inverse seesaw, Phys. Lett. B 715 (2012) 208 [arXiv:1106.2130] [INSPIRE].

[50] P. Draper and H.E. Haber, Decoupling of the right-handed neutrino contribution to the Higgs mass in supersymmetric models, Eur. Phys. J. C 73 (2013) 2522 [arXiv:1304.6103] [INSPIRE].

[51] J. Guo, Z. Kang, T. Li and Y. Liu, Higgs boson mass and complex sneutrino dark matter in the supersymmetric inverse seesaw models, JHEP 02 (2014) 080 [arXiv:1311.3497] [INSPIRE].

[52] E.J. Chun, V.S. Mummidi and S.K. Vempati, Anatomy of Higgs mass in supersymmetric inverse seesaw models, Phys. Lett. B 736 (2014) 470 [arXiv:1405.5478] [INSPIRE].

[53] L. Calibbi, L. Ferretti, A. Romanino and R. Ziegler, Gauge coupling unification, the GUT scale and magic fields, Phys. Lett. B 672 (2009) 152 [arXiv:0812.0342] [INSPIRE].

[54] P. Byakti and D. Ghosh, Magic messengers in gauge mediation and signal for $125 \mathrm{GeV}$ boosted Higgs boson, Phys. Rev. D 86 (2012) 095027 [arXiv: 1204.0415] [INSPIRE].

[55] P. Meade, N. Seiberg and D. Shih, General gauge mediation, Prog. Theor. Phys. Suppl. 177 (2009) 143 [arXiv:0801.3278] [INSPIRE].

[56] T.T. Dumitrescu, Z. Komargodski, N. Seiberg and D. Shih, General messenger gauge mediation, JHEP 05 (2010) 096 [arXiv: 1003.2661] [INSPIRE].

[57] M. Dine, Y. Nir and Y. Shirman, Variations on minimal gauge mediated supersymmetry breaking, Phys. Rev. D 55 (1997) 1501 [hep-ph/9607397] [INSPIRE].

[58] G.R. Dvali, G.F. Giudice and A. Pomarol, The $\mu$ problem in theories with gauge mediated supersymmetry breaking, Nucl. Phys. B 478 (1996) 31 [hep-ph/9603238] [INSPIRE]. 
[59] A. Delgado, G.F. Giudice and P. Slavich, Dynamical $\mu$ term in gauge mediation, Phys. Lett. B 653 (2007) 424 [arXiv:0706.3873] [INSPIRE].

[60] Z. Komargodski and N. Seiberg, $\mu$ and general gauge mediation, JHEP 03 (2009) 072 [arXiv:0812.3900] [INSPIRE].

[61] Z. Chacko and E. Ponton, Yukawa deflected gauge mediation, Phys. Rev. D 66 (2002) 095004 [hep-ph/0112190] [INSPIRE].

[62] G.F. Giudice and R. Rattazzi, Extracting supersymmetry breaking effects from wave function renormalization, Nucl. Phys. B 511 (1998) 25 [hep-ph/9706540] [INSPIRE].

[63] A. Mariotti, M. Backovic and M. Spannowsky, Signs of tops from highly mixed stops, PoS (PLANCK 2015) 080 [INSPIRE].

[64] L. Calibbi, P. Paradisi and R. Ziegler, Lepton flavor violation in flavored gauge mediation, Eur. Phys. J. C 74 (2014) 3211 [arXiv: 1408.0754] [INSPIRE].

[65] R.N. Mohapatra and P.B. Pal, Massive neutrinos in physics and astrophysics. Second edition, World Sci. Lect. Notes Phys. 60 (1998) 1 [World Sci. Lect. Notes Phys. 72 (2004) 1] [INSPIRE].

[66] R.N. Mohapatra and J.W.F. Valle, Neutrino mass and baryon number nonconservation in superstring models, Phys. Rev. D 34 (1986) 1642 [INSPIRE].

[67] I. Gogoladze, B. He and Q. Shafi, Inverse seesaw in NMSSM and 126 GeV Higgs boson, Phys. Lett. B 718 (2013) 1008 [arXiv:1209.5984] [INSPIRE].

[68] F. del Aguila, J. de Blas and M. Pérez-Victoria, Effects of new leptons in electroweak precision data, Phys. Rev. D 78 (2008) 013010 [arXiv: 0803.4008] [INSPIRE].

[69] S.R. Coleman and E.J. Weinberg, Radiative corrections as the origin of spontaneous symmetry breaking, Phys. Rev. D 7 (1973) 1888 [InSPIRE].

[70] CMS collaboration, Search for supersymmetry in events with photons and missing transverse energy in pp collisions at $13 \mathrm{TeV}$, submitted to Phys. Lett. B (2016) [arXiv:1611.06604] [INSPIRE].

[71] ATLAS collaboration, Search for photonic signatures of gauge-mediated supersymmetry in 8 TeV pp collisions with the ATLAS detector, Phys. Rev. D 92 (2015) 072001 [arXiv: 1507.05493] [INSPIRE].

[72] M. Viel, J. Lesgourgues, M.G. Haehnelt, S. Matarrese and A. Riotto, Constraining warm dark matter candidates including sterile neutrinos and light gravitinos with WMAP and the

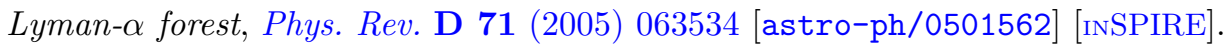

[73] M. Kawasaki, F. Takahashi and T.T. Yanagida, Gravitino overproduction in inflaton decay, Phys. Lett. B 638 (2006) 8 [hep-ph/0603265] [INSPIRE].

[74] D. Chowdhury, R. Garani and S.K. Vempati, SuSeFLAV: a program for calculating supersymmetric spectra and lepton flavor violation, Pramana 79 (2012) 887 [arXiv: 1112.4403] [INSPIRE]. 